\title{
Polycyclic Aromatic Hydrocarbon in Protoplanetary Disks around Herbig Ae/Be and T Tauri Stars
}

\author{
Ji Yeon Seok ${ }^{1,2}$ and Aigen $\operatorname{Li}^{1}$
}

\begin{abstract}
A distinct set of broad emission features at 3.3, 6.2, 7.7, 8.6, 11.3, and $12.7 \mu \mathrm{m}$, is often detected in protoplanetary disks (PPDs). These features are commonly attributed to polycyclic aromatic hydrocarbons (PAHs). We model these emission features in the infrared spectra of 69 PPDs around 14 T Tauri and 55 Herbig $\mathrm{Ae} / \mathrm{Be}$ stars in terms of astronomical-PAHs. For each PPD, we derive the size distribution and the charge state of PAHs. We then examine the correlations of the PAH properties (i.e., sizes and ionization fractions) with the stellar properties (e.g., stellar effective temperature, luminosity, and mass). We find that the characteristic size of PAHs shows a tendency of correlating with the stellar effective temperature $\left(T_{\text {eff }}\right)$ and interpret this as the preferential photodissociation of small PAHs in systems with higher $T_{\text {eff }}$ of which the stellar photons are more energetic. In addition, the PAH size shows a moderate correlation with the red-ward wavelength-shift of the $7.7 \mu \mathrm{m}$ PAH feature that is commonly observed in disks around cool stars. The ionization fraction of PAHs does not seem to correlate with any stellar parameters. This is because the charging of PAHs depends on not only the stellar properties (e.g., $T_{\text {eff }}$, luminosity) but also the spatial distribution of PAHs in the disks. The mere negative correlation between the PAH size and the stellar age suggests that continuous replenishment of PAHs via the outgassing of cometary bodies and/or the collisional grinding of planetesimals and asteroids is required to maintain the abundance of small PAHs against complete destruction by photodissociation.
\end{abstract}

Subject headings: circumstellar matter — infrared: stars — planetary systems: protoplanetary disks

\footnotetext{
${ }^{1}$ Department of Physics and Astronomy, University of Missouri, Columbia, MO 65211, USA, seokji@missouri.edu, lia@missouri.edu

${ }^{2}$ Key Laboratory of Optical Astronomy, National Astronomical Observatories, Chinese Academy of Sciences, Beijing 100012, China, jiseok@bao.ac.cn
} 


\section{Introduction}

A number of infrared (IR) observations of disks around low- and intermediate-mass premain-sequence stars clearly evince a distinct set of broad emission features at 3.3, 6.2, 7.7, 8.6, 11.2, and $12.7 \mu \mathrm{m}$ (e.g., Brooke et al. 1993; Meeus et al. 2001; Acke \& van den Ancker 2004; Habart et al. 2004a; Sloan et al. 2005; Geers et al. 2006, 2007a, 2007b; Keller et al. 2008; Acke et al. 2010; Maaskant et al. 2014). These IR spectral features are commonly attributed to polycyclic aromatic hydrocarbons (PAHs; Léger \& Puget 1984, Allamandola et al. 1985). While $\lesssim 10 \%$ of the disks around T Tauri stars (TTSs; $M_{\star} \lesssim 2 M_{\odot}$ ) show PAH features in their IR spectra (e.g., Furlan et al. 2006; Geers et al. 2006), for Herbig $\mathrm{Ae} / \mathrm{Be}(\mathrm{HAeBe})$ stars, the higher-mass counterparts of TTSs $\left(M_{\star} \sim 2-8 M_{\odot}\right)$, PAH features are more commonly detected from their disks (e.g., detection rate of $\sim 70 \%$, Acke et al. 2010). On the other hand, more evolved disks around main-sequence stars, so-called debris disks around Vega-type stars, hardly show PAH features. For instance, Chen et al. (2006) reported no detection of PAH emission in the IR spectra of 59 debris disks obtained with the Infrared Spectrograph (IRS) on board the Spitzer Space Telescope. This suggests that the characteristics of PAH emission of these protoplanetary disks (PPDs) are contingent on the stellar properties and evolutionary phases of the disks and the PAH features can be used to probe the physical and chemical properties of PPDs.

The PAH emission features are often used as a diagnostic tool of the physical conditions of PPDs and a tracer of the structure of PPDs. Their band ratios are sensitive to the charge state of PAHs, which is controlled by electron density, gas temperature, and starlight intensity (e.g., Bakes \& Tielens 1994; Weingartner \& Draine 2001). Due to their stochastic heating nature (Draine \& Li 2001), PAH emission features can be detected far away from the central star (e.g., the PAH features in the disk around WL 16, a HAeBe star, are spatially extended up to 440 AU from the central star, Ressler \& Barsony 2003) and can be spatially

more extended than the emission of large grains in thermal equilibrium with the stellar radiation. In addition, ionized PAHs could trace protoplanetary gaps (Maaskant et al. 2014).

PAHs profoundly influence the thermal budget and chemistry of PPDs, where planet(s) would newly form. PAH molecules in the surface layer of the disk, where PAHs are directly exposed to the stellar radiation, efficiently get photoionized and provide photoelectrons for heating the ambient gas (e.g., Kamp \& Dullemond 2004). PAHs have large surface areas for chemical reactions (e.g., Jonkheid et al. 2004; Habart et al. 2004b). In particular, PAHs can

alter carbon chemistry of the disk through transferring electrons from neutral and negatively charged PAHs to ionized carbon atoms. Also, PAHs have large far-ultraviolet (UV) opacity so that the inner parts of the disk can be shielded from the stellar UV radiation. In this context, examining the physical properties of PAHs in PPDs and their correlations with individual 
stellar properties of the PPDs would be essential to understand the diverse characteristics of PPDs.

Profuse spectroscopic observations obtained with IR space telescopes such as the Infrared Space Observatory (ISO) and/or Spitzer as well as ground-based telescopes such as the Very Large Telescope (VLT) or the NASA Infrared Telescope Facility (IRTF) have been utilized to conduct various studies of PAH features in PPDs, in particular using a large number of sample. Acke \& van den Ancker (2004) presented ISO spectra of a sample of 46 HAeBe stars and detect PAH features in 27 sources of their sample $(\sim 57 \%)$. Later, using Spitzer/IRS data, Geers et al. (2006) analyzed the PAH features of 38 TTSs in comparison with a sample of HAeBe stars and found that the 6.2 and $11.3 \mu \mathrm{m}$ PAH features are commonly detected while those at 7.7 and $8.6 \mu \mathrm{m}$ are relatively rare due to the strong $9.7 \mu \mathrm{m}$ silicate emission. As PAH features are often strong in HAeBe stars, a number of previous studies focus on the PAH features of HAeBe stars; Sloan et al. (2005) found that the ionization fraction of PAHs is higher for PPDs with hotter and brighter central stars. Keller et al. (2008) used Spitzer/IRS spectra of 22 targets including 18 HAeBe stars and reported spectral variations among these stars, which is associated with the effective temperature of the host stars. With a larger sample of HAeBe stars (i.e., 53 Herbig Ae stars), Acke et al. (2010) pointed out that the diversity of PAH emission features shown in the Herbig Ae stars is mainly attributed to chemical processing induced by the stellar UV radiation field. In addition, spatially resolved spectroscopy using ground-based telescopes has revealed that the PAH features in some PPDs around TTSs and HAeBe stars are spatially extended over a few tens to hundred AU (e.g., van Boekel et al. 2004; Habart et al. 2006; Geers et al. 2007b; Maaskant et al. 2014), and the observed PAH extent does not always coincide with that of the dust continuum (e.g., Geers et al. 2007b; Maaskant et al. 2014).

While the characteristics of PAH emission features and their band ratios have been extensively studied for a number of PPDs in the literature, the physical properties of PAH molecules in the PPDs are still mostly unknown, which requires detailed modeling of PAH spectra. Habart et al. (2004a) performed a disk modeling using a radiation transfer code assuming either fully ionized or neutral PAHs with a single PAH size (i.e., $N_{\mathrm{C}}=40$ or 100). They compared the model results with observations of HAeBe stars from $I S O$ and ground-based telescopes but limited it to the band strengths (i.e., not aim to reproduce the PAH features of individual sources). Later, Maaskant et al. (2014) implemented a model to calculate the charge state of PAHs in a radiative transfer code and applied it to the PAH spectra of four PPDs. Since they intended to verify the effect of ionization, a single PAH size is adopted. However, the typical size of PAHs in the disk might vary substantially from one object to another and could affect the characteristics of PAH spectral features. 
In previous papers, we performed comprehensive modelings of the PAH features for three specific sources (HD141569A: Li \& Lunine 2003b; HD 34700: Seok \& Li 2015; HD 169142: Seok \& Li 2016) adopting the astro-PAH model of Li \& Draine (2001b) and Draine \& Li (2007) and derived the properties of PAHs in the disks. Since, only for limited sources, their PAH properties have been well-constrained so far, an analysis with a statistically relevant sample is further required to probe the possible physical association between PAHs in PPDs and their ambient conditions. In this paper, we present the PAH spectra of 69 PPDs collated from the literature and compile the fundamental stellar properties such as the effective temperature, stellar mass, and age to interpret the PAH properties including the size and charge state of PAHs. By coherently modeling the PAH spectra of a large number of sources including both TTSs and HAeBe stars and statistically examining their PAH properties, we are able to characterize the physical and chemical properties of PAHs in the PPDs and reveal their relations with the stellar properties and disk environments.

The paper is organized as follows. The sample selection and observational data are summarized in Section 2, and the astro-PAH model adopted here and the model-fitting procedure are described in Section 3. We present the model results and statistics in Section 4 . In Section 5, we discuss the correlations between the PAH properties and stellar parameters, the destruction of PAHs in PPDs, the aliphatic fraction of PAH molecules, and the effects of different methods of extracting the PAH spectra. Finally, we summarize the main results in Section 6.

\section{Data}

\subsection{Sample Selection}

We search for PPDs reported to show PAH emission in the literature, and 69 disks are found, consisting of 14 TTSs (Furlan et al. 2006; Geers et al. 2006, 2007b; Keller et al. 2008; Schütz et al. 2009; Merín et al. 2010) and 55 HAeBe stars (Acke \& van den Ancker 2004; Sloan et al. 2005, 2007; Keller et al. 2008; Boersma et al. 2009; Schütz et al. 2009; Acke et al. 2010; Kóspál et al. 2012; Maaskant et al. 2013), which allow us to cover a wide range of stellar parameters. To the best of our knowledge, this is the largest sample of PPDs showing

PAH features in their IR spectra, which are to be modeled taking precise PAH chemistry into account. The final sample with their stellar parameters is listed in Table 1 . 


\subsection{Data Set}

For the 69 sources, we collate both near-IR (NIR; $\lesssim 4 \mu \mathrm{m})$ and mid-IR (MIR; $\gtrsim 5 \mu \mathrm{m})$ spectra available in the literature to analyze the major PAH features at $3.3-11.3 \mu \mathrm{m}$. This is important because a simultaneous modeling of as many PAH bands as possible can more accurately constrain the properties of PAHs in a disk although it is the $6.2,7.7$, and $8.6 \mu \mathrm{m}$ features that mainly control the best-fit model parameters. For the MIR data, the Spitzer/IRS spectra (SL module: $R \approx 60-127$ at $5.2-14.5 \mu \mathrm{m}$ ) of all except 11 sources are available in the literature (e.g., Furlan et al. 2006; Geers et al. 2006; Kessler-Silacci et al. 2006; Keller et al. 2008; Acke et al. 2010; Merín et al. 2010; Kóspál et al. 2012; Maaskant et al. 2013). Among those with Spitzer/IRS data, the IRS spectra of 8 sources do not sufficiently cover the wavelength range of the SL module for viable model calculation. For four of them (HD 100546, HD 163296, HD 179218, and Wray 15-1484), we take fully processed ISO/SWS spectra (2.38-45.2 $\mu \mathrm{m}, R \sim 1500-2000)$ from the $I S O /$ SWS atlas (Sloan et al. 2003) 1 For SR $21 \mathrm{~N}$, we adopt the $N$-band spectrum $(7.7-12.5 \mu \mathrm{m}, R \sim 350)$ obtained with the VLT Imager and Spectrometer for the mid-IR (VISIR) installed at the VLT from Geers et al. (2007b). For the remaining three sources (HD 98922, HD 259431, and T Cha), no other supplementary data are available in the literature. While more than two PAH bands are present in the IRS spectrum of HD 98922, only the $11.3 \mu \mathrm{m}$ feature is covered by the spectra of HD 259431 and T Cha. We exclude the latter two sources for model calculations. For those without Spitzer/IRS data, we take the ISO/ISOPHOT-SL spectra $(5.8-11.6 \mu \mathrm{m}$, $R \approx 100$ ) of Kóspál et al. (2012)2 for three sources (IRAS 03260+3111, VX Cas, and WL $16)$ the $N$-band spectrum $(8-13 \mu \mathrm{m})$ obtained with the ESO TIMMI2 camera at La Silla Observatory for PDS 144N (Schütz et al. 2009), and the ISO/SWS spectra of eight sources (BD+404124, HD 200775, IRAS 06084-0611, LkH $\alpha$ 224, MWC 297, MWC 865, MWC 1080, and TY CrA) from the ISO/SWS atlas (Sloan et al. 2003).

Unlike the MIR spectra, the NIR spectra are not available for all sources. For the 27 sources observed by ISO/SWS, we adopt the SWS spectra from the ISO/SWS atlas. For IRAS 03260+3111, VX Cas, WL 16, HD 97300, RR Tau, and WW Vul, we take the ISOPHOT-SS $(2.5-4.9 \mu \mathrm{m}, R \approx 100)$ data from Kóspál et al. (2012). For AK Sco and BF Ori (Acke \& van den Ancker 2006), Oph IRS48 (Geers et al. 2007a), HD 98922, HD 101412, HD 141569, SR21N, T Cha, and VV Ser (Geers et al. 2007b), we collect the $L$-band (2.8-4.2 $\mu \mathrm{m})$ spectra obtained with ISAAC, the Infrared Spectrometer And Array Camera, installed at the VLT. For HD 34700, we use the $L$-band $(\sim 1.9-4.2 \mu \mathrm{m})$ spectrum obtained with the

${ }^{1}$ http://isc.astro.cornell.edu/ sloan/library/swsatlas/atlas.html

${ }^{2}$ http://vizier.cfa.harvard.edu/viz-bin/VizieR?-source=J/ApJS/201/11 
medium-resolution spectrograph SpeX at the IRTF (Smith et al. 2004).

When we use two different observational sets for the NIR and MIR spectra of one object, we need to match their flux levels. If there is an overlapping wavelength range of the two spectra (i.e., ISO/SWS or ISOPHOT and Spitzer/IRS data for the NIR and MIR spectra, respectively), we derive a scaling factor using the overlapping part and scale the NIR spectrum with respect to the MIR spectrum 3 If no overlapping part is available, for those with an NIR spectrum from a ground-based telescope and an MIR spectrum from Spitzer, we scale both spectra with respect to the photometric measurements taken from VizieR 4

In summary, we accumulate the IR spectroscopic data of 69 disks from the literature and data archives, composed of 43 NIR and 69 MIR spectra. Table 1 presents a summary of the data set compiled in this work.

\footnotetext{
${ }^{3}$ We scaled the NIR spectra (i.e., ISO data) with respect to the MIR spectra (i.e., Spitzer data) because the Spitzer spectra are mostly consistent with the IR photometry whereas the ISO spectra of some objects are rather noisy and/or have large uncertainties in their absolute flux calibration.

${ }^{4}$ http://vizier.u-strasbg.fr/vizier
} 
Table 1. Sample of Protoplanetary Disks with PAH Emission

\begin{tabular}{|c|c|c|c|c|c|c|c|c|c|}
\hline \multirow[t]{2}{*}{ Object } & \multirow{2}{*}{$\begin{array}{l}T_{\text {eff }} \\
(\mathrm{K})\end{array}$} & \multirow{2}{*}{$\begin{array}{c}L_{\star} \\
\left(L_{\odot}\right)\end{array}$} & \multirow{2}{*}{$\begin{array}{c}M_{\star} \\
\left(M_{\odot}\right)\end{array}$} & \multirow{2}{*}{$\begin{array}{c}\text { Age } \\
(\mathrm{Myr})\end{array}$} & \multirow{2}{*}{$\begin{array}{c}d \\
(\mathrm{pc})\end{array}$} & \multirow[t]{2}{*}{ Ref. } & \multicolumn{2}{|c|}{ Data $^{a}$} & \multirow[t]{2}{*}{$9.7 \mu \mathrm{m}^{\mathrm{b}}$} \\
\hline & & & & & & & NIR & MIR & \\
\hline AB Aur & 9800 & 57.5 & 2.5 & 3.7 & 139 & (1) & SWS & A10 & em \\
\hline AK Sco & 6500 & 8.9 & 1.66 & 9.3 & 103 & (1) & A06 & A10 & $\mathrm{em}$ \\
\hline $\mathrm{BD}+40^{\circ} 4124$ & 22000 & 5900 & $\gtrsim 5.99$ & $\lesssim 0.01$ & 980 & $(2),(3),(4)$ & SWS & SWS & non \\
\hline BF Ori & 8750 & 56 & 2.58 & 3.15 & 375 & (1) & A06 & K12 & $\mathrm{em}$ \\
\hline DoAr 21 & 5080 & 7.5 & 2.40 & 0.4 & 120 & $(5),(6),(7)$ & SWS & M10 & abs \\
\hline EC 82 & 4060 & 3.21 & 0.75 & 1.5 & 415 & $(8),(9)$ & $\ldots$ & K06 & em \\
\hline HD 31648 & 8200 & 15.1 & 1.93 & 7.8 & 137 & (1) & SWS & A10 & em \\
\hline HD 34282 & 8625 & 13.5 & 1.59 & 6.4 & 191 & (1) & SWS & A10 & non \\
\hline HD 34700 & 6000 & 20.4 & 1.20 & 10 & 260 & (10) & SpX & K12 & non \\
\hline HD 35187 & 8900 & 14.1 & 1.93 & 10.7 & 114 & (1) & SWS & A10 & $\mathrm{em}$ \\
\hline HD 36112 & 7800 & 66 & 2.90 & 2.1 & 279 & (1) & $\ldots$ & A10 & em \\
\hline HD 36917 & 10000 & 245.5 & 3.98 & 0.72 & 375 & (1) & $\ldots$ & $\mathrm{A} 10$ & em \\
\hline HD 37357 & 9250 & 52.5 & 2.48 & 3.7 & 375 & $(1)$ & & A10 & em \\
\hline HD 37411 & 9100 & 34.4 & 1.90 & 9 & 510 & $(11),(12),(13)$ & & A10 & non \\
\hline HD 37806 & 11000 & 282 & 3.94 & 0.88 & 375 & (1) & $\ldots$ & A10 & em \\
\hline HD 38120 & 11000 & 41.7 & 2.49 & 5.1 & 375 & (1) & $\ldots$ & A10 & em \\
\hline HD 58647 & 10500 & 912 & 6.0 & 1 & 543 & (14) & $\ldots$ & $\mathrm{A} 10$ & em \\
\hline HD 72106 & 11000 & 21.9 & 2.40 & 9 & 289 & (1) & & $\mathrm{A} 10$ & $\mathrm{em}$ \\
\hline HD 85567 & 20900 & 14791 & 12.0 & $\ldots$ & 1500 & (15) & $\ldots$ & A10 & em \\
\hline HD 95881 & 9000 & 7.6 & 1.7 & $\gtrsim 3.16$ & 118 & (16) & SWS & $\mathrm{A} 10$ & $\mathrm{em}$ \\
\hline HD 97048 & 10000 & 44 & 2.53 & 4.61 & 150 & (3) & SWS & A10 & non \\
\hline HD 97300 & 10700 & 37 & 2.50 & $\gtrsim 3$ & 188 & $(17),(18)$ & PHT & K12 & non \\
\hline HD 98922 & 10500 & 5888 & $\gtrsim 4.95$ & $\lesssim 0.01$ & 1150 & $(1),(4)$ & G07 & A10 & $\mathrm{em}$ \\
\hline HD 100453 & 7600 & 10 & 1.8 & 15 & 114 & $(14)$ & SWS & A10 & non \\
\hline HD 100546 & 10500 & 32 & 2.4 & $\gtrsim 10$ & 103 & (16) & SWS & SWS & $\mathrm{em}$ \\
\hline HD 101412 & 8600 & 83 & 3.0 & 1.2 & 600 & (19) & G07 & $\mathrm{A} 10$ & $\mathrm{em}$ \\
\hline HD 135344B & 6750 & 14.5 & 1.9 & 6.6 & 142 & (1) & SWS & $\mathrm{A} 10$ & non \\
\hline HD 139614 & 7600 & 12.6 & 1.76 & 8.8 & 142 & (1) & SWS & A10 & $\mathrm{em}$ \\
\hline HD 141569 & 9800 & 30.9 & 2.33 & 5.7 & 116 & (1) & G07 & K12 & non \\
\hline HD 142527 & 6360 & 23.58 & 2.3 & 2 & 140 & $(6),(20)$ & SWS & $\mathrm{A} 10$ & $\mathrm{em}$ \\
\hline HD 142666 & 7900 & 27.5 & 2.15 & 5 & 145 & (1) & SWS & A10 & em \\
\hline HD 144432 & 7500 & 19.1 & 1.95 & 6.4 & 145 & (1) & SWS & K12 & $\mathrm{em}$ \\
\hline HD 145718 & 8100 & 19.5 & 1.93 & 7.4 & 145 & (1) & $\ldots$ & K08 & em \\
\hline HD 163296 & 9200 & 33 & 2.23 & 5.1 & 119 & (1) & SWS & SWS & em \\
\hline HD 169142 & 8250 & 8.55 & 1.69 & 6 & 145 & (21) & SWS & A10 & non \\
\hline HD 179218 & 9640 & 182 & 3.66 & 1.08 & 254 & (1) & SWS & SWS & $\mathrm{em}$ \\
\hline HD 200775 & 18600 & 8912.5 & 10.7 & 0.016 & 429 & (1) & SWS & SWS & em \\
\hline
\end{tabular}


Table 1 - Continued

\begin{tabular}{|c|c|c|c|c|c|c|c|c|c|}
\hline \multirow[t]{2}{*}{ Object } & \multirow{2}{*}{$\begin{array}{l}T_{\text {eff }} \\
(\mathrm{K})\end{array}$} & \multirow{2}{*}{$\begin{array}{c}L_{\star} \\
\left(L_{\odot}\right)\end{array}$} & \multirow{2}{*}{$\begin{array}{c}M_{\star} \\
\left(M_{\odot}\right)\end{array}$} & \multirow{2}{*}{$\begin{array}{l}\text { Age } \\
(\mathrm{Myr})\end{array}$} & \multirow{2}{*}{$\begin{array}{c}d \\
(\mathrm{pc})\end{array}$} & \multirow[t]{2}{*}{ Ref. } & \multicolumn{2}{|c|}{ Data $^{a}$} & \multirow[t]{2}{*}{$9.7 \mu \mathrm{m}^{\mathrm{b}}$} \\
\hline & & & & & & & NIR & MIR & \\
\hline HD 244604 & 8200 & 55 & 2.66 & 2.79 & 375 & (1) & & A10 & $\mathrm{em}$ \\
\hline HD 250550 & 11000 & 138 & 3.1 & 1.42 & 700 & $(15),(13)$ & $\ldots$ & A10 & $\mathrm{em}$ \\
\hline HD 259431 & 14000 & 2239 & 7.1 & 0.059 & 660 & (1) & & $\mathrm{A} 10$ & non \\
\hline HD 281789 & 9520 & $10^{\mathrm{c}}$ & $\ldots$ & $\ldots$ & 350 & $(22)$ & & K08 & $\mathrm{em}$ \\
\hline IC348 LRL110 & 3778 & 0.22 & 0.78 & $\ldots$ & 250 & (23) & & M10 & em \\
\hline IC348 LRL190 & 3306 & 0.09 & 0.35 & $\ldots$ & 250 & $(23)$ & & M10 & $\mathrm{em}$ \\
\hline IRAS $03260+3111$ & 13000 & $\sim 180^{\mathrm{d}}$ & & $\ldots$ & 290 & $(24),(25)$ & PHT & PHT & non \\
\hline IRAS 06084-0611 & $18600^{\mathrm{e}}$ & $10^{\mathrm{c}}$ & $\ldots$ & $\ldots$ & 1050 & $(26)$ & SWS & SWS & non \\
\hline J032903.9+305630 & 5630 & 0.05 & $\ldots$ & $\ldots$ & 250 & (23) & $\ldots$ & M10 & abs \\
\hline J182858.1+001724 & 5830 & 4.76 & 1.40 & $\ldots$ & 260 & (23) & & M10 & non \\
\hline J182907.0+003838 & 4060 & 0.6 & 1.04 & $\ldots$ & 260 & $(23)$ & & M10 & em \\
\hline $\mathrm{LkH} \alpha 224$ & 7850 & 115 & 6.05 & 0.312 & 980 & $(2),(3)$ & SWS & SWS & non \\
\hline $\mathrm{LkH} \alpha 330$ & 5800 & 11 & 2.50 & 3 & 250 & $(5),(6),(27)$ & 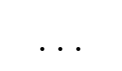 & G06 & $\mathrm{em}$ \\
\hline MWC 297 & 24000 & 10230 & 9.0 & 1 & 250 & $(2),(3)$ & SWS & SWS & abs \\
\hline MWC 865 & 14000 & 8710 & 9.0 & 1 & 400 & $(28)$ & SWS & SWS & non \\
\hline MWC 1080 & 30000 & 180000 & 10.0 & 1 & 2200 & $(2),(3)$ & SWS & SWS & non \\
\hline Oph IRS48 & 10000 & 14.3 & 2.25 & 15 & 120 & $(11),(9)$ & G07 & M13 & non \\
\hline PDS 144N & 8750 & $10^{\mathrm{c}}$ & $\ldots$ & $\ldots$ & 1000 & (29) & $\cdots$ & TMM & non \\
\hline RR Tau & 8460 & 2 & 3.57 & 1.34 & 160 & $(2),(3)$ & PHT & A10 & non \\
\hline RXJ1615.3-3255 & 4590 & 0.85 & 1.28 & $\ldots$ & 120 & (23) & & M10 & $\mathrm{em}$ \\
\hline SR $21 \mathrm{~N}$ & 5830 & 6.5 & 1.70 & 1 & 125 & $(6),(30)$ & G07 & VSR & non \\
\hline SU Aur & 5860 & 11 & 1.88 & 6.3 & 146 & $(5),(31)$ & $\ldots$ & K08 & $\mathrm{em}$ \\
\hline T Cha & 5250 & 1.34 & 1.10 & 7 & 108 & $(6),(32)$ & G07 & G06 & non \\
\hline TY CrA & 12000 & 98 & 3.16 & 3 & 140 & (33) & SWS & SWS & non \\
\hline UX Tau & 5520 & 3.5 & 1.50 & 3.0 & 140 & $(34),(35)$ & & F06 & em \\
\hline V590 Mon & 13000 & 295 & 4.71 & 2.8 & 800 & $(36)$ & & A10 & $\mathrm{em}$ \\
\hline V892 Tau & 8000 & 21 & 2.06 & 6.11 & 140 & (3) & SWS & K08 & $\mathrm{em}$ \\
\hline VV Ser & 14000 & 324 & 4.0 & 0.64 & 260 & (1) & G07 & $\mathrm{A} 10$ & em \\
\hline VX Cas & 9500 & 60 & 2.55 & 3.4 & 620 & (1) & PHT & PHT & em \\
\hline WL 16 & 9000 & 250 & 4.0 & 1 & 125 & (37) & PHT & PHT & non \\
\hline Wray 15-1484 & 30000 & 977 & $\ldots$ & $\ldots$ & 750 & $(2)$ & SWS & SWS & non \\
\hline WW Vul & 9000 & 170 & 3.70 & 0.9 & 700 & (1) & PHT & K12 & em \\
\hline
\end{tabular}

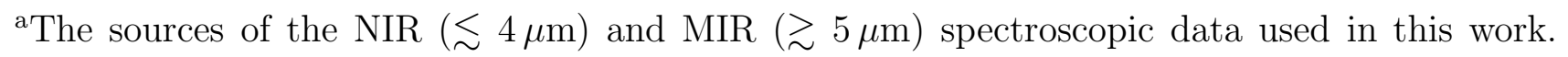
A06: VLT/ISAAC data from Acke \& van den Ancker (2006), A10: Spitzer/IRS data from Acke 
et al. (2010), F06: Spitzer/IRS data from Furlan et al. (2006), G06: Spitzer/IRS data from Geers et al. (2006), G07: VLT/ISAAC data from Geers et al. (2007a, 2007b), K06: Spitzer/IRS data from Kessler-Silacci et al. (2006), K08: Spitzer/IRS data from Keller et al. (2008), K12: Spitzer/IRS data from Kóspál et al. (2012), M10: Spitzer/IRS data from Merín et al. (2010), M13: Spitzer/IRS data from Maaskant et al. (2013), PHT: ISO/ISOPHOT-S data from Kóspál et al. (2012), SpX: IRTF/SpeX data from Smith et al. (2004), SWS: Fully processed ISO/SWS atlas (http://isc.astro.cornell.edu/ sloan/library/swsatlas/atlas.html, Sloan et al. 2003), TMM: ESO/TIMMI2 data from Schütz et al. (2009), and VSR: VLT/VISIR data from Geers et al. (2007b).

${ }^{\mathrm{b}}$ Note for the presence or absence of the $9.7 \mu \mathrm{m}$ silicate spectral feature in the disk. If present, we label it "em" if it is detected in emission or "abs" if in absorption. The symbol "non" indicates the disk does not exhibit the $9.7 \mu \mathrm{m}$ silicate feature either in emission or in absorption.

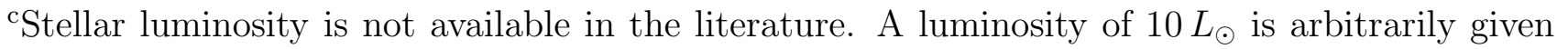
for modeling, which does not affect the model results.

${ }^{\mathrm{d}} L_{\star}$ of IRAS $03260+3111$ is roughly estimated from $L_{\mathrm{tot}}-L_{\mathrm{bol}}$, where $L_{\text {tot }}=318 L_{\odot}$ and $L_{\mathrm{bol}}=138 L_{\odot}$ are taken from Harvey et al. (1984) and Connelley et al. (2008), respectively.

${ }^{\mathrm{e}} T_{\text {eff }}$ of IRAS 06084-0611 is not well constrained in the literature. We adopt the typical $T_{\text {eff }}$ (18,600 K) for its spectral type (B3 for IRAS 06084 VLA4; Boersma et al. 2009). Unless the spectral type significantly differs, the model results would not be altered much.

Note. - "..." indicates that the stellar parameters or the NIR data are not available in the literature.

References. - (1) Alecian et al. (2013), (2) Acke \& van den Ancker (2004), (3) Alonso-Albi et al. (2009), (4) Manoj et al. (2006), (5) Menu et al. (2015), (6) van der Marel et al. (2016), (7) Jensen et al. (2009), (8) Rigliaco et al. (2015), (9) Sturm et al. (2013), (10) Seok \& Li (2015), (11) Maaskant et al. (2014), (12) Juhász et al. (2010), (13) Fairlamb et al. (2015), (14) Mariñas et al. (2011), (15) Verhoeff et al. (2012), (16) van Boekel et al. (2005), (17) Siebenmorgen et al. (2000), (18) Hubrig et al. (2009), (19) Folsom et al. (2012), (20) Fukagawa et al. (2010), (21) Seok \& Li (2016), (22) Keller et al. (2008), (23) Merín et al. (2010), (24) Harvey et al. (1984), (25) Kóspál et al.(2012), (26) Boersma et al. (2009), (27) Brown et al. (2008), (28) Borges Fernandes et al. (2007), (29) Schütz et al. (2009), (30) Prato et al. (2003), (31) Jeffers et al. (2013), (32) Huélamo et al. (2011), (33) Casey et al. (1998), (34) Pinilla et al. (2014), (35) Magazzu et al. (1991), (36) Liu et al. (2011), (37) Ressler \& Barsony (2003) 


\subsection{Extraction of the PAH Emission Spectrum}

Since we aim to mainly model the PAH emission features, for each disk, we need to extract the PAH features from the original data by subtracting the underlying continuum. The data from Acke et al. (2010) are already continuum-subtracted, with the dust thermal continuum emission fitted with a spline function.Similarly, Keller et al. (2008) extracted the PAH features by fitting a spline with two sets of anchor points for the spectra with or without silicate emission. Following these two previous studies, we take a similar approach to the rest of the data that we have collected to obtain continuum-subtracted spectra (hereafter residual spectra).

Acke et al. (2010) split the IRS spectrum into two wavelength ranges (5-7 and 7-14 $\mu \mathrm{m})$ and treated them differently. The former range, containing the $6.2 \mu \mathrm{m}$ PAH feature, has a relatively smooth continuum underneath the feature, so it is easy to reproduce the continuum with a spline through a few anchor points. We use a cubic spline through the anchor points at 5.35, 5.45, 5.58, 6.66, 7.06, 7.40, 7.55, and $7.70 \mu \mathrm{m}$, adopted from Acke et al. (2010) for the 5-7 $\mu \mathrm{m}$ interval, with a slight shift to avoid peculiar spectral features appearing in individual spectra. The latter range, however, is not as simple as the former, especially for the sources whose spectra are dominated by the strong $9.7 \mu \mathrm{m}$ silicate emission (or absorption) feature. Following Keller et al. (2008), we use two sets of anchor points for the spectra dominated by PAH bands (PAH-dominated sources) and those dominated by the $9.7 \mu \mathrm{m}$ silicate emission or absorption band. For PAH-dominated sources, we adopt anchor points at 5.55, 5.80, 6.7, $7.0,9.15,9.45,9.7,10.2,10.7,11.8,12.2,13.05,13.25,13.8,14.0$, and $14.8 \mu \mathrm{m}$. Like the 5-7 $\mu \mathrm{m}$ interval, we slightly adjust the anchor points, but for most cases, the variations are marginal. For the silicate emission (or absorption) sources, we use anchor points at 7.35, 8.95, and $9.35 \mu \mathrm{m}$ instead of those at 9.15 and $9.45 \mu \mathrm{m}$ while keeping other points the same as those for PAH-dominated sources. As pointed out by Acke et al. (2010), spline fits result in considerable extra residuals (especially between 7-9 $\mu \mathrm{m}$ ) for those with strong silicate emission and weak PAH features. Also, some objects (e.g., BF Ori, EC 82, HD 144432, and WW Vul) show a crystalline silicate feature at $11.2-11.3 \mu \mathrm{m}$, which overlaps with the $11.2 \mu \mathrm{m}$ PAH feature. We subtract this feature by fitting the blended $11.3 \mu \mathrm{m}$ feature with two Drude profiles.

A continuum underlying the PAH emission feature at $3.3 \mu \mathrm{m}$ does not show a strong curvature for most cases, which is dominated by starlight with a possible contribution from a hot dust component. Since a minor feature at $3.43 \mu \mathrm{m}$, generally attributed to the $\mathrm{C}-\mathrm{H}$ vibrational modes in aliphatic hydrocarbons (e.g., Chiar et al. 2000; Pendleton \& Allamandola 2002), has a broad emission plateau blended with the $3.3 \mu \mathrm{m}$ PAH feature, we use the short- and long-wavelength sides of the 3.3 and $3.43 \mu \mathrm{m}$ features to fit the continuum using 
a linear function. We consider a wavelength range of 3.05-3.15 and 3.6-3.8 $\mu \mathrm{m}$, respectively, for the short- and long-wavelength sides. If HI recombination lines such as $\mathrm{Pf} \gamma$ at $3.741 \mu \mathrm{m}$ are strong, we exclude them from the fit. For a few sources (Oph IRS48, SR 21N, WL16, and V892 Tau), we use a second or fourth degree polynomial function as their NIR spectra seem to have a recognizable curvature.

There is one caveat for continuum-subtraction using a spline: it essentially forces the baselines between the PAH features in the residual spectrum to be zero, which might oversubtract the continuum. Since PAH emission features, which can be described by a combination of Drude profiles, have a considerably wide wing, even a spectrum consisting of PAH emission features only does not have a zero-level baseline. To investigate this effect of subtraction of a spline fit, we use the PAHFIT decomposition tool (Smith et al. 2007), which fits simultaneously the PAH features, dust and stellar continuum, and atomic and molecular emission lines, to fit the spectra of selected objects in our sample. Since PAHFIT uses blended Drude profiles to fit the PAH features, it results in non-zero baselines between the features. Although the residual spectra from the spline fit and PAHFIT result in some differences in the spectra, we find these differences do not alter our main result significantly (see Section 5.5). Thus, we keep the spline fit to extract the residual spectra for our analysis, and detailed comparisons will be discussed in Section 5.5 .

The residual spectra of the 69 sources are shown in Figures 119. The eight sources in Figure 9 are those excluded for further model calculations. The original spectra of these sources either have the limited wavelength coverage (e.g., HD 259431 and T Cha, see Section 2.2) or low signal-to-noise ratios $(\mathrm{S} / \mathrm{N})$, so their residual spectra are inadequate to perform model calculations.

\section{Model}

To derive the PAH properties from the residual spectra, we adopt the astro-PAH model of Li \& Draine (2001b) and Draine \& Li (2007). We assume a log-normal distribution function $\left(d n_{\mathrm{PAH}} / d a\right)$ for the $\mathrm{PAH}$ size distribution,

$d n_{\mathrm{PAH}} / d a=\frac{1}{\sqrt{\pi / 2} \sigma\left\{1-\operatorname{erf}\left[\ln \left(a_{\mathrm{min}}^{\mathrm{PAH}} / a_{0}\right) / \sqrt{2} \sigma\right]\right\}} \frac{1}{a} \exp \left\{-\frac{1}{2}\left[\frac{\ln \left(a / a_{0}\right)}{\sigma}\right]^{2}\right\}$, for $a>a_{\min }^{\mathrm{PAH}}$,

where $a_{0}$ and $\sigma$ are, respectively, the peak and the width of the log-normal distribution, and

$a_{\mathrm{min}}^{\mathrm{PAH}}$ is the lower cutoff of the PAH size. We set $a_{\mathrm{min}}^{\mathrm{PAH}} \equiv 3.5 \AA$, equivalent to a PAH molecule containing $\sim 20$ carbon atoms (i.e., $N_{\mathrm{C}} \approx 20$ ), which is the minimum size of PAHs required 


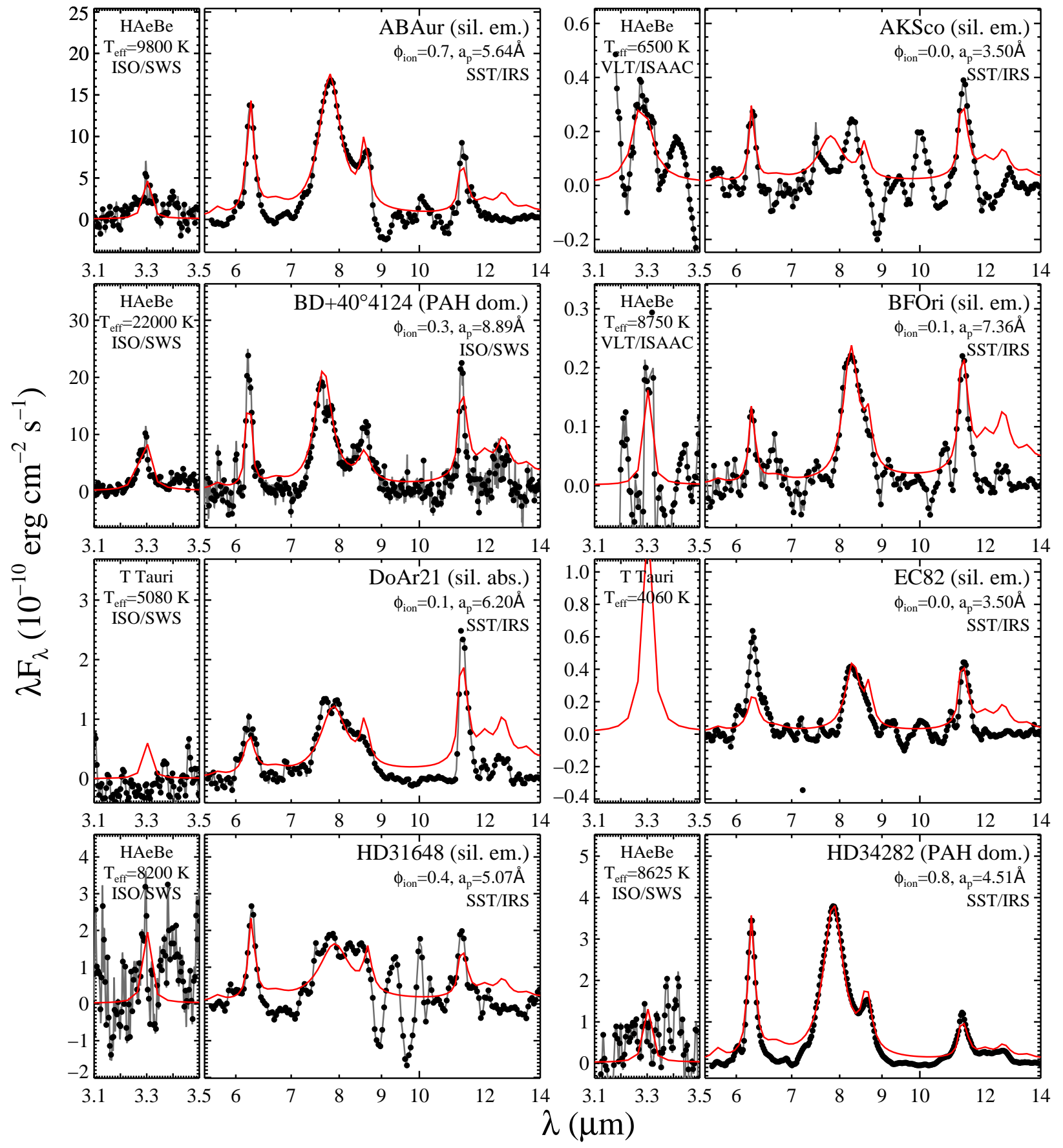

Fig. 1.- Comparison of the best-fit model spectra (red lines) with the residual spectra (i.e., continuumsubtracted spectra; grey lines) of AB Aur, AK Sco, BD $+40^{\circ} 4124$, BF Ori, DoAr 21, EC 82, HD 31648, and HD 34282. The interpolated data points actually used for model calculations are overlaid with black dots. The best-fit model parameters $\left(\phi_{\mathrm{ion}}\right.$ and $\left.a_{\mathrm{p}}\right)$ are given in the upper-right side of each panel. The label "PAH dom." specifies that the IR spectrum of the source is dominated by PAH features, while the label "sil. em." or "sil. abs." denotes that the source emits or absorbs strongly at the $9.7 \mu \mathrm{m}$ silicate feature, respectively. For each source, we also specify the nature of the central star (i.e., HAeBe or T Tauri), the stellar effective temperature $\left(T_{\text {eff }}\right)$, and the instruments used to obtain the observed spectrum (e.g., ISO/SWS, Spitzer/IRS [shortened as SST/IRS], VLT/ISAAC, etc.). 

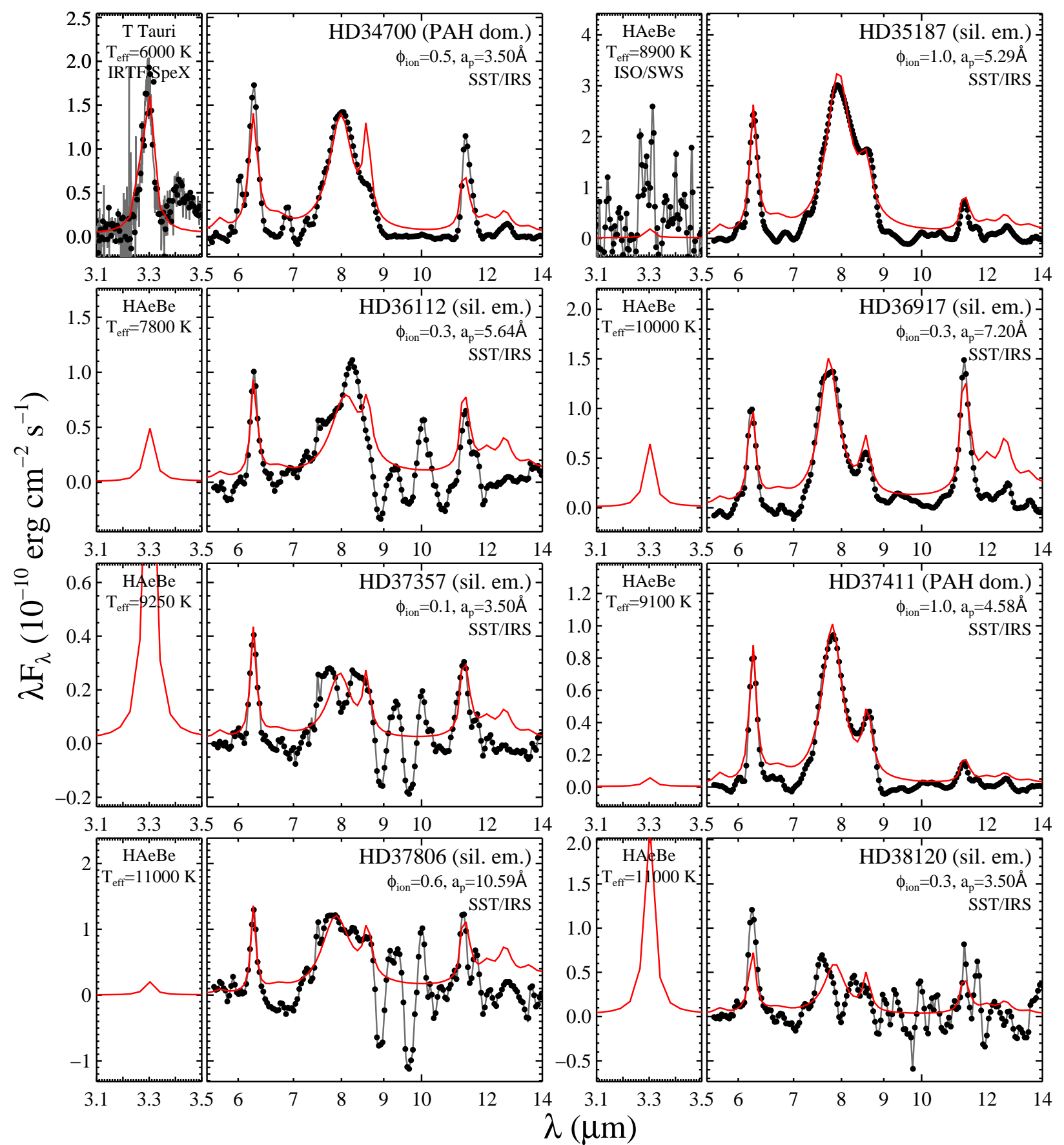

Fig. 2.- Same as Figure 1 but for HD 34700, HD 35187, HD 36112, HD 36917, HD 37357, HD 37411, HD 37806, and HD 38120. 

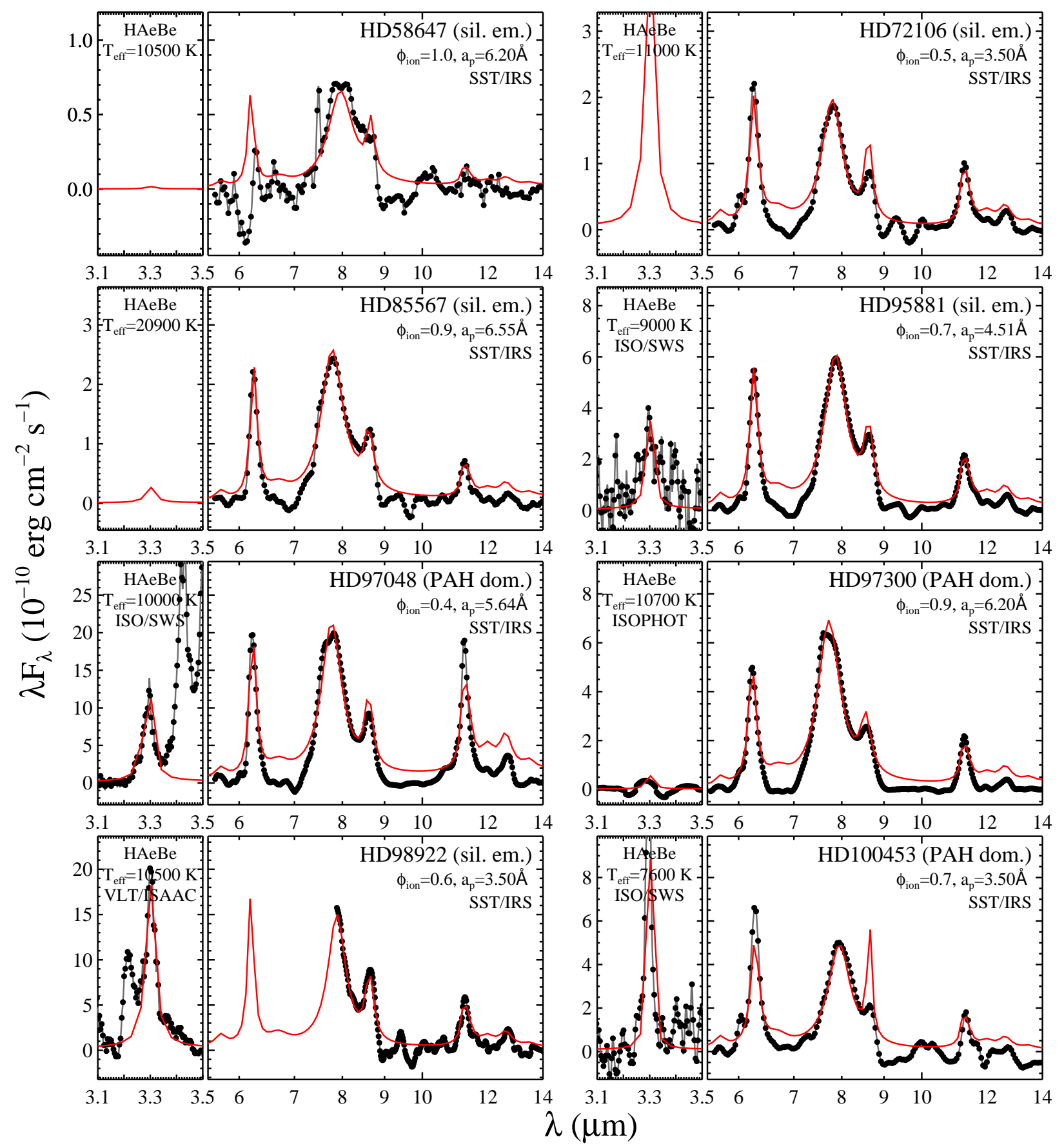

Fig. 3.- Same as Figure 1 but for HD 58647, HD 72106, HD 85567, HD 95881, HD 97048, HD 97300, HD 98922, and HD 100453. 


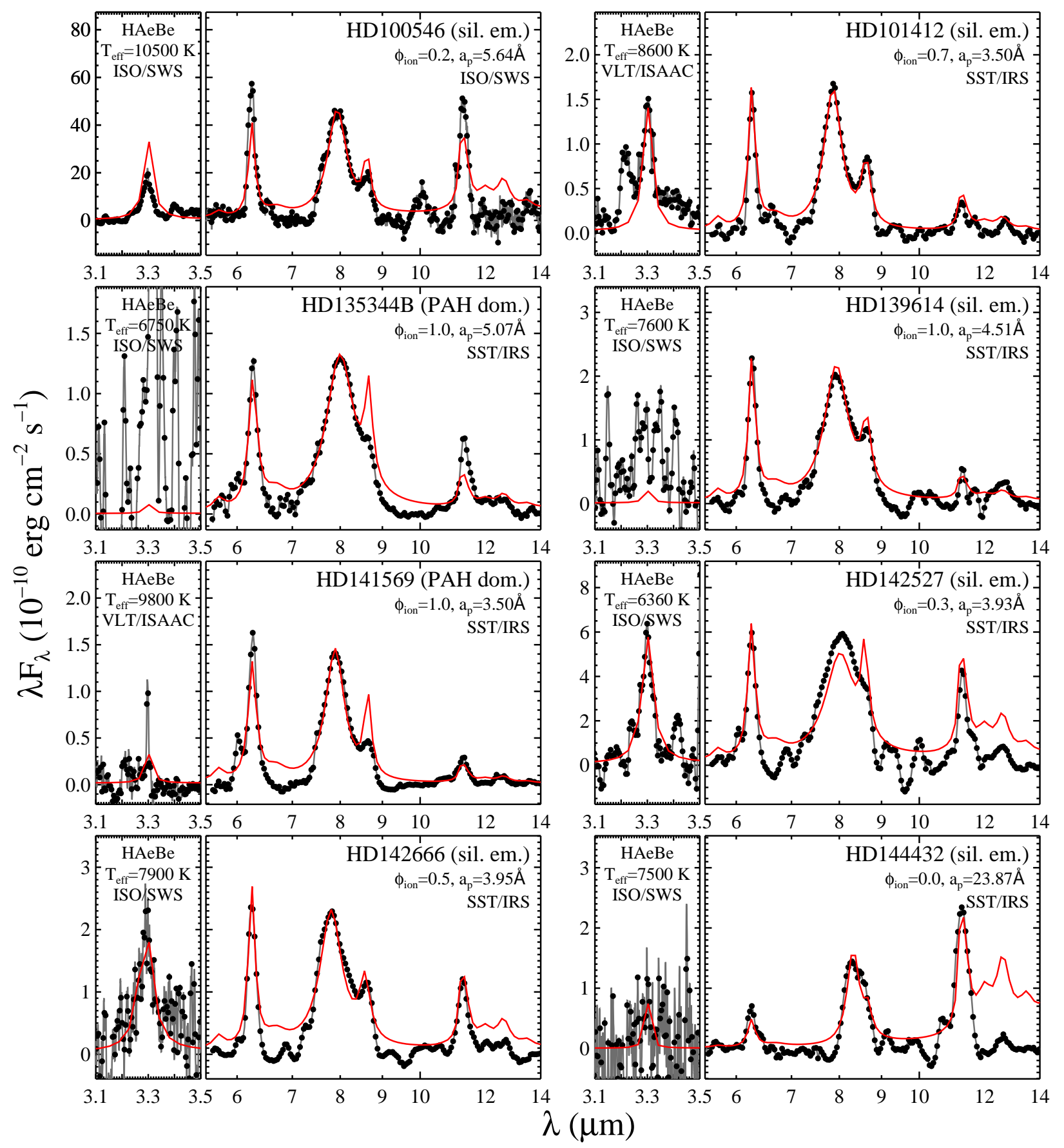

Fig. 4.- Same as Figure 1 but for HD 100546, HD 101412, HD 135344B, HD 139614, HD 141569, HD 142527, HD 142666, and HD 144432. 


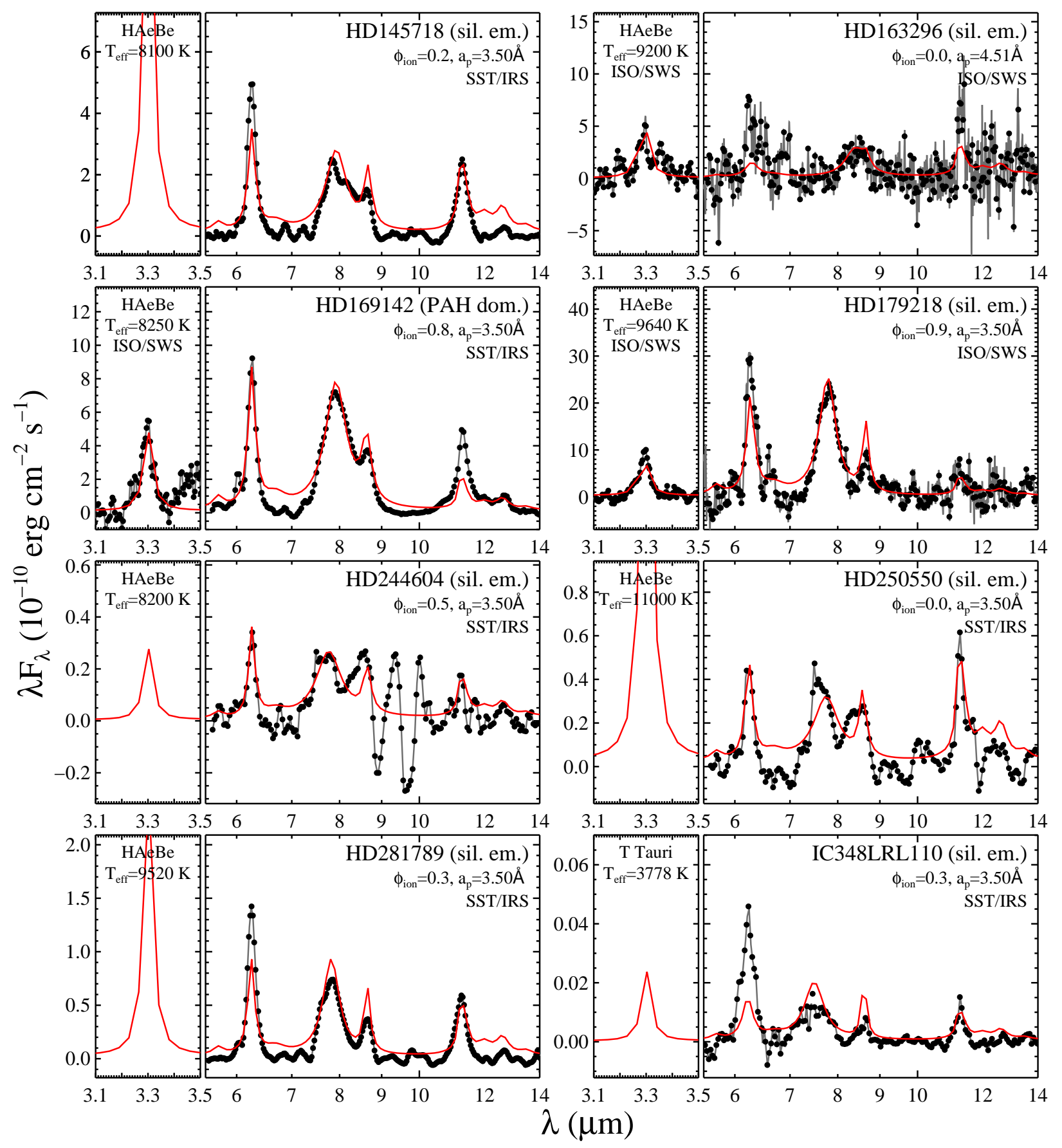

Fig. 5.- Same as Figure 1 but for HD 145718, HD 163296, HD 169142, HD 179218, HD 244604, HD 250550, HD 281789, and IC348 LRL110. 

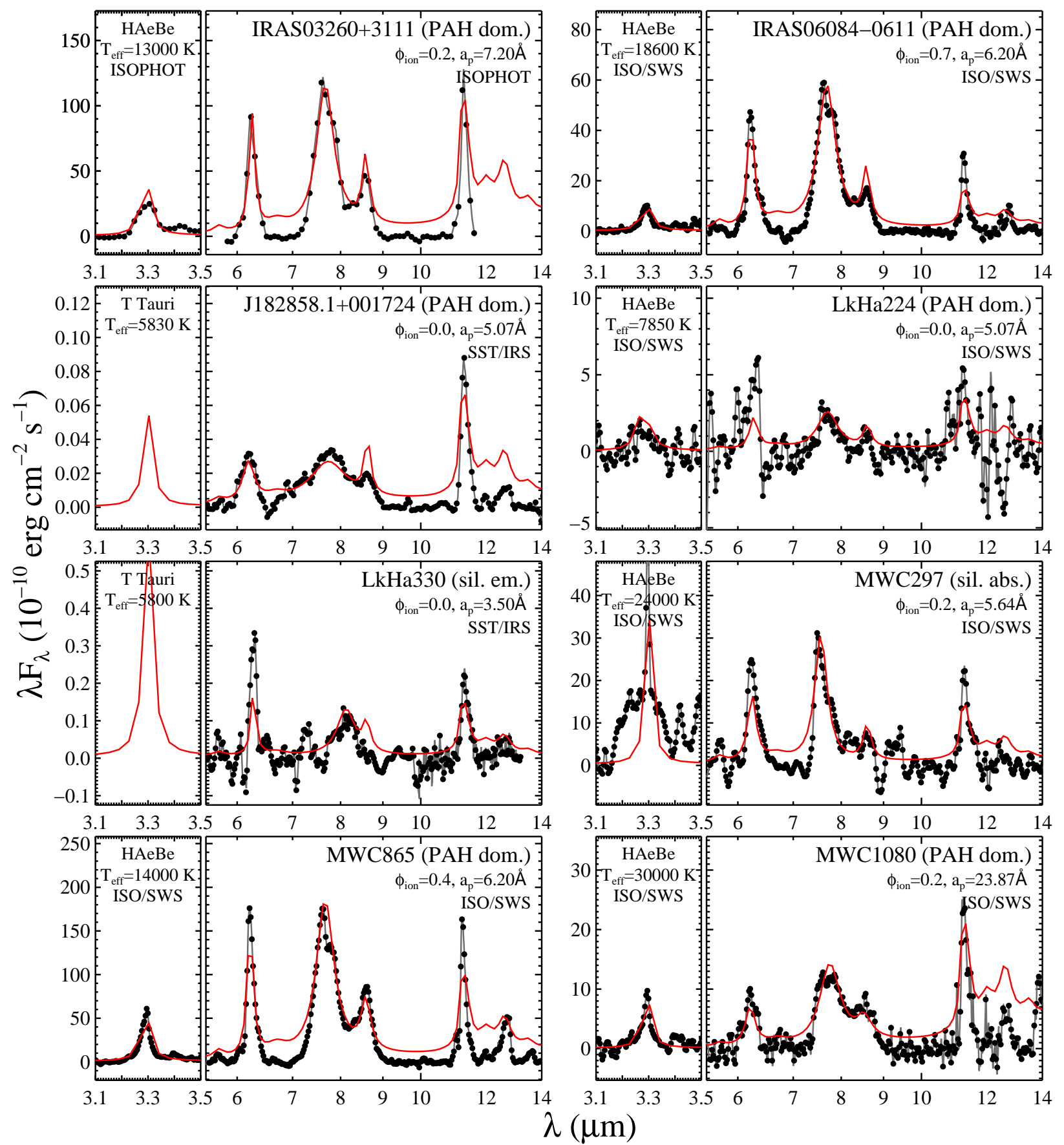

Fig. 6.- Same as Figure1 but for IRAS 03260+3111, IRAS 06084-0611, J182858.1+001724, LkH $\alpha$ 224, LkH $\alpha$ 330, MWC 297, MWC 865, and MWC 1080. 

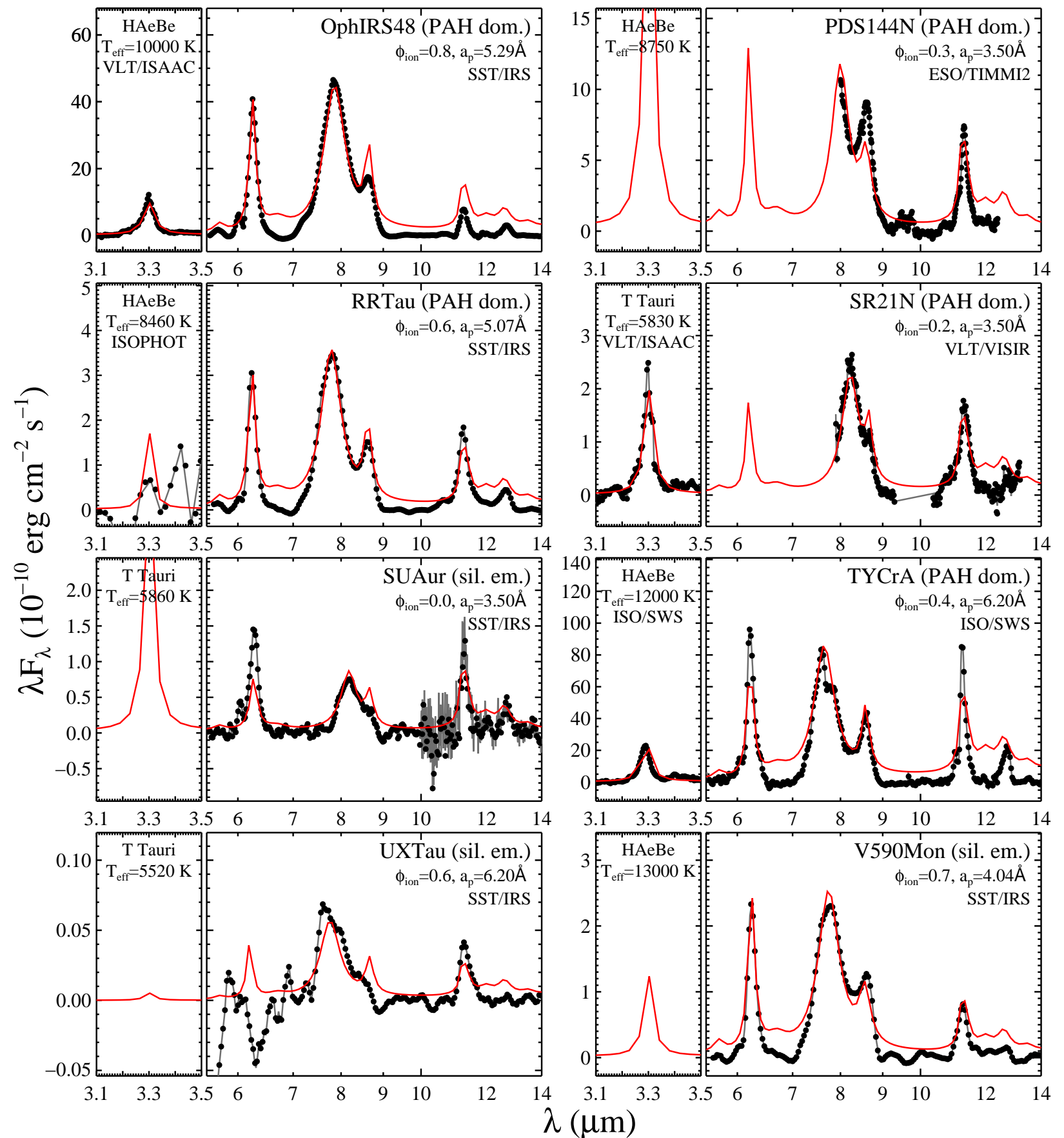

Fig. 7.- Same as Figure 1 but for Oph IRS48, PDS 144N, RR Tau, SR 21N, SU Aur, TY CrA, UX Tau, and V590 Mon. 

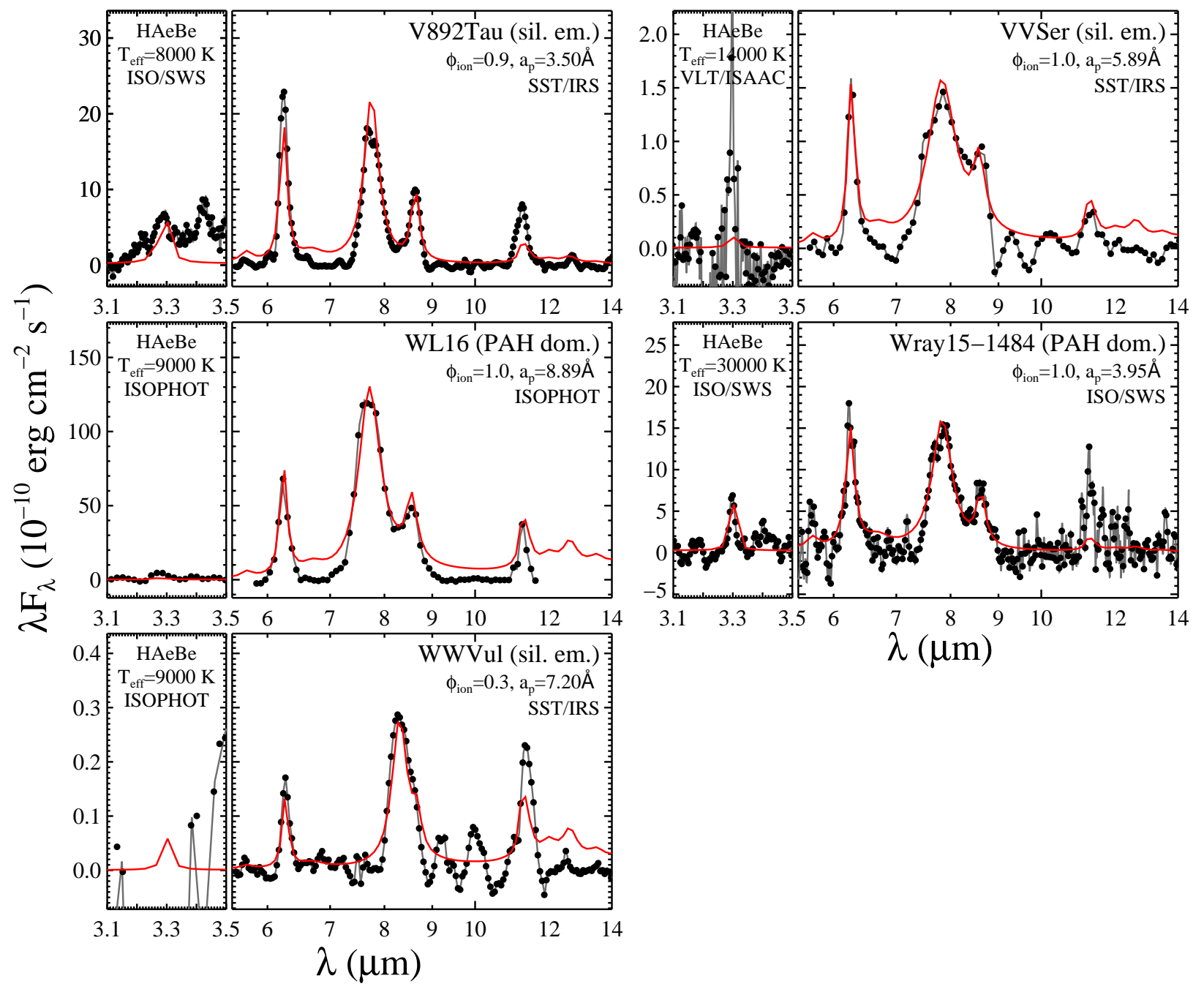

Fig. 8.- Same as Figure1 but for V892 Tau, VV Ser, WL 16, Wray 15-1484, and WW Vul. 


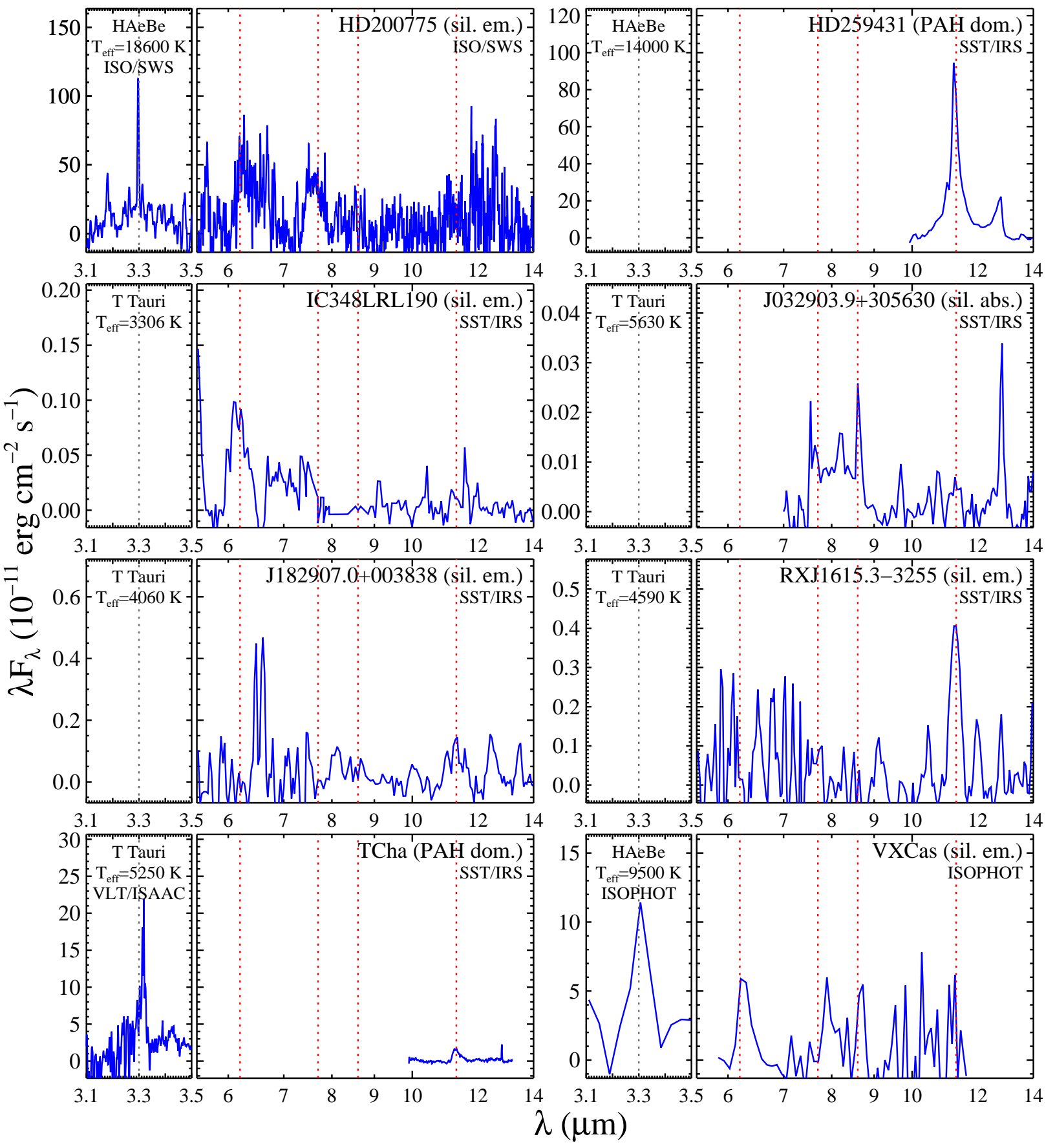

Fig. 9.- Residual spectra of the eight sources (blue lines) that are excluded for PAH modeling due to either a limited wavelength coverage or an insufficient $\mathrm{S} / \mathrm{N}$ (see Section 2.3). As a reference, the peak wavelengths of the major PAH features (i.e., 3.3, 6.2, 7.7, 8.6, and $11.2 \mu \mathrm{m}$ ) are marked (dotted lines). 
to survive in the diffuse interstellar medium (ISM; see Li \& Draine 2001b). Figure 10 shows the PAH size distribution expressed by multiplying $a^{4}$ to show the mass distribution of PAHs. For this distribution, $a^{4} d n / d a$ peaks at $a_{\mathrm{p}}=a_{0} \exp \left(3 \sigma^{2}\right)$ (Li \& Draine 2001a). Since $a^{4} d n / d a$ is truncated at $a_{\min }^{\mathrm{PAH}}=3.5 \AA$, we set $a_{\mathrm{p}}=a_{\min }^{\mathrm{PAH}}=3.5 \AA$ when $a_{0} \exp \left(3 \sigma^{2}\right)<a_{\min }^{\mathrm{PAH}}$.

We adopt the PAH absorption cross-sections that are expressed as a series of Drude profiles (Li \& Draine 2001b; Draine \& Li 2007). It has been noticed that the feature profiles of PAH emission vary from one object to another (e.g., Peeters et al. 2002; Sloan et al. 2007), and the PAH emission features seen in PPDs often show deviations from those of the diffuse ISM (e.g., Keller et al. 2008). For each source, the peak wavelengths and widths of some of the Drude profiles are slightly modified to be consistent with the observed spectrum. In this way, the observed residual spectra can be fitted more closely (e.g., see Seok \& Li 2015, 2016). Note that the integrated area of each Drude profile is kept unchanged so that the model calculations are coherent through the entire sample. We distinguish the absorption cross-sections of neutral PAHs from those of charged PAHs, but neither cations from anions nor multiply charged PAHs are considered separately. The absorption cross sections of PAH cations and anions are rather close (see Li \& Draine 2001b) since the IR properties of PAH anions closely resemble those of PAH cations except for the very strong $3.3 \mu \mathrm{m} \mathrm{C}-\mathrm{H}$ stretch enhancement in the anion (e.g., Szczepanski et al. 1995; Langhoff 1996; Hudgins et al. 2000).

The charging of PAHs is determined by two competing mechanisms, photoionization and electron recombination (Bake \& Tielens 1994; Weingartner \& Draine 2001). We quantify the charge state of PAHs in our sample disks by using the ionization fraction $\left(\phi_{\text {ion }}\right)$, which is the probability of finding a PAH molecule in a nonzero charge state ( $\mathrm{Li} \&$ Lunine 2003). The ionization fraction $\phi_{\text {ion }}$ is determined by the PAH size (a), the UV starlight intensity $(U)$, the electron density $\left(n_{\mathrm{e}}\right)$, and the gas temperature $\left(T_{\text {gas }}\right)$. We define $U$ as the intensity of the stellar radiation between $912 \AA$ and $1 \mu \mathrm{m}$ with respect to that of the local interstellar radiation field (ISRF) of Mathis et al. (1983; MMP),

$$
U(r)=\frac{\left(R_{*} / 2 r\right)^{2} \int_{912 \AA}^{1 \mu \mathrm{m}} F_{\lambda}^{*} d \lambda}{\int_{912 \mathrm{~A}}^{1 \mu \mathrm{m}} c u_{\lambda}^{\mathrm{ISRF}} d \lambda},
$$

where $R_{*}$ is the stellar radius, $F_{\lambda}^{*}$ is the flux per unit wavelength (in units of $\operatorname{ergs~s}^{-1} \mathrm{~cm}^{-2} \mu \mathrm{m}^{-1}$ ) at the top of the central star's atmosphere, which is approximated by the Kurucz model atmospheric spectrum (Kurucz 1979), $c$ is the speed of the light, and $u_{\lambda}^{\mathrm{ISRF}}$ is the energy density of the MMP interstellar radiation field. To calculate $\phi_{\text {ion }}$ precisely, it is required to take into account the spatial (radial) variations of $U$ and $n_{\mathrm{e}}$, which are not always available in our sample. Thus, we assume a constant $\phi_{\text {ion }}$ for all PAH sizes, which can be a representative charge state of PAHs in the disk. 


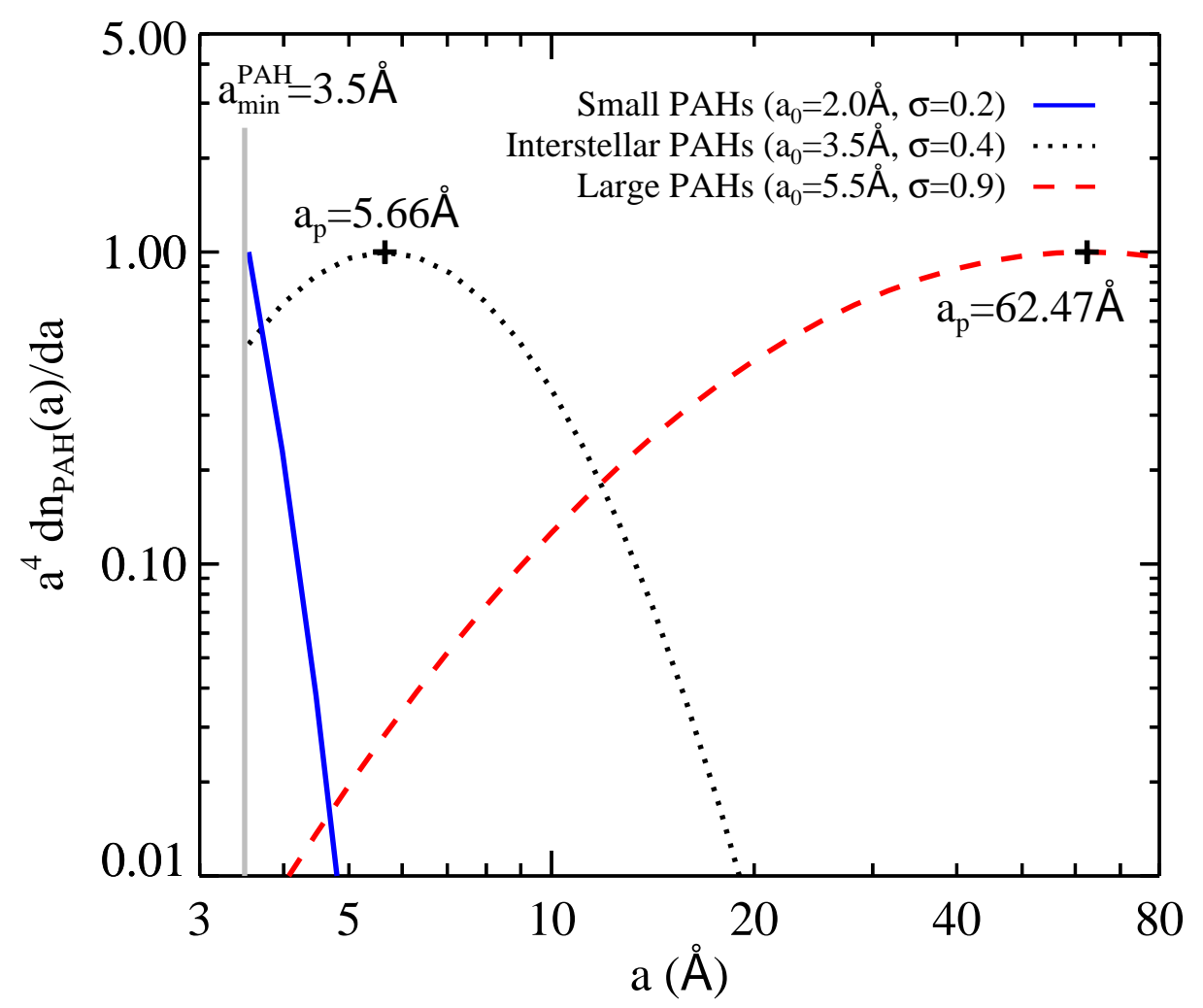

Fig. 10. - Log-normal size distribution $\left(d n_{\mathrm{PAH}} / d a\right)$ of PAHs, which is characterized by $a_{0}$ and $\sigma$ (Equation 1). We plot $a^{4} d n_{\mathrm{PAH}} / d a$ (normalized to its peak value) to show the mass distribution per logarithmic PAH radius, which peaks at $a_{\mathrm{p}}=a_{0} \exp \left(3 \sigma^{2}\right)$. For illustration, we show three different distributions: small PAHs $\left(a_{0}=2.0 \AA\right.$ and $\left.\sigma=0.2\right)$, PAHs in the diffuse ISM $\left(a_{0}=3.5 \AA\right.$ and $\sigma=0.4 ;$ Li \& Draine 2001b), and large PAHs $\left(a_{0}=5.5 \AA\right.$ and $\left.\sigma=0.9\right)$. We mark the peak sizes for the diffuse ISM PAH mass distribution $\left(a_{\mathrm{p}} \approx 5.66 \AA\right)$ and the distribution of the large PAHs $\left(a_{\mathrm{p}} \approx 62.47 \AA\right)$. For the distribution of the small PAHs, however, $a_{\mathrm{p}}$ is not calculated because the derived $a_{\mathrm{p}}(\approx 2.25 \AA)$ is smaller than $a_{\min }^{\mathrm{PAH}}(=3.5 \AA)$, and we set $a_{\mathrm{p}}=a_{\mathrm{min}}^{\mathrm{PAH}}$ in such a case. 
Because of their small heat contents which are often smaller than the energy of a single stellar UV photon, PAHs will not acquire an equilibrium temperature, and instead, they will undergo transient heating and experience temperature fluctuations (see Li 2004). Because of this, we calculate the temperature probability distribution functions of PAHs of a given size $a$ and a given charge state (i.e., neutral or ionized) exposed to starlight of a given intensity $U$ and a given spectral shape. The spectral shape of the UV/optical starlight is approximated by the model atmospheric spectrum of Kurucz (1979), characterized by $T_{\text {eff }}$, the effective temperature of the star. For this purpose, we employ the "thermal-discrete" method which treats both heating (absorption of UV/optical stellar photons) and cooling (emission of IR photons) as discrete transitions, using a thermal approximation for the downward transition probabilities (Draine \& Li 2001). Then, we calculate the IR emissivity for a mixture of neutral and ionized PAHs with a log-normal size distribution. Since PAHs have the single-photon heating nature, the spectral appearance of PAH emission is independent of the starlight intensity $U$, so the absolute emissivity level simply scales with $U$ (Draine \& Li 2001; Li \& Draine 2001b). Therefore, the total mass of PAHs ( $\left.M_{\mathrm{PAH}}\right)$ required to account for the observed PAH emission bands in the disk is inversely proportional to $U$ (see Li \& Lunine 2003).

In summary, there are three key free parameters in modeling the residual spectra: $a_{0}$ and $\sigma$ for the PAH size distribution and $\phi_{\text {ion }}$ for the charge state. We vary $a_{0}$ from 2.0 to 5.5 $\AA$ with a step of $0.5 \AA, \sigma$ from 0.2 to 0.9 with a step of 0.1 , and $\phi_{\text {ion }}$ from 0 to 1 with a step of 0.1. For a given set of the PAH parameters, the total mass of PAHs $\left(M_{\mathrm{PAH}}\right)$ is determined by the absolute level of the observed PAH flux density. For each source, $704(=8 \times 8 \times 11)$ models are calculated according to the stellar parameters of each source (e.g., $L_{\star}$ and $T_{\text {eff }}$ listed in Table 1), and we search for the best-fit model that reproduces the residual spectrum most closely.

\section{Results}

Among the 704 model calculations of each source, we select the best-fit model based on the least- $\chi^{2} /$ dof value. The best-fit models of the 61 sources are shown with their residual spectra in Figures 118, and the PAH parameters $\left(a_{0}, \sigma\right.$, and $\left.\phi_{\text {ion }}\right)$ of the best-fit models are listed in Table 2. We also derive the peak of the PAH mass distribution (per logarithmic PAH radius), $a_{\mathrm{p}}$, from $a_{0}$ and $\sigma$ (see Figure 10 and Section 3). The peak size $a_{\mathrm{p}}$ is beneficial to describe and compare the overall PAH size distributions since $a_{0}$ or $\sigma$ cannot solely represent the log-normal size distribution. Instead, the peak size is determined by the combination of these two parameters. 
In addition to the best-fit model, we select the top 70 models of each source (i.e., $\sim 10 \%$ of the total 704 model calculations), which in fact reproduce the residual spectra reasonably well, and calculate the median value (e.g., $\left\langle a_{\mathrm{p}}\right\rangle$ and $\left.\left\langle\phi_{\text {ion }}\right\rangle\right)$ and the median absolute deviation (MAD; e.g., $\Delta a_{\mathrm{p}}$ and $\Delta \phi_{\text {ion }}$ ) for each parameter (see Table 2). The MAD is defined as $\Delta X=\left\langle\left|X_{i}-\left\langle X_{i}\right\rangle\right|\right\rangle$, where $X_{i}$ is a univariate data set. The MAD is less sensitive to the outliers in a data set than the standard deviation, so it is considered as a robust estimator of the spread in the top 70 models for each parameter. We adopt the MAD of the top 70 models as uncertainties for each parameter. By taking the median and MAD for each parameter, we evaluate the robustness of the $\mathrm{PAH}$ parameters and look for the correlation of PAH properties with stellar parameters.

Finally, we examine the reliability of the model-fit parameters by taking into account both $\chi^{2} /$ dof and visual inspection. The reason why we perform the visual inspection supplementally is that, for some sources their $\chi^{2} /$ dof values may be low (which means that the best-fit model successfully reproduces the residual spectra), however, the residual spectra themselves, in particular of those sources with weak PAH features superimposed on the strong $9.7 \mu \mathrm{m}$ silicate emission feature, may not be very reliable, probably due to the imperfect continuum-subtraction (e.g., Acke et al. 2010). In these cases, the derivation of the PAH features at $\sim 7-9 \mu \mathrm{m}$ from the observed spectra is most likely to be affected, which is critical for constraining the model parameters. As a result, the best-fit parameters we derive might deviate from the true properties of the PAH molecules in the disks. Therefore, we shall distinguish these sources from those whose residual spectra are highly reliable for further analysis. We mainly use two criteria for determining the reliabilities of the model fits: (i) $\chi^{2} /$ dof $\lesssim 3.0$, and (ii) the absence of any appreciable extra residual in the observed spectrum except the PAH features after subtracting the $9.7 \mu \mathrm{m}$ silicate feature and the dust thermal emission continuum from the observed spectrum. It turns out that, for most of the sources which are classified as with low reliabilities, their $\chi^{2} /$ dof values are greater than 3.0; but for some sources, even though they have low $\chi^{2} /$ dof values (e.g., HD 37806, HD 58647), they are still classified as with low reliabilities due to the presence of extra residual, in addition to the PAH features, in the observed spectra after subtracting the dust thermal continuum emission and the $9.7 \mu \mathrm{m}$ silicate feature. We note that there are also some exceptions due to an incomplete wavelength coverage of the data sets (e.g., HD 98922 and PDS 144N are considered as having a low reliability since their data are only available at $\lambda \gtrsim 8 \mu \mathrm{m}$, in spite of their low $\chi^{2} /$ dof values and the absence of any extra residual in the their subtracted spectra). The reliability of each source is listed in Table 2,

We classify the sample targets into two groups: PAH-dominated spectra ("PAH dom.") and those with a strong silicate emission ("sil. em.") or absorption ("sil. abs") feature at $9.7 \mu \mathrm{m}$. When the PAH parameters are compared with the stellar parameters, we examine 
if the two groups show a different trend and calculate the correlation coefficients both separately and combined (see Figure 11 14). Note that we only consider those sources with reliable, high $\mathrm{S} / \mathrm{N}$ residual spectra when we examine the correlations.

Using the best-fit model, we examine the total mass of the PAHs $\left(M_{\mathrm{PAH}}\right)$ in PPDs. As $M_{\mathrm{PAH}}$ depends on the starlight intensity $(U)$, which is a function of distance, $r$, from the central star (i.e., $M_{\mathrm{PAH}} \propto 1 / U$ and $U \propto 1 / r^{2}$ ), we cannot constrain $M_{\mathrm{PAH}}$ exactly unless the spatial distribution of PAHs in the disk is properly taken into account. Instead, we derive $M_{\mathrm{PAH}}$ at $10 \mathrm{AU}\left(M_{\mathrm{PAH}}^{10 \mathrm{AU}}\right)$ assuming that all PAHs are located at $10 \mathrm{AU}$ from the central star. Then, if the spatial distribution of PAHs is revealed, we can easily calculate $M_{\mathrm{PAH}}$ by scaling with $r$ (i.e., $\left.M_{\mathrm{PAH}}=M_{\mathrm{PAH}}^{10 \mathrm{AU}}[r / 10 \mathrm{AU}]^{2}\right) . M_{\mathrm{PAH}}^{10 \mathrm{AU}}$ of each disk is listed in Table 2 , 
Table 2. PAH Parameters of 61 PPDs as Derived from the Astro-PAH Model Calculations

\begin{tabular}{|c|c|c|c|c|c|c|c|c|c|c|}
\hline \multirow[t]{2}{*}{ Object } & \multicolumn{5}{|c|}{ Best-fit } & \multicolumn{4}{|c|}{ Median of Top 70 Models $^{\mathrm{a}}$} & \multirow[t]{2}{*}{ Reliability } \\
\hline & $\begin{array}{c}a_{0} \\
(\AA)\end{array}$ & $\sigma$ & $\begin{array}{c}a_{\mathrm{p}} \\
(\AA)\end{array}$ & $\phi_{\text {ion }}$ & $\begin{array}{c}M_{\mathrm{PAH}}^{10 \mathrm{AUc}} \\
\left(10^{-6} M_{\oplus}\right)\end{array}$ & $\begin{array}{l}\left\langle a_{0}\right\rangle \\
(\AA)\end{array}$ & $\langle\sigma\rangle$ & $\begin{array}{l}\left\langle a_{\mathrm{p}}\right\rangle \\
(\AA)\end{array}$ & $\left\langle\phi_{\text {ion }}\right\rangle$ & \\
\hline AB Aur & 5.0 & 0.2 & 5.64 & 0.7 & 1.96 & $4.0 \pm 1.0$ & $0.4 \pm 0.1$ & $5.64 \pm 0.56$ & $0.8 \pm 0.1$ & $\mathrm{~h}$ \\
\hline AK Sco & 2.5 & 0.3 & 3.50 & 0.0 & 0.56 & $3.0 \pm 0.5$ & $0.3 \pm 0.1$ & $3.95 \pm 0.45$ & $0.1 \pm 0.1$ & 1 \\
\hline $\mathrm{BD}+40^{\circ} 4124$ & 5.5 & 0.4 & 8.89 & 0.3 & 1.01 & $4.0 \pm 1.0$ & $0.6 \pm 0.1$ & $10.59 \pm 3.18$ & $0.4 \pm 0.1$ & $\mathrm{~h}$ \\
\hline BF Ori & 2.5 & 0.6 & 7.36 & 0.1 & 0.44 & $4.0 \pm 1.0$ & $0.5 \pm 0.1$ & $8.08 \pm 1.73$ & $0.1 \pm 0.1$ & 1 \\
\hline DoAr21 & 5.5 & 0.2 & 6.20 & 0.1 & 12.4 & $4.0 \pm 1.0$ & $0.6 \pm 0.1$ & $10.31 \pm 2.94$ & $0.3 \pm 0.1$ & 1 \\
\hline EC82 & 2.0 & 0.2 & 3.50 & 0.0 & 540 & $2.5 \pm 0.5$ & $0.2 \pm 0.0$ & $3.50 \pm 0.00$ & $0.2 \pm 0.2$ & 1 \\
\hline HD 31648 & 4.5 & 0.2 & 5.07 & 0.4 & 1.56 & $3.5 \pm 1.0$ & $0.3 \pm 0.1$ & $5.07 \pm 0.82$ & $0.5 \pm 0.1$ & 1 \\
\hline HD 34282 & 4.0 & 0.2 & 4.51 & 0.8 & 3.86 & $3.5 \pm 1.0$ & $0.3 \pm 0.1$ & $4.58 \pm 0.65$ & $0.8 \pm 0.1$ & $\mathrm{~h}$ \\
\hline HD 34700 & 2.0 & 0.2 & 3.50 & 0.5 & 17.7 & $2.5 \pm 0.5$ & $0.3 \pm 0.1$ & $3.93 \pm 0.43$ & $0.2 \pm 0.1$ & $\mathrm{~h}$ \\
\hline HD 35187 & 2.5 & 0.5 & 5.29 & 1.0 & 1.12 & $3.5 \pm 1.0$ & $0.4 \pm 0.1$ & $5.89 \pm 0.66$ & $0.9 \pm 0.1$ & $\mathrm{~h}$ \\
\hline HD 36112 & 5.0 & 0.2 & 5.64 & 0.3 & 0.96 & $4.0 \pm 1.0$ & $0.3 \pm 0.1$ & $5.29 \pm 0.60$ & $0.3 \pm 0.1$ & 1 \\
\hline HD 36917 & 5.5 & 0.3 & 7.20 & 0.3 & 0.62 & $4.0 \pm 1.0$ & $0.5 \pm 0.1$ & $7.41 \pm 1.42$ & $0.3 \pm 0.1$ & $\mathrm{~h}$ \\
\hline HD 37357 & 2.0 & 0.2 & 3.50 & 0.1 & 0.69 & $3.0 \pm 0.5$ & $0.3 \pm 0.1$ & $3.93 \pm 0.43$ & $0.2 \pm 0.1$ & l \\
\hline HD 37411 & 3.5 & 0.3 & 4.58 & 1.0 & 2.27 & $3.5 \pm 1.0$ & $0.3 \pm 0.1$ & $4.58 \pm 0.65$ & $0.9 \pm 0.1$ & $\mathrm{~h}$ \\
\hline HD 37806 & 5.0 & 0.5 & 10.59 & 0.6 & 0.41 & $3.5 \pm 1.0$ & $0.7 \pm 0.1$ & $13.64 \pm 3.34$ & $0.7 \pm 0.1$ & l \\
\hline HD 38120 & 2.0 & 0.2 & 3.50 & 0.3 & 0.96 & $2.5 \pm 0.5$ & $0.2 \pm 0.0$ & $3.50 \pm 0.00$ & $0.4 \pm 0.2$ & 1 \\
\hline HD 58647 & 5.5 & 0.2 & 6.20 & 1.0 & 0.052 & $4.0 \pm 1.0$ & $0.4 \pm 0.1$ & $6.55 \pm 1.26$ & $0.9 \pm 0.1$ & l \\
\hline HD 72106 & 2.0 & 0.2 & 3.50 & 0.5 & 2.87 & $2.5 \pm 0.5$ & $0.2 \pm 0.0$ & $3.50 \pm 0.00$ & $0.6 \pm 0.2$ & $\mathrm{~h}$ \\
\hline HD 85567 & 5.0 & 0.3 & 6.55 & 0.9 & 0.054 & $4.0 \pm 1.0$ & $0.4 \pm 0.1$ & $5.89 \pm 0.60$ & $0.9 \pm 0.1$ & $\mathrm{~h}$ \\
\hline HD 95881 & 4.0 & 0.2 & 4.51 & 0.7 & 4.32 & $3.0 \pm 1.0$ & $0.3 \pm 0.1$ & $4.23 \pm 0.73$ & $0.8 \pm 0.1$ & $\mathrm{~h}$ \\
\hline HD 97048 & 5.0 & 0.2 & 5.64 & 0.4 & 6.42 & $4.0 \pm 1.0$ & $0.4 \pm 0.1$ & $5.89 \pm 0.65$ & $0.5 \pm 0.1$ & $\mathrm{~h}$ \\
\hline HD 97300 & 5.5 & 0.2 & 6.20 & 0.9 & 1.60 & $4.0 \pm 1.0$ & $0.4 \pm 0.1$ & $5.89 \pm 0.65$ & $0.9 \pm 0.1$ & $\mathrm{~h}$ \\
\hline HD 98922 & 2.5 & 0.2 & 3.50 & 0.6 & 0.99 & $3.0 \pm 0.5$ & $0.3 \pm 0.1$ & $3.95 \pm 0.45$ & $0.5 \pm 0.1$ & 1 \\
\hline HD 100453 & 2.0 & 0.2 & 3.50 & 0.7 & 4.72 & $3.0 \pm 0.5$ & $0.3 \pm 0.1$ & $4.04 \pm 0.54$ & $0.4 \pm 0.2$ & $\mathrm{~h}$ \\
\hline HD 100546 & 5.0 & 0.2 & 5.64 & 0.2 & 5.37 & $3.5 \pm 1.0$ & $0.3 \pm 0.1$ & $5.07 \pm 0.82$ & $0.5 \pm 0.2$ & $\mathrm{~h}$ \\
\hline HD 101412 & 2.0 & 0.2 & 3.50 & 0.7 & 3.13 & $3.0 \pm 0.5$ & $0.3 \pm 0.1$ & $3.95 \pm 0.45$ & $0.5 \pm 0.1$ & $\mathrm{~h}$ \\
\hline HD 135344B & 4.5 & 0.2 & 5.07 & 1.0 & 1.55 & $3.5 \pm 1.0$ & $0.3 \pm 0.1$ & $5.07 \pm 1.03$ & $0.9 \pm 0.1$ & $\mathrm{~h}$ \\
\hline
\end{tabular}


Table 2-Continued

\begin{tabular}{|c|c|c|c|c|c|c|c|c|c|c|}
\hline \multirow[t]{2}{*}{ Object } & \multicolumn{5}{|c|}{ Best-fit } & \multicolumn{4}{|c|}{ Median of Top 70 Models $^{\mathrm{a}}$} & \multirow[t]{2}{*}{ Reliability $^{\mathrm{b}}$} \\
\hline & $\begin{array}{l}a_{0} \\
(\AA)\end{array}$ & $\sigma$ & $\begin{array}{c}a_{\mathrm{p}} \\
(\AA)\end{array}$ & $\phi_{\text {ion }}$ & $\begin{array}{c}M_{\mathrm{PAH}}^{10 \mathrm{AUc}} \\
\left(10^{-6} M_{\oplus}\right)\end{array}$ & $\begin{array}{l}\left\langle a_{0}\right\rangle \\
(\AA)\end{array}$ & $\langle\sigma\rangle$ & $\begin{array}{l}\left\langle a_{\mathrm{p}}\right\rangle \\
(\AA)\end{array}$ & $\left\langle\phi_{\text {ion }}\right\rangle$ & \\
\hline HD 139614 & 4.0 & 0.2 & 4.51 & 1.0 & 1.83 & $3.0 \pm 1.0$ & $0.3 \pm 0.1$ & $4.23 \pm 0.73$ & $0.8 \pm 0.1$ & $\mathrm{~h}$ \\
\hline HD 141569 & 2.0 & 0.2 & 3.50 & 1.0 & 0.19 & $3.0 \pm 1.0$ & $0.3 \pm 0.1$ & $4.51 \pm 0.73$ & $0.9 \pm 0.1$ & $\mathrm{~h}$ \\
\hline HD 142527 & 3.0 & 0.3 & 3.93 & 0.3 & 7.51 & $3.0 \pm 1.0$ & $0.3 \pm 0.1$ & $4.23 \pm 0.73$ & $0.3 \pm 0.1$ & $\mathrm{~h}$ \\
\hline HD 142666 & 3.5 & 0.2 & 3.95 & 0.5 & 2.08 & $3.0 \pm 0.5$ & $0.3 \pm 0.1$ & $4.04 \pm 0.54$ & $0.5 \pm 0.1$ & $\mathrm{~h}$ \\
\hline HD 144432 & 3.5 & 0.8 & 23.87 & 0.0 & 6.50 & $4.5 \pm 1.0$ & $0.7 \pm 0.1$ & $19.57 \pm 8.70$ & $0.0 \pm 0.0$ & 1 \\
\hline HD 145718 & 2.0 & 0.2 & 3.50 & 0.2 & 5.17 & $2.5 \pm 0.5$ & $0.2 \pm 0.0$ & $3.50 \pm 0.00$ & $0.4 \pm 0.2$ & 1 \\
\hline HD 163296 & 4.0 & 0.2 & 4.51 & 0.0 & 0.80 & $3.0 \pm 0.5$ & $0.3 \pm 0.1$ & $3.93 \pm 0.43$ & $0.2 \pm 0.2$ & 1 \\
\hline HD 169142 & 2.5 & 0.2 & 3.50 & 0.8 & 9.06 & $3.0 \pm 0.5$ & $0.3 \pm 0.1$ & $4.58 \pm 1.05$ & $0.5 \pm 0.2$ & $\mathrm{~h}$ \\
\hline HD 179218 & 2.0 & 0.2 & 3.50 & 0.9 & 2.58 & $3.0 \pm 0.5$ & $0.3 \pm 0.1$ & $3.95 \pm 0.45$ & $0.7 \pm 0.1$ & $\mathrm{~h}$ \\
\hline HD 244604 & 2.0 & 0.4 & 3.50 & 0.5 & 0.44 & $3.0 \pm 0.5$ & $0.3 \pm 0.1$ & $4.04 \pm 0.54$ & $0.5 \pm 0.1$ & 1 \\
\hline HD 250550 & 2.0 & 0.2 & 3.50 & 0.0 & 0.98 & $3.0 \pm 0.5$ & $0.3 \pm 0.1$ & $4.23 \pm 0.73$ & $0.2 \pm 0.1$ & 1 \\
\hline HD 281789 & 2.0 & 0.2 & 3.50 & 0.3 & 5.60 & $2.5 \pm 0.5$ & $0.2 \pm 0.0$ & $3.50 \pm 0.00$ & $0.4 \pm 0.2$ & 1 \\
\hline IC348LRL110 & 2.0 & 0.2 & 3.50 & 0.3 & 82.7 & $2.5 \pm 0.5$ & $0.2 \pm 0.0$ & $3.50 \pm 0.00$ & $0.6 \pm 0.2$ & 1 \\
\hline IRAS $03260+3111$ & 5.5 & 0.3 & 7.20 & 0.2 & 23.0 & $4.0 \pm 1.0$ & $0.5 \pm 0.1$ & $8.89 \pm 1.98$ & $0.3 \pm 0.1$ & $\mathrm{~h}$ \\
\hline IRAS 06084-0611 & 5.5 & 0.2 & 6.20 & 0.7 & 7.91 & $3.5 \pm 1.0$ & $0.4 \pm 0.1$ & $6.35 \pm 1.11$ & $0.8 \pm 0.1$ & $\mathrm{~h}$ \\
\hline $\mathrm{J} 182858.1+001724$ & 4.5 & 0.2 & 5.07 & 0.0 & 2.20 & $3.5 \pm 1.0$ & $0.4 \pm 0.1$ & $5.89 \pm 1.30$ & $0.1 \pm 0.1$ & $\mathrm{~h}$ \\
\hline $\mathrm{LkH} \alpha 224$ & 4.5 & 0.2 & 5.07 & 0.0 & 25.2 & $3.0 \pm 1.0$ & $0.3 \pm 0.1$ & $4.23 \pm 0.73$ & $0.2 \pm 0.1$ & 1 \\
\hline $\mathrm{LkH} \alpha 330$ & 2.0 & 0.2 & 3.50 & 0.0 & 3.34 & $2.5 \pm 0.5$ & $0.2 \pm 0.0$ & $3.50 \pm 0.00$ & $0.2 \pm 0.2$ & 1 \\
\hline MWC 297 & 5.0 & 0.2 & 5.64 & 0.2 & 0.023 & $3.5 \pm 1.0$ & $0.3 \pm 0.1$ & $5.24 \pm 1.11$ & $0.3 \pm 0.2$ & 1 \\
\hline MWC 865 & 5.5 & 0.2 & 6.20 & 0.4 & 5.61 & $3.5 \pm 1.0$ & $0.4 \pm 0.1$ & $6.46 \pm 1.62$ & $0.5 \pm 0.1$ & $\mathrm{~h}$ \\
\hline MWC 1080 & 3.5 & 0.8 & 23.87 & 0.2 & 0.40 & $4.0 \pm 1.0$ & $0.7 \pm 0.1$ & $19.57 \pm 5.93$ & $0.2 \pm 0.1$ & $\mathrm{~h}$ \\
\hline Oph IRS48 & 2.5 & 0.5 & 5.29 & 0.8 & 24.3 & $3.5 \pm 1.0$ & $0.5 \pm 0.1$ & $7.27 \pm 1.62$ & $0.7 \pm 0.1$ & $\mathrm{~h}$ \\
\hline PDS144N & 2.0 & 0.2 & 3.50 & 0.3 & 737 & $3.0 \pm 1.0$ & $0.3 \pm 0.1$ & $3.93 \pm 0.43$ & $0.4 \pm 0.2$ & 1 \\
\hline RR Tau & 4.5 & 0.2 & 5.07 & 0.6 & 19.7 & $3.5 \pm 1.0$ & $0.3 \pm 0.1$ & $4.85 \pm 0.61$ & $0.7 \pm 0.1$ & $\mathrm{~h}$ \\
\hline SR21N & 2.0 & 0.4 & 3.50 & 0.2 & 10.5 & $3.0 \pm 0.5$ & $0.3 \pm 0.1$ & $3.95 \pm 0.45$ & $0.2 \pm 0.1$ & $\mathrm{~h}$ \\
\hline SU Aur & 2.0 & 0.2 & 3.50 & 0.0 & 6.78 & $2.5 \pm 0.5$ & $0.2 \pm 0.0$ & $3.50 \pm 0.00$ & $0.3 \pm 0.2$ & 1 \\
\hline TY CrA & 5.5 & 0.2 & 6.20 & 0.4 & 6.94 & $3.5 \pm 1.0$ & $0.4 \pm 0.1$ & $6.46 \pm 1.62$ & $0.5 \pm 0.2$ & $\mathrm{~h}$ \\
\hline
\end{tabular}


Table 2-Continued

\begin{tabular}{|c|c|c|c|c|c|c|c|c|c|c|}
\hline \multirow[t]{2}{*}{ Object } & \multicolumn{5}{|c|}{ Best-fit } & \multicolumn{4}{|c|}{ Median of Top 70 Models $^{\mathrm{a}}$} & \multirow[t]{2}{*}{ Reliability } \\
\hline & $\begin{array}{c}a_{0} \\
(\AA)\end{array}$ & $\sigma$ & $\begin{array}{c}a_{\mathrm{p}} \\
(\AA)\end{array}$ & $\phi_{\text {ion }}$ & $\begin{array}{c}M_{\mathrm{PAH}}^{10 \mathrm{AUc}} \\
\left(10^{-6} M_{\oplus}\right)\end{array}$ & $\begin{array}{l}\left\langle a_{0}\right\rangle \\
(\AA)\end{array}$ & $\langle\sigma\rangle$ & $\begin{array}{l}\left\langle a_{\mathrm{p}}\right\rangle \\
(\AA)\end{array}$ & $\left\langle\phi_{\text {ion }}\right\rangle$ & \\
\hline UX Tau & 5.5 & 0.2 & 6.20 & 0.6 & 0.50 & $4.5 \pm 1.0$ & $0.5 \pm 0.1$ & $8.08 \pm 1.45$ & $0.7 \pm 0.1$ & 1 \\
\hline V590 Mon & 2.5 & 0.4 & 4.04 & 0.7 & 1.24 & $3.5 \pm 1.0$ & $0.3 \pm 0.1$ & $4.58 \pm 0.64$ & $0.8 \pm 0.1$ & $\mathrm{~h}$ \\
\hline V892 Tau & 2.0 & 0.2 & 3.50 & 0.9 & 12.2 & $3.0 \pm 0.5$ & $0.3 \pm 0.1$ & $3.95 \pm 0.45$ & $0.7 \pm 0.2$ & $\mathrm{~h}$ \\
\hline VV Ser & 2.0 & 0.6 & 5.89 & 1.0 & 0.08 & $4.0 \pm 1.0$ & $0.4 \pm 0.1$ & $6.35 \pm 0.85$ & $0.9 \pm 0.1$ & $\mathrm{~h}$ \\
\hline WL 16 & 5.5 & 0.4 & 8.89 & 1.0 & 3.45 & $4.0 \pm 1.0$ & $0.6 \pm 0.1$ & $9.53 \pm 2.16$ & $0.9 \pm 0.1$ & $\mathrm{~h}$ \\
\hline Wray15-1484 & 3.5 & 0.2 & 3.95 & 1.0 & 1.26 & $3.5 \pm 1.0$ & $0.5 \pm 0.2$ & $8.08 \pm 2.84$ & $0.8 \pm 0.1$ & $\mathrm{~h}$ \\
\hline WW Vul & 5.5 & 0.3 & 7.20 & 0.3 & 0.27 & $4.0 \pm 1.0$ & $0.5 \pm 0.1$ & $8.89 \pm 1.68$ & $0.4 \pm 0.1$ & 1 \\
\hline
\end{tabular}

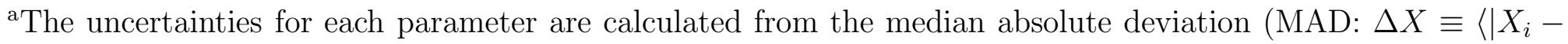
$\left\langle X_{i}\right\rangle|\rangle$, where $X_{i}$ is a univariate data set). The MAD is an estimator for each parameter of the spread in the top 70 models that closely fit the observed PAH spectrum (see Section 4).

${ }^{b}$ Reliability of the model-fit parameters (see Section 4). If the model-fit is highly reliable, we label it with "h". If the model-fit is less reliable, we label it "l". Note that only those with high reliability are used to investigate the correlations of the PAH properties

${ }^{\mathrm{c}}$ Total mass of PAHs under the assumption that all PAHs are located at $r=10 \mathrm{AU}$ from the central star. Total mass of PAHs at a given distance $r$ can be derived from $M_{\mathrm{PAH}}(r)=M_{\mathrm{PAH}}^{10 \mathrm{AU}}(r / 10 \mathrm{AU})^{2}$. 


\section{Discussion}

\subsection{Correlating PAH Properties with Stellar Parameters}

\subsubsection{Stellar Effective Temperature}

The effective temperature of the central star $\left(T_{\text {eff }}\right)$ is one of the key environmental parameters that control the physical and chemical properties of PAHs in a disk. In Figure 11, the peak size of the PAH size distribution $\left(a_{\mathrm{p}}\right)$ and the ionization fraction $\left(\phi_{\text {ion }}\right)$ are compared with $T_{\text {eff }}$. With a correlation coefficient of $R_{\mathrm{PAH}} \approx 0.77$, the PAH peak size $\left(a_{\mathrm{p}}\right)$ derived for those PAH-dominated sources seems to have a tendency of positively correlating with $T_{\text {eff }}$. Such a trend is relatively weak $\left(R_{\text {sil }} \approx 0.45\right)$ for those emitting or absorbing strongly the $9.7 \mu \mathrm{m}$ silicate feature. Combining both data sets, we obtain a correlation coefficient of $R_{\text {tot }} \approx 0.69$. We also derive the Kendall rank correlation coefficient $(\tau)$ and the significance of its deviation from zero $(p)$ for a non-parametric hypothesis test. In Kendall $\tau$ test, $p$ is in the interval between 0.0 and 1.0, and a smaller value indicates a more significant correlation. The Kendall test for the correlation between $a_{\mathrm{p}}$ and $T_{\text {eff }}$ gives $\tau \approx 0.57,0.26$, and 0.48 , and $p \approx 2.81 \times 10^{-4}, 0.25$, and $3.26 \times 10^{-5}$ for the PAH-dominated sources, the silicate-dominated sources, and the combined data sets, respectively. The low $p$ value for the PAH-dominated sources (and the combined data sets) indicates that the correlation between $a_{\mathrm{p}}$ and $T_{\text {eff }}$ is meaningful.

We perform a simple linear-fit in logarithmic scale, which gives $\left\langle a_{\mathrm{p}}\right\rangle=0.02_{-0.01}^{+0.04} \times$ $\left(T_{\text {eff }} / \mathrm{K}\right)^{0.6 \pm 0.1}, 0.37_{-0.25}^{+0.75} \times\left(T_{\text {eff }} / \mathrm{K}\right)^{0.3 \pm 0.1}$, and $0.11_{-0.06}^{+0.12} \times\left(T_{\text {eff }} / \mathrm{K}\right)^{0.4 \pm 0.1}$ for the PAH-dominated sources, the silicate-dominated sources, and the combined data sets, respectively. This trend can be readily explained: in those sources with a high $T_{\text {eff }}$, the stellar photons are more energetic, and therefore, the photodissociation of small PAHs is more likely to occur. In particular, small PAHs are more vulnerable than large ones because their internal vibrational modes are not large enough to quickly re-distribute the energy transferred from the absorbed stellar photons (see Section 5.3 for details). Thus, in disks around stars with a higher $T_{\text {eff }}$, small PAHs are preferentially photodissociated.

Unlike $a_{\mathrm{p}}, \phi_{\text {ion }}$ does not show any significant correlation with $T_{\text {eff }}$ (and other stellar parameters such as $L_{\star} / M_{\star}$ or stellar age, which are not shown here). From the Kendall test, we obtain $p \approx 0.54,0.10$, and 0.19 for the PAH-dominated sources, the silicate-dominated sources, and the combined data sets, respectively, which is much larger than those for $a_{\mathrm{p}}$. We note that $\phi_{\text {ion }}$ depends on $U \sqrt{T_{\text {gas }}} / n_{\mathrm{e}}$ (see Section 3) while $U$ is proportional to $r^{-2}$, so $\phi_{\text {ion }}$ depends on the distance of PAHs from the central star. Then, this lack of correlation between $\phi_{\text {ion }}$ and $T_{\text {eff }}$ could be attributed to the diversity of the spatial distribution of PAHs 
from one disk to another. In addition, $n_{\mathrm{e}}$ within a disk can span a wide range from a few to $\gtrsim 10^{5-6} \mathrm{~cm}^{-3}$ depending on the disk geometry, so the spatial distribution of PAHs should be taken into account to properly interpret $\phi_{\text {ion }}$. Note that there is also an environmental aspect to be considered. For example, in the case of MWC 1080, a member of a small star forming region, the diffuse nebulosity surrounding it is known to be the dominant origin of the PAH emission rather than its own disk (e.g., Li et al. 2014). Then, the PAH molecules in the nebula could be neutral despite of the rather high $T_{\text {eff }}(=30,000 \mathrm{~K})$ probably due to the highly diluted stellar radiation (i.e., low $U$ ) compared with that of a typical PPD.

\subsubsection{Luminosity-to-Mass Ratio}

In Figure 12, we compare $a_{\mathrm{p}}$ with $L_{\star} / M_{\star}$, the ratio of stellar luminosity to stellar mass. The correlation between $a_{\mathrm{p}}$ and $L_{\star} / M_{\star}$ appears as strong as that between $a_{\mathrm{p}}$ and $T_{\text {eff }}$ (for the PAH-dominated spectra, $R_{\mathrm{PAH}} \approx 0.80$ ). From the Kendall test, we derive $\tau \approx 0.44,0.30$, and 0.32 and $p \approx 0.01,0.11$, and 0.01 for the PAH-dominated sources, the silicate-dominated sources, and the combined data sets, respectively. Like $T_{\text {eff }}$, we perform a simple linear-fit in logarithmic scale, which gives $\left\langle a_{\mathrm{p}}\right\rangle=4.1_{-0.3}^{+0.3} \times\left(L_{\star} / M_{\star}\right)^{0.11 \pm 0.02}, 4.2_{-0.4}^{+0.4} \times\left(L_{\star} / M_{\star}\right)^{0.05 \pm 0.02}$, and $4.3_{-0.2}^{+0.2} \times\left(L_{\star} / M_{\star}\right)^{0.07 \pm 0.02}$ for the PAH-dominated sources, the silicate-dominated sources, and the combined data sets, respectively.

The luminosity-to-mass ratio can be a diagnostics of the relative strength of the radiative force against the gravitational force $\left(\beta_{\mathrm{RP}}\right.$, see more in Section 5.3). The radiation pressure blows materials away from the disk so that the size distribution of PAHs can be rearranged. However, as most of the sources in our sample are expected to possess a gas-rich disk, the gas drag would dominate the motion of PAHs rather than the radiation pressure. Then, the correlation we see in Figure 12 merely reflects the dependence of $L_{\star}$ on $T_{\text {eff }}$ (i.e., $L_{\star} \propto T_{\text {eff }}^{4}$ ). To investigate any influences of the radiation pressure, it is required to distinguish gas-rich and gas-depleted disks (see Section 5.3).

\subsubsection{Stellar Age}

We examine the correlation between $a_{\mathrm{p}}$ and the stellar age. As shown in Figure 13, $a_{\mathrm{p}}$ does not seem to exhibit any correlation with the stellar age, but the overall comparison might indicate a decreasing trend of $a_{\mathrm{p}}$ with the stellar age since all correlation coefficients (i.e., both $R$ and $\tau$ ) are negative (with Kendall's $\tau \approx-0.30$ and $p=0.14$ for the PAH dominated-spectra). If this is true, it would be interesting because small PAHs are more 

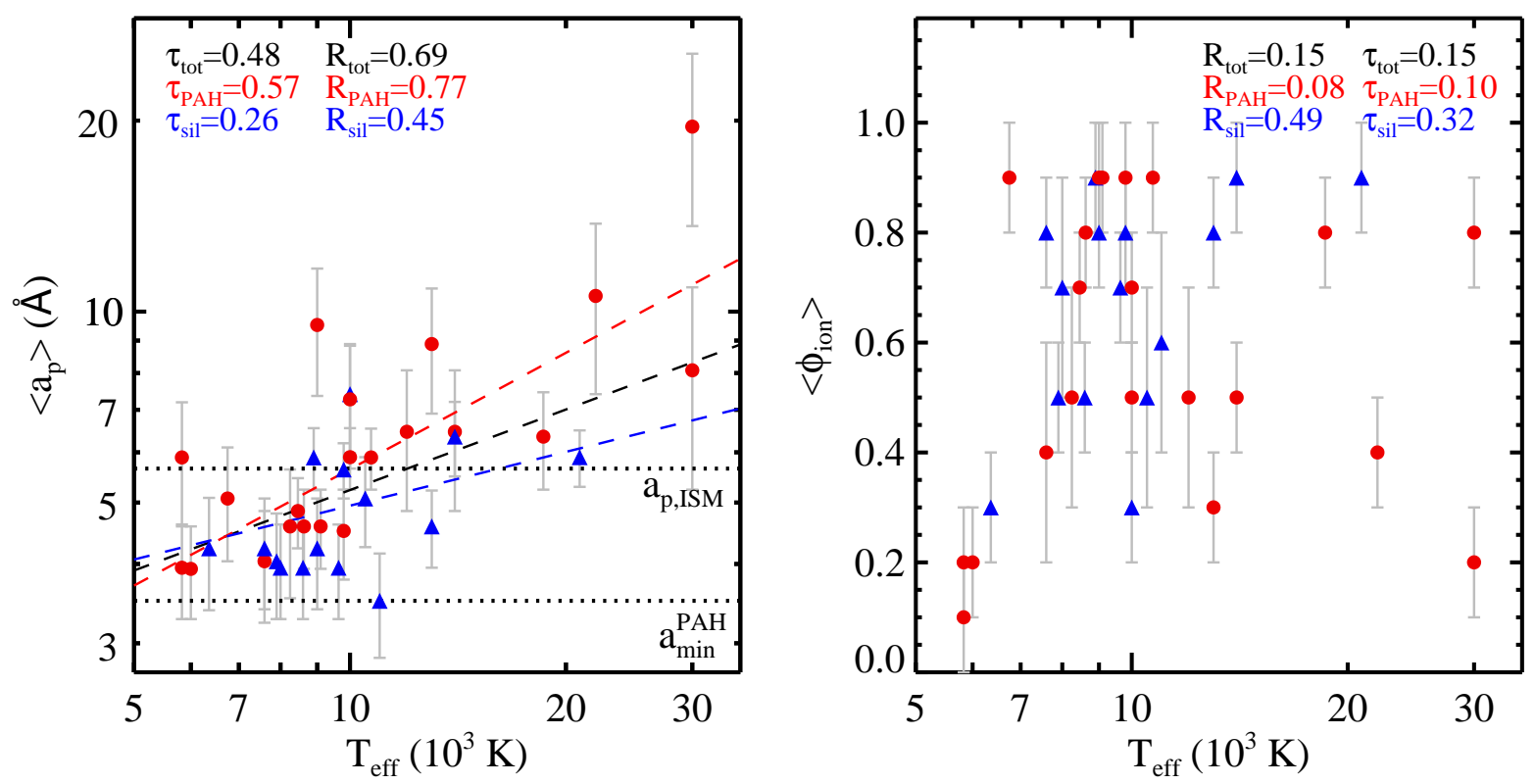

Fig. 11. - Comparison between the PAH parameters $\left(a_{\mathrm{p}}\right.$ and $\phi_{\text {ion }}$ in the left and right panels, respectively) and the stellar effective temperatures $\left(T_{\text {eff }}\right) .\left\langle a_{\mathrm{p}}\right\rangle$ and $\left\langle\phi_{\text {ion }}\right\rangle$ are median values in the top 70 models of each source, and uncertainties are the MAD (i.e., $\Delta a_{\mathrm{p}}$ and $\Delta \phi_{\text {ion }}$, see Section 4 and Table 2). Circle and triangle symbols denote sources with PAH emissiondominated and silicate emission (or absorption) dominated spectra, respectively. Note that we only consider those sources with the high reliability of the model-fit parameters (see Section 4 and Table 2). In the left panel, $a_{\mathrm{p}}$ for the diffuse ISM (i.e., $a_{\mathrm{p}, \mathrm{ISM}}=5.66 \AA$ ) and $a_{\mathrm{min}}^{\mathrm{PAH}}$ $\left(3.5 \AA\right.$, the minimum size of $a_{\mathrm{p}}$, see Section 3 ) are denoted by dotted lines as a reference. The correlations between $a_{\mathrm{p}}$ and $T_{\text {eff }}$ are fitted with a linear function in logarithmic scale (red, blue, and black dashed lines for the PAH-dominated sources, the silicate-dominated sources, and combined data sets, respectively). Correlation coefficients for the PAH-dominated spec$\operatorname{tra}\left(R_{\mathrm{PAH}}\right)$ and those with the strong $9.7 \mu \mathrm{m}$ silicate emission (or absorption) feature $\left(R_{\text {sil }}\right)$ are measured separately, and a total correlation coefficient is also given $\left(R_{\text {tot }}\right)$. Hereafter, symbol designation is applied in the same way for Figures $12,14$. 


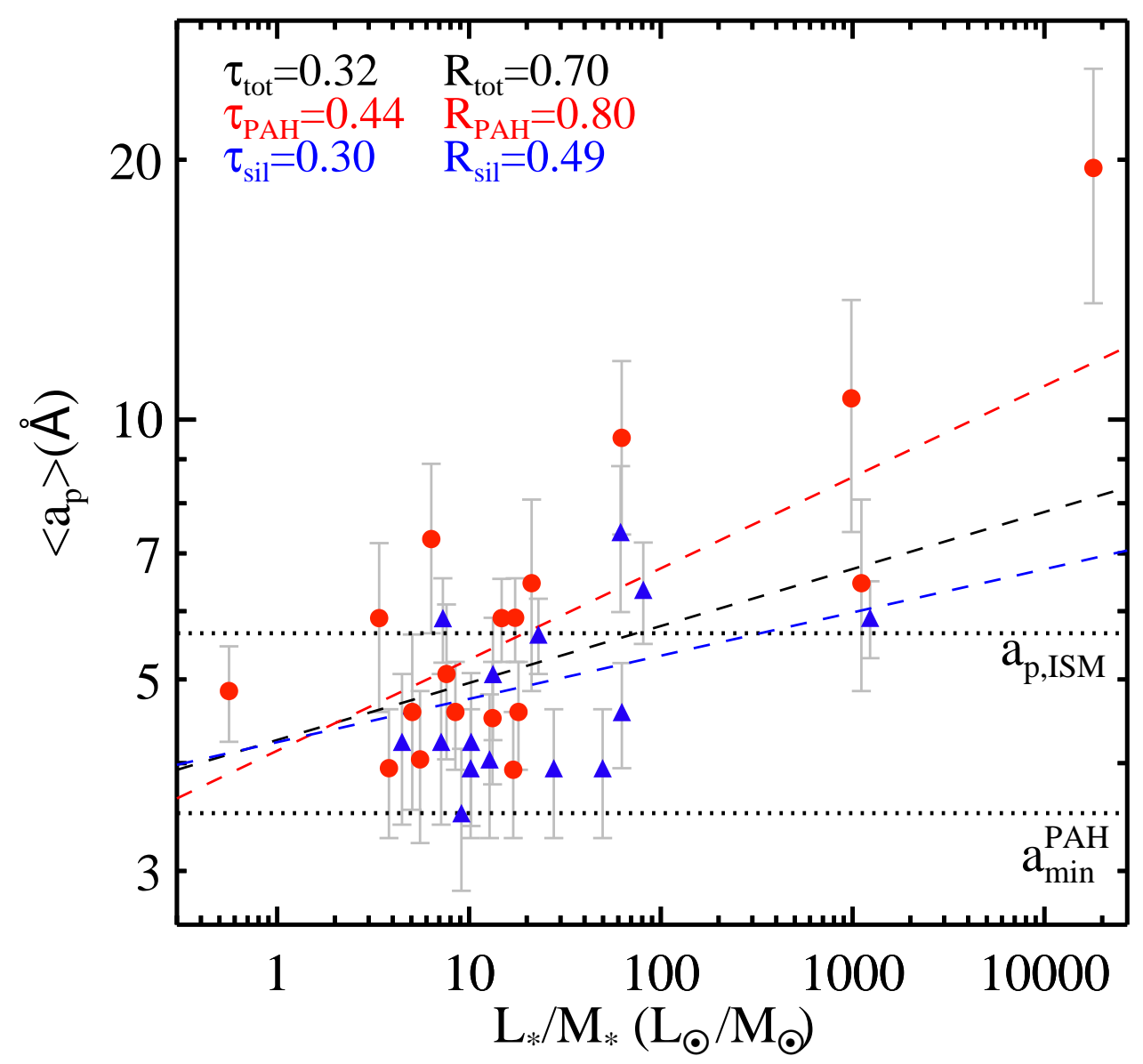

Fig. 12.- Comparison between $a_{\mathrm{p}}$ and $L_{\star} / M_{\star}$. 


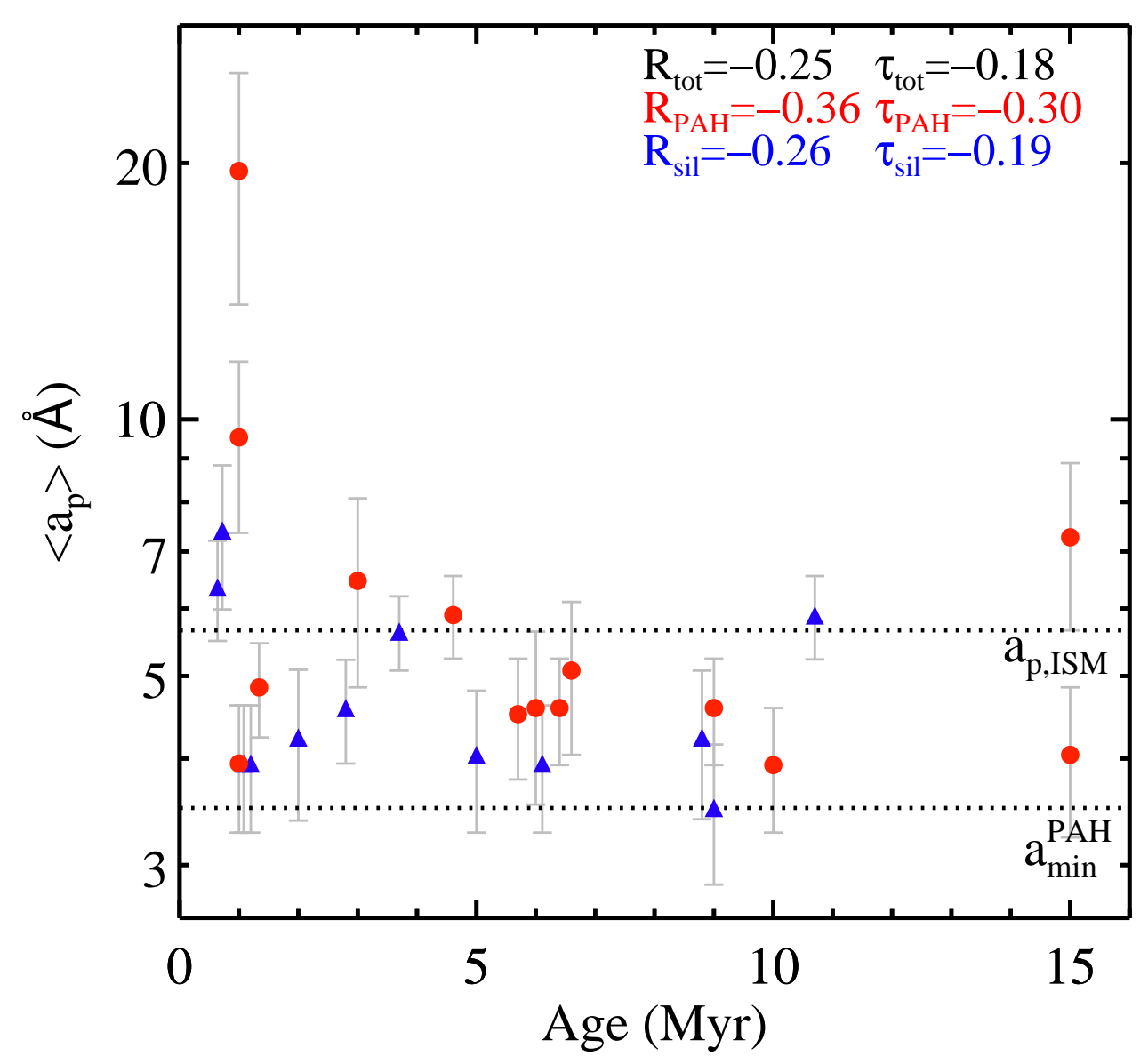

Fig. 13.- Comparison between $a_{\mathrm{p}}$ and stellar age.

quickly destroyed by photodissociation than large PAHs (see Section 5.3 and Figure 16). To maintain the abundance of small PAHs, it is required that small PAHs are rapidly replenished unless the photodissociation is not effective due to very mild environment such as low $T_{\text {eff }}$ and low $L_{\star}$ (e.g., IC348 LRL110: $T_{\text {eff }}=3778 \mathrm{~K}$ and $L_{\star}=0.22 L_{\odot}$ ). If fragmentation is a primary mechanism of the replenishment, the existence of small PAH molecules in aged disks could be explained.

Note that the estimation of stellar age often has large uncertainties and is modeldependent. Also, for some sources, their ages have not been estimated mostly due to the poorly constrained distance to the sources. For this reason, fewer sources are used to find a correlation compared with other cases. Once Gaia, a space observatory of ESA, dedicated 
to astrometry releases the data 5 we expect that the accuracy of age estimation would be much improved, and the correlation with the stellar age can be revisited.

\subsection{The $7.7 \mu \mathrm{m}$ feature}

The PAH feature at $7.7 \mu \mathrm{m}$ attributed to the $\mathrm{C}-\mathrm{C}$ stretching mode has been known to show large variation in its emission profile, and its shift from $7.7 \mu \mathrm{m}$ is not fully understood (e.g., see Peeters et al. 2002; van Diedenhoven et al. 2004; Sloan et al. 2005, 2007). Sloan et al. (2005) suggest that the $7.7 \mu \mathrm{m}$ shift might be influenced by not the charge state but the size of PAHs. In Figure 14, we compare $a_{\mathrm{p}}$ and the peak wavelength of the $7.7 \mu \mathrm{m}$ feature $\left(\lambda_{7.7}\right)$, which shows a trend between $a_{\mathrm{p}}$ and the peak wavelength: as PAH molecules become smaller, $\lambda_{7.7}$ tends to shift toward longer wavelengths. We perform a simple linear-fit in logarithmic scale of $\left\langle a_{\mathrm{p}}\right\rangle$, which gives $\log \left\langle a_{\mathrm{p}}\right\rangle=6.6_{-1.3}^{+1.3}-0.75_{-0.17}^{+0.17} \times\left(\lambda_{7.7} / \mu \mathrm{m}\right)$, $1.2_{-2.0}^{+2.0}-0.07_{-0.25}^{+0.25} \times\left(\lambda_{7.7} / \mu \mathrm{m}\right)$, and $5.1_{-1.1}^{+1.1}-0.56_{-0.14}^{+0.14} \times\left(\lambda_{7.7} / \mu \mathrm{m}\right)$ for the PAH-dominated sources, the silicate-dominated, and combined data sets, respectively.

This trend can be elucidated by postulating that the $\mathrm{C}-\mathrm{C}$ stretching mode acts like a classical harmonic oscillator (see Figure 15). If two carbon atoms oscillate, the wavelength

of the oscillation is given as $\lambda_{\mathrm{C}-\mathrm{C}}=2 \pi c \sqrt{m / k}$, where $c$ is the speed of light, $m$ is the reduced mass ( $m \approx 6 m_{\mathrm{H}}$, where $m_{\mathrm{H}}$ is the mass of the hydrogen nuclei) of the two carbon atoms, and $k$ is the spring constant, which measures the strength of the $\mathrm{C}-\mathrm{C}$ stretch in this case. Considering that the $\mathrm{C}-\mathrm{C}$ stretch of a small $\mathrm{PAH}$ molecule can oscillate more easily than that residing in a large PAH molecule (i.e., smaller $k$ for smaller PAHs than for larger PAHs), $\lambda_{\mathrm{C}-\mathrm{C}}$ would be red-shifted for smaller PAHs with respect to larger PAHs. Although this approach might be oversimplified, however, it gives an idea how the size of PAHs can affect the $7.7 \mu \mathrm{m}$ shift physically. We note that some quantum-chemical computations based on the hybrid density functional theoretical method (B3LYP) have reported that larger PAH molecules tend to shift the peak wavelengths of the 6.2 and $7.7 \mu \mathrm{m}$ features to longer wavelengths (e.g., see Bauschlicher et al. 2010, Ricca et al. 2012). However, it is well known that the vibrational frequencies computed from density functional theory are not accurate and should be scaled in order to agree with the experimentally-measured frequencies. The commonly-adopted frequency scaling factors (e.g., Andersson et al. 2005, Merrick et al. 2007, Borowski 2012) were derived from small molecules ( $\approx 20 \mathrm{C}$ atoms). It is not clear whether

\footnotetext{
${ }^{5}$ The first data release of Gaia is scheduled on 14 September 2016, which will include the positions and $\mathrm{G}$ magnitude for about one billion stars using observations taken between 25 July 2014 and 16 September 2015. See more at http://sci.esa.int/gaia/.
} 


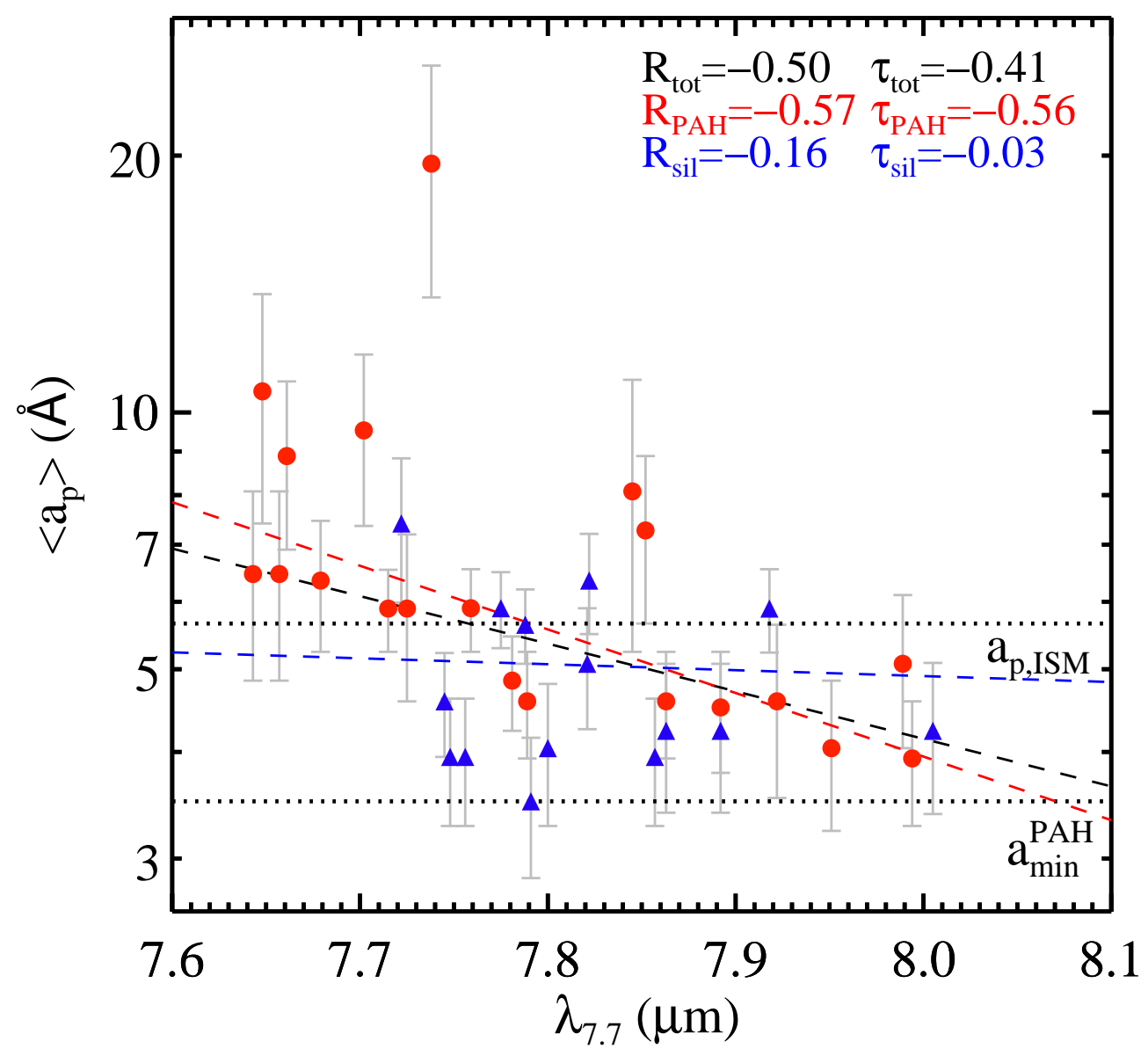

Fig. 14.- Comparison between $a_{\mathrm{p}}$ and the peak wavelength of the $7.7 \mu \mathrm{m}$ PAH feature $\left(\lambda_{7.7}\right)$. 


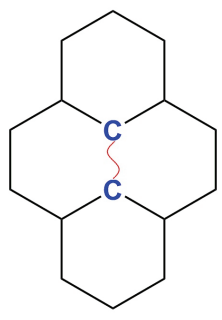

$\mathrm{C}_{16} \mathrm{H}_{10}$

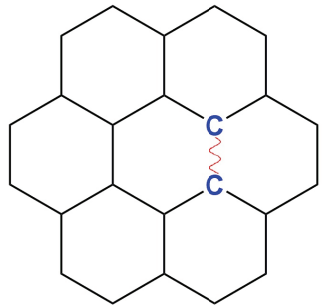

$\mathrm{C}_{24} \mathrm{H}_{12}$

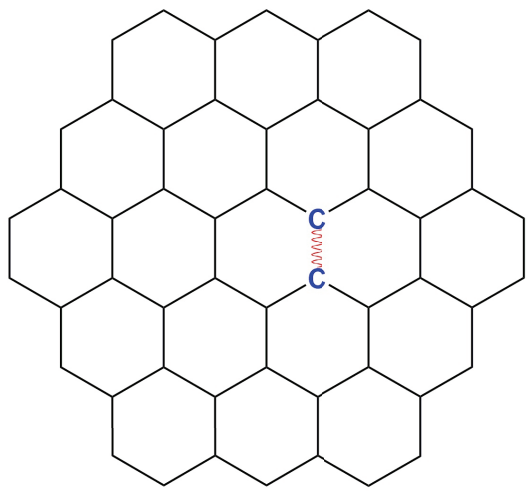

$\mathrm{C}_{54} \mathrm{H}_{18}$

Fig. 15.- Illustration of PAH molecules with three different sizes: pyrene $\left(\mathrm{C}_{16} \mathrm{H}_{10}\right)$, coronene $\left(\mathrm{C}_{24} \mathrm{H}_{12}\right)$, and circumcoronene $\left(\mathrm{C}_{54} \mathrm{H}_{18}\right)$. In each PAH molecule, two carbon atoms connected by a spring delineate the $\mathrm{C}-\mathrm{C}$ stretching mode at $7.7 \mu \mathrm{m}$ resembling a classical harmonic oscillator. Different stiffness of springs represents the variation of the spring constant $(k)$ where the wavelength of the oscillation is defined as $\lambda_{\mathrm{C}-\mathrm{C}}=2 \pi c \sqrt{m / k}(c$ : the speed of light, $\left.m \approx 6 m_{\mathrm{H}}\right)$, see text). The stiffness of $k$ increases from smaller PAHs to larger PAHs.

they are valid for large molecules ( $\gtrsim 100 \mathrm{C}$ atoms) of interest here.

It has been pointed out that the $7.7 \mu \mathrm{m}$ shift is related to the effective temperature of the central star (e.g., see Sloan et al. 2007; Tielens 2008). Sloan et al. (2007) examined the $\mathrm{PAH}$ features seen in a wide range of stellar types including $\mathrm{T}$ Tauri, HAeBe, and post-AGB stars and found that cooler stars show more red-shifted $7.7 \mu \mathrm{m}$ features (see their Figure 5 ). This dependency is consistent with the correlations of $a_{\mathrm{p}}$ with $T_{\text {eff }}$ and $\lambda_{7.7}$ (see Figure 11 and 14). Small PAHs, which tend to emit at a larger $\lambda_{7.7}$, seem to be dominant in disks around cool stars, so the $7.7 \mu \mathrm{m}$ shift could be commonly observed around cool stars.

\section{3. $\quad$ Destruction of PAHs in PPDs}

Photodissociation is one of the primary mechanisms for the destruction of PAHs in PPDs. When PAHs, in particular small PAHs, excited by an energetic photon, cannot redistribute the absorbed energy from the photon via internal vibrational modes, they may lose a hydrogen atom, a hydrogen molecule, or an acetylene molecule $\left(\mathrm{C}_{2} \mathrm{H}_{2}\right)$ and could be eventually photodissociated. We follow the photophysics of Li \& Lunine (2003, see their Appendix A for details) to derive the photodestruction rate $\left(k_{\mathrm{des}}\right)$ approximated by the ejection rate of an acetylene molecule. The destruction timescale $\left(\tau_{\text {des }} \equiv 1 / k_{\text {des }}\right)$ depends on $T_{\text {eff }}, L_{\star}$, and 


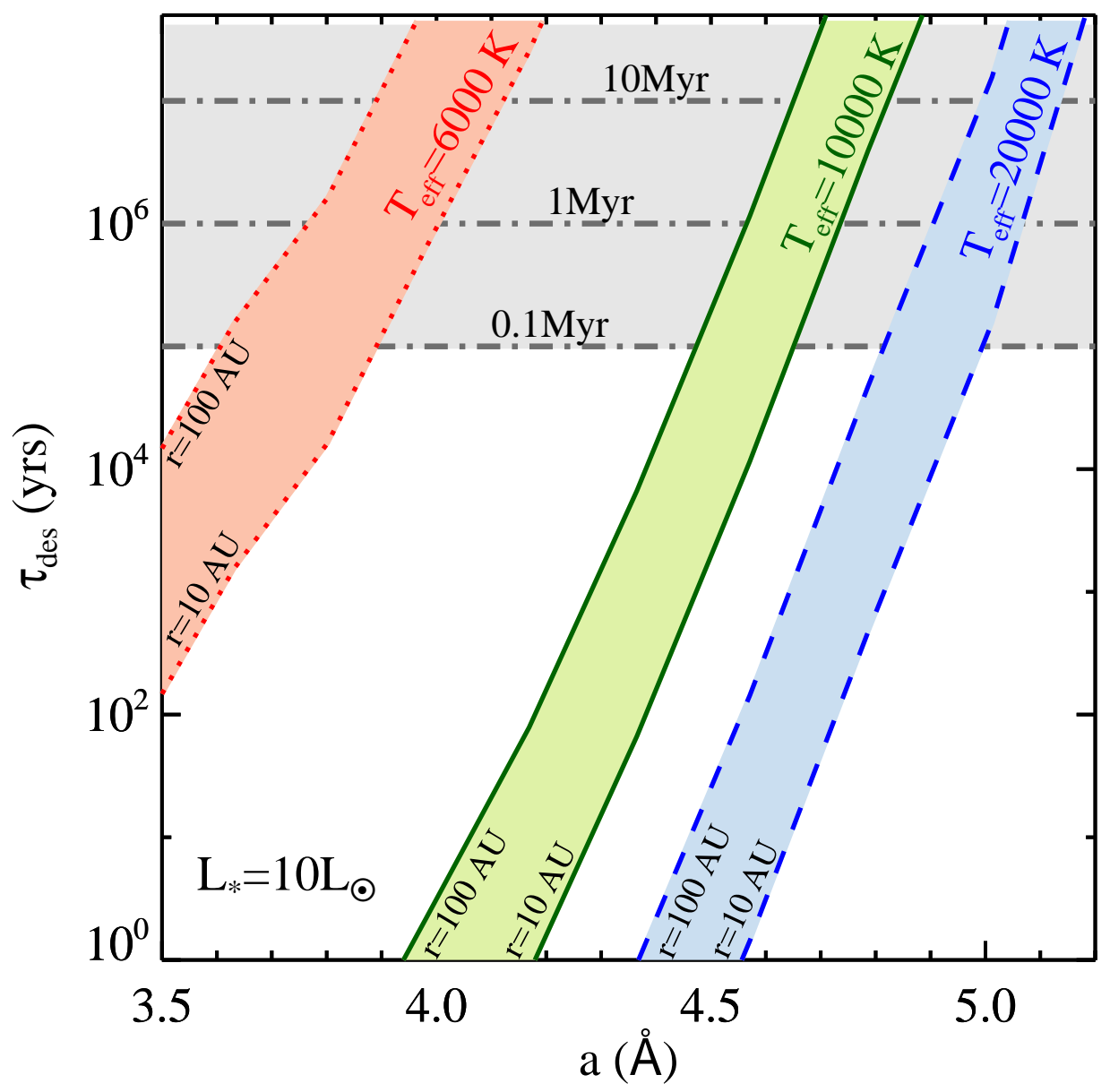

Fig. 16.- Photodestruction timescales $\left(\tau_{\text {des }}\right)$ of PAHs as a function of size for $T_{\text {eff }}=0.6,1$, and $2 \times 10^{4} \mathrm{~K}$ at a given luminosity $\left(L_{\star}=10 L_{\odot}\right)$. For each $T_{\text {eff }}, \tau_{\text {des }}$ at $r=10$ and $100 \mathrm{AU}$ are calculated. A typical range of stellar ages of PPDs (i.e., 0.1-50 Myr) is designated by the shaded region with representative values of $0.1,1$, and $10 \mathrm{Myr}$ (dash-dotted lines). Note that $\tau_{\text {des }}$ is proportional to $r^{2}$ and $L_{\star}^{-1}$ (i.e., $\tau_{\text {des }} \propto r^{2} / L_{\star}$ ). 
the distance of PAHs from the central star $(r)$. For a given $L_{\star}\left(=10 L_{\odot}\right)$, we calculate $\tau_{\text {des }}$ for $T_{\text {eff }}=0.6,1$, and $2 \times 10^{4} \mathrm{~K}$ at a distance of $r=10$ and $100 \mathrm{AU}$ (Figure 16). We note that, when we derive $\tau_{\text {des }}$, we do not consider the competition between photodestruction and photoionization. Intuitively, one would expect a longer $\tau_{\text {des }}$ if such a competition is taken into account because the photoionization process offers an additional channel for losing the absorbed stellar UV photons and will somewhat quench the competing photodestruction process. These UV photons lost through photoionization could otherwise potentially dissociate the molecule by kicking off a $\mathrm{H}$ atom, a $\mathrm{H}_{2}$ molecule, and/or a $\mathrm{C}_{2} \mathrm{H}_{2}$ molecule. Due to lack of experimental data, however, it is difficult to quantitatively investigate the branching ratio between photoionization and photodestruction. Recently, Zhen et al. (2015, 2016) experimentally studied this competition by exposing PAH cations to synchrotron radiation 6 They found that, at photon energies below $\sim 13.6 \mathrm{eV}$, photodestruction dominates over photoionization for small PAH cations. This supports our treatment which ignores the competition between photodestruction and photoionization for PAHs in PPDs. Note that most of these PPDs lack photons of $>10 \mathrm{eV}$.

In spite of the variation of $\tau_{\text {des }}$ with $T_{\text {eff }}$, it is clear that in general small PAHs are readily destroyed during the typical lifetime of PPDs. The lifetime of most of the sources in our sample ranges from 1 to $10 \mathrm{Myr}$, then PAHs smaller than $\approx 4 \AA$ are expected to be destroyed by photodissociation. For hotter sources (i.e., $T_{\text {eff }} \gtrsim 10,000 \mathrm{~K}$ ), even PAHs with $a \approx 4.5 \AA$ are destroyed within $\sim 1 \mathrm{Myr}$. This can naturally explain the correlation between $a_{\mathrm{p}}$ and $T_{\text {eff }}$ shown in Figure 11. Those with high $T_{\text {eff }}$ (i.e., $T_{\text {eff }} \gtrsim 20,000 \mathrm{~K}$ ) are usually more luminous (i.e., $\left.L_{\star} \gtrsim 10^{3} L_{\odot}\right)$ so that PAHs $(\lesssim 5 \AA)$ can be rapidly destroyed. Meanwhile, for those with $T_{\text {eff }} \lesssim 5000 \mathrm{~K}$, small PAHs $(\lesssim 4 \AA)$ can survive against the photodissociation. In this context, the large $a_{\mathrm{p}}$ of DoAr $21\left(\left\langle a_{\mathrm{p}}\right\rangle=10.31 \pm 2.94 \AA\right)$ is surprising because it is a cool star $\left(T_{\text {eff }}=5080 \mathrm{~K}\right)$ with a young age $(\sim 0.4 \mathrm{Myr})$. Although our model parameters are considered to be less reliable due to the uncertain subtraction of the silicate absorption feature (see Figure 19 and more in Section [5.5), our results based on the current data indicate the absence of small PAHs in its disk. If the large $a_{\mathrm{p}}$ of DoAr 21 is real, it could be attributed to its unique physical conditions. DoAr 21 is known to be a strong X-ray emitter $\left(T \sim 10^{8} \mathrm{~K}\right)$, which produces sufficient (far-) UV radiation to excite PAHs located at $\gtrsim 100 \mathrm{AU}$ from the central star (e.g., Jensen et al. 2009) 7 High-resolution MIR imaging

\footnotetext{
${ }^{6}$ Zhen et al. (2015 ) considered coronene $\left(\mathrm{C}_{24} \mathrm{H}_{12}{ }^{+}\right)$, ovalene $\left(\mathrm{C}_{32} \mathrm{H}_{14}{ }^{+}\right)$and hexa-peri-hexabenzocoronene $\left(\mathrm{C}_{42} \mathrm{H}_{18}{ }^{+}\right)$cations irradiated by synchrotron radiation in the range of $8-40 \mathrm{eV}$, whereas Zhen et al. (2016) studied a set of eight PAH cations ranging in sizes from 14 to 24 carbon atoms exposed to vacuum UV photons in the $7-20 \mathrm{eV}$ range.

${ }^{7}$ Jensen et al. (2009) suggest that the origin of the observed emission around DoAr 21 including PAH
} 
obtained with the T-ReCS camera on Gemini South reveals that spatially resolved PAH emission feature at $11.3 \mu \mathrm{m}$ shows little or no emission within $\sim 100$ AU and bright emission extended over $\sim 200 \mathrm{AU}$ (Jensen et al. 2009). This supports the efficient destruction of PAHs near the star through photodissociation, and the large $a_{\mathrm{p}}$ would be a corollary to this.

During the evolution of PPDs, a disk experiences a considerable amount of gas-loss and eventually becomes a gas-poor disk (see also Mann et al. 2006). In such a condition, PAHs can be removed from a PPD by radiative pressure (RP) and Poynting-Robertson (PR) drag in addition to photodissociation. As the RP usually overwhelms the PR drag for small particles (e.g., $\lesssim 10 \mu \mathrm{m}$, see Seok \& Li 2015), the RP can play a main role for the removal of PAHs. Following Equation (19) of Li \& Lunine (2003), we derive the ratio of the radiative force to the gravitational force $\left(\beta_{\mathrm{RP}}\right)$ for different $T_{\text {eff }}\left(=0.6,1\right.$, and $\left.2 \times 10^{4} \mathrm{~K}\right)$. As PAHs fall in the Rayleigh limit (see Li 2009), $\beta_{\mathrm{RP}}$ is not sensitive to their size but is directly proportional to $L_{\star} / M_{\star}$ (see Figure 17). For those with $T_{\text {eff }} \gtrsim 6,000 \mathrm{~K}, \beta_{\mathrm{RP}}$ is expected to be larger than unity, which implies that PAHs would be continuously expelled outward from the disk. The timescale of the RP $\left(\tau_{\mathrm{RP}}\right)$ expelling can be estimated by adopting the local dynamical timescale $\left(\tau_{\text {dyn }}\right.$, see Li \& Lunine 2003; Seok \& Li 2015). We calculate $\tau_{\text {dyn }}=2 \pi / \Omega(r)$, where $\Omega(r) \equiv\left(G M_{\star} / r^{3}\right)^{1 / 2}$ is the Keplerian frequency. Then, $\tau_{\mathrm{RP}}$ is derived from

$$
\tau_{\mathrm{RP}}(r) \approx 31.6 \times\left(M_{\star} / M_{\odot}\right)^{-1 / 2}(r / 10 \mathrm{AU})^{3 / 2} \mathrm{yr} .
$$

In general, $\tau_{\mathrm{RP}}$ is even shorter than $\tau_{\text {des }}$ except for very small PAHs (see Figure 16): for example, HD $34700\left(M_{\star} \approx 1.20 M_{\odot}\right)$ has $\tau_{\mathrm{RP}} \approx 110 \mathrm{yr}$ at $r=25 \mathrm{AU}$, and $\tau_{\text {des }} \approx 240 \mathrm{yr}$ and $\tau_{\text {des }} \approx 1.9 \times 10^{6} \mathrm{yr}$ for PAH size of 3.5 and $4.0 \AA$ at $r=25 \mathrm{AU}$, respectively (see Figure 6 in Seok \& Li 2015). Thus, the RP expulsion would be the primary mechanism for the removal of PAHs from the gas-depleted disk.

For gas-rich disks, on the other hand, the effect of gas drag must be taken into account, which might dominate the motion of PAHs in the disks. For example, the pre-transitional disk around HD 169142 could have a gas-drag timescale of only a few decades (see Seok \& Li 2016), which is much shorter than its RP timescale. Although a full treatment of the gas drag using a two-dimensional disk structure is required to properly estimate the gas-drag timescale in PPDs (e.g., see Chiang \& Youdin 2001 for a review), it is clear that the rapid removal of PAHs would take place in PPDs via photodissociation, RP expulsion, and gas

features, dust continuum, and $\mathrm{H}_{2}$ emission could be a small-scale photodissociation region (PDR) rather than a PPD in the final stage of disk clearing, considering its youth $(\lesssim 1 \mathrm{Myr})$ and the extreme radiation environment (i.e., the brightest X-ray source in the $\rho$ Oph cloud core A with large X-ray flares). Unlike PPDs, a continuous replenishment via collisions of planetesimals or outgassing of cometary bodies would not occur in PDRs, so the lack of small PAHs in DoAr 21 can be plausible. 

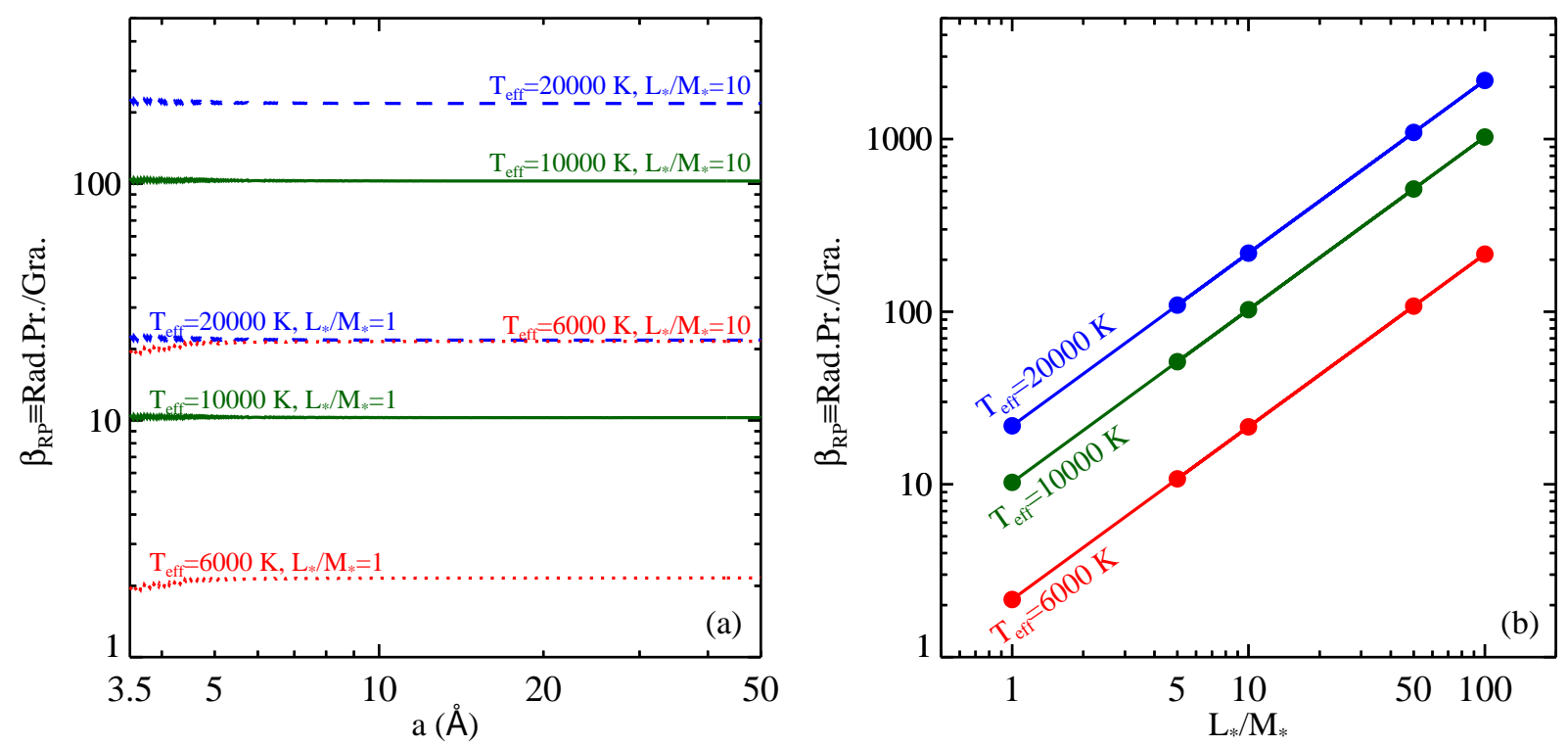

Fig. 17. - Ratio of the radiative force to the gravitational force $\left(\beta_{\mathrm{RP}}\right)$ at various $T_{\mathrm{eff}}(0.6$, 1 , and $\left.2 \times 10^{4} \mathrm{~K}\right) \cdot \beta_{\mathrm{RP}}$ is nearly independent of PAH size (panel (a)), which makes $\beta_{\mathrm{RP}}$ directly proportional to $L_{\star} / M_{\star}$ (panel (b)). $\beta_{\mathrm{RP}}$ is always larger than unity unless both $T_{\text {eff }}$ and $L_{\star} / M_{\star}$ are low. 
drag. To maintain PAHs against the efficient removal of PAHs, continuous replenishment in the disk would be required. A primary mechanism of the replenishment still remains unknown. A continuous supply of PAHs via the outgassing of cometary bodies and collisions of planetesimals and asteroids seems to be the most reasonable way (e.g., see Seok \& Li 2015, 2016). Mann et al. (2007) also discussed the origin and survival of nanoparticles in the inner solar system.

\subsection{Aliphatics versus Aromatics}

While PAH molecules produce conspicuous emission features in the IR spectra of PPDs, relatively weak but distinguishable minor features sometimes appear at $\sim 3.4,6.9$, and $7.3 \mu \mathrm{m}$ (e.g., Sloan et al. 2005; Acke et al. 2010). They are usually attributed to the C-H vibrational modes in aliphatic hydrocarbons (e.g., Chiar et al. 2000; Pendleton \& Allamandola 2002; Yang et al. 2016a, 2016b) although the $3.43 \mu \mathrm{m}$ feature can also originate from anharmonicity and/or superhydrogenation of the aromatic C-H stretch (Barker et al. 1987; Bernstein et al. 1996). Among the $63 \mathrm{MIR}$ spectra that have the spectral data at $\lambda \lesssim 6.9 \mu \mathrm{m}, 21$ sources show the aliphatic feature at $6.9 \mu \mathrm{m}$, which is indicative of aliphatic $\mathrm{CH}$ sidegroups of $\mathrm{PAH}$ molecules in those systems.

For the 21 PPDs, we estimate the aliphatic fraction of the PAH molecules from the measured intensities of the $6.9 \mu \mathrm{m}$ aliphatic feature and $7.7 \mu \mathrm{m}$ aromatic feature $\left(I_{6.9}\right.$ and $I_{7.7}$, respectively) following Li \& Draine (2012). Let $A_{6.9}$ and $A_{7.7}$ be the band strengths of the aliphatic $\mathrm{C}-\mathrm{H}$ deformation band and the aromatic $\mathrm{C}-\mathrm{C}$ stretching band, respectively. We adopt $A_{6.9} \approx 2.3 \times 10^{-18} \mathrm{~cm}$ per $\mathrm{CH}_{2}$ or $\mathrm{CH}_{3}$ group and $A_{7.7}=5.4 \times 10^{-18} \mathrm{~cm}$ per $\mathrm{C}$ atom for charged aromatic molecules (Li \& Draine 2012). Let $B_{\lambda}(T)$ be the Planck function at wavelength $\lambda$ and temperature $T$. If the molecule emits at temperature $T$, the intensity ratio of the $6.9 \mu \mathrm{m}$ aliphatic $\mathrm{C}-\mathrm{H}$ band to the aromatic $\mathrm{C}-\mathrm{C}$ band is approximately $I_{6.9} / I_{7.7} \approx\left[A_{6.9} B_{6.9}(T) N_{\mathrm{C}, \text { aliph }}\right] /\left[A_{7.7} B_{7.7}(T) N_{\mathrm{C}, \text { arom }}\right]$. For a molecule to emit appreciably the PAH bands at $3.3-11.3 \mu \mathrm{m}$, the thermal temperature needs to be in range of $330 \lesssim$ $T \lesssim 1000 \mathrm{~K}$. For such a temperature range we obtain $B_{6.9} / B_{7.7} \approx 0.9 \pm 0.2$ (Li \& Draine 2012). Then, the ratio of the number of $\mathrm{C}$ atoms in aliphatic sidegroups to that in aromatic benzene rings is derived from $N_{\mathrm{C} \text {, aliph }} / N_{\mathrm{C}, \text { arom }} \approx\left(I_{6.9} / I_{7.7}\right) \times\left(A_{7.7} / A_{6.9}\right) \times\left(B_{7.7} / B_{6.9}\right) \approx$ $0.9 \times\left(I_{6.9} / I_{7.7}\right) \times\left(A_{7.7} / A_{6.9}\right)$.

Figure 18 shows the derived aliphatic fractions $\left(N_{\mathrm{C} \text {, aliph }} / N_{\mathrm{C} \text {, arom }}\right)$ in comparison with $T_{\text {eff }}$. It is clearly shown that $N_{\mathrm{C} \text {, aliph }} / N_{\mathrm{C} \text {, arom }}$ decreases as $T_{\text {eff }}$ increases. This correlation has been pointed out previously (e.g., Acke et al. 2010), and it is physically expected since the

aliphatic component is more highly susceptible to local physical conditions than the aromatic 


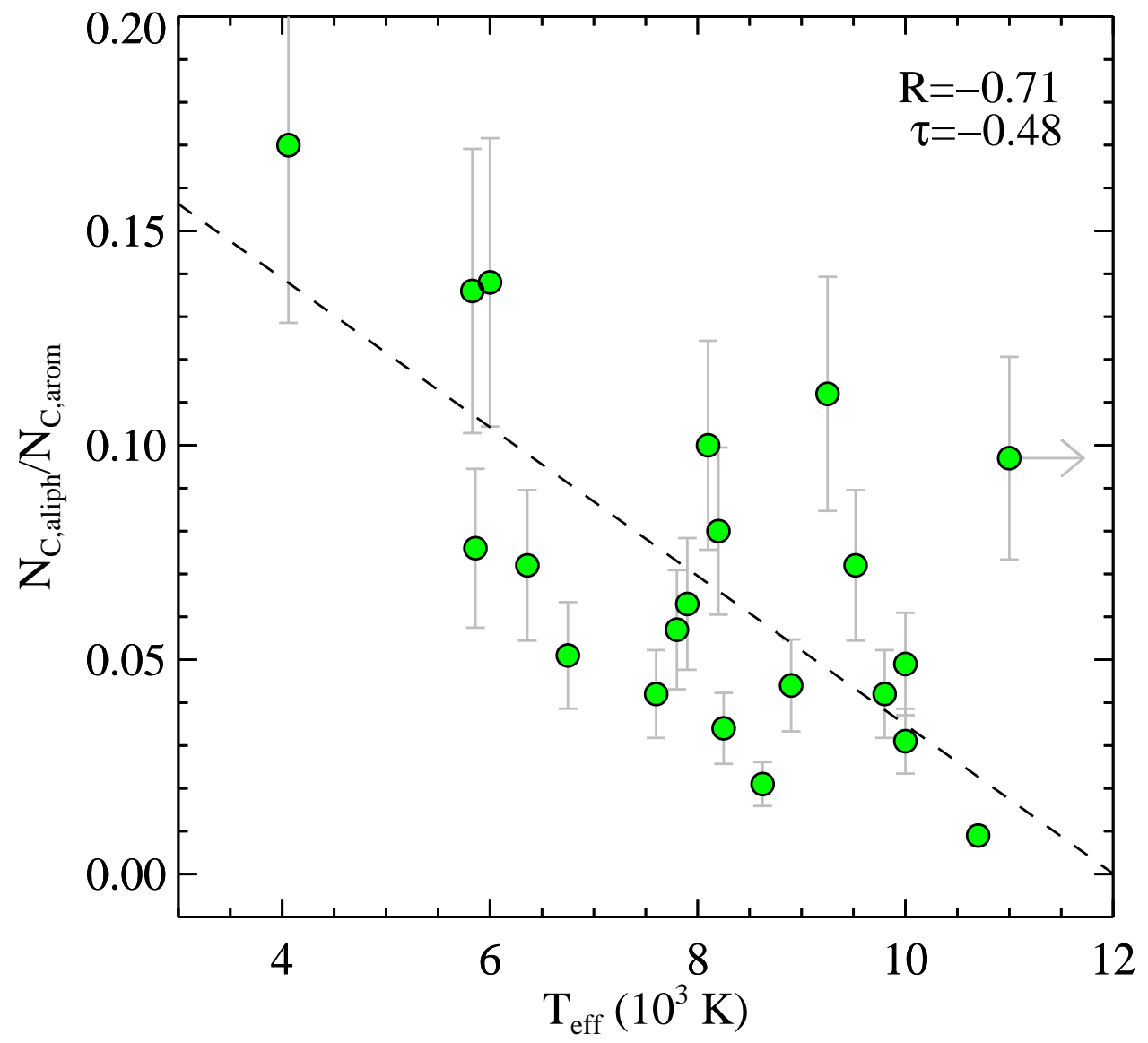

Fig. 18. - Ratios of the number of $\mathrm{C}$ atoms in aliphatic sidegroups to that in aromatic benzene rings $\left(N_{\mathrm{C} \text {, aliph }} / N_{\mathrm{C} \text {, arom }}\right)$ derived from the $6.9 \mu \mathrm{m}$ aliphatic $\mathrm{C}-\mathrm{H}$ deformation band and the $7.7 \mu \mathrm{m}$ aromatic $\mathrm{C}-\mathrm{C}$ stretch band in correlation with $T_{\text {eff }}$. The correlation is linearly fitted $\left(N_{\mathrm{C} \text {, aliph }} / N_{\mathrm{C} \text {, arom }}=0.21 \pm 0.03-0.017 \pm 0.004 \times\left(T_{\text {eff }} / 10^{3} \mathrm{~K}\right)\right.$, dashed line $)$, and the correlation coefficient is measured as $R=-0.71$. For clarity, Wray 15-1484 is plotted with an arrow, which originally falls at $T_{\text {eff }}=30,000 \mathrm{~K}$. Note that we exclude Wray 15-1484 for the correlation analysis (see Section 5.4). 
rings (e.g., Joblin et al. 1996). The aliphatic component would be easily destroyed in harsh environment (e.g., $T_{\text {eff }} \gtrsim 10^{4} \mathrm{~K}$ ), so $N_{\mathrm{C} \text {, aliph }} / N_{\mathrm{C} \text {, arom }}$ shows an anti-correlation with $T_{\text {eff }}$, and even the aliphatic features are not present for those with high $T_{\text {eff }}$.

In this context, the presence of aliphatic features in Wray $15-1484\left(T_{\text {eff }}=30,000 \mathrm{~K}\right)$ remains exceptional $\left(N_{\mathrm{C} \text {, aliph }} / N_{\mathrm{C} \text {, arom }} \approx 0.1\right.$, see Figure 18). The nature of Wray 15-1484 is still controversial. A protoplanetary nebula or a pre-main-sequence star is a most likely scenario (e.g., Lachaume et al. 2007), and the NIR polarization supports an optically thin disk around a HAeBe star (Pereyra et al. 2009). Lachaume et al. (2007) resolved the innermost part of the circumstellar matter at $N$-band $(8-13 \mu \mathrm{m})$ with the Mid-Infrared Interferometric instrument (MIDI) on board the Very Large Telescope Interferometer (VLTI) and pointed out a large gap ( $\sim 30 \mathrm{AU}$ in radius) with a possible presence of an envelope or wind in addition to the disk. If an additional envelope exists, the detected aliphatic features may be ascribed to the envelope where the environment is mild enough for the aliphatic component to survive.

\subsection{Effect of Continuum-Subtraction}

As mentioned in Section 2.3, it is inevitable to force the baselines between the PAH features in the residual spectrum to reach a zero value when we use a spline fit to subtract the continuum from the original spectrum. Hence, there would be a chance to oversubtract the continuum, so we examine the effect of the continuum-subtraction by comparing between a spline fit and PAHFIT for two exemplary sources (DoAr 21 and Oph IRS48). Since PAHFIT can handle major and minor PAH features, various dust features including the silicate absorption bands at 9.7 and $18 \mu \mathrm{m}$ (Smith et al. 2007), we select DoAr 21 and Oph IRS48 as a representative of those with the $9.7 \mu \mathrm{m}$ silicate absorption feature and those with the PAH-dominated spectra, respectively.

The original spectra of the two sources before the continuum-subtraction are shown in Figures 19(a) and (b). Two continua derived from a cubic spline and PAHFIT are overlaid (dotted and dashed lines, respectively), which clearly shows the difference between the two methods. Note that the continuum of PAHFIT is the combination of the total dust continuum and the starlight. Since broad underlying plateaus commonly attributed to large PAH clusters (i.e., 100-1000 carbon atoms; Tielens 2008) are considered as a continuum in a spline fit while they are individually fitted as a PAH feature in PAHFIT, differences at 6-9 $\mu \mathrm{m}$ and 11-14 $\mu \mathrm{m}$ between the two continua, in particular for DoAr 21, are substantial. Even for wavelength ranges without any significant features such as the $9-11 \mu \mathrm{m}$ range, the absolute level of the PAHFIT continuum is lower than that of the spline continuum, so the 


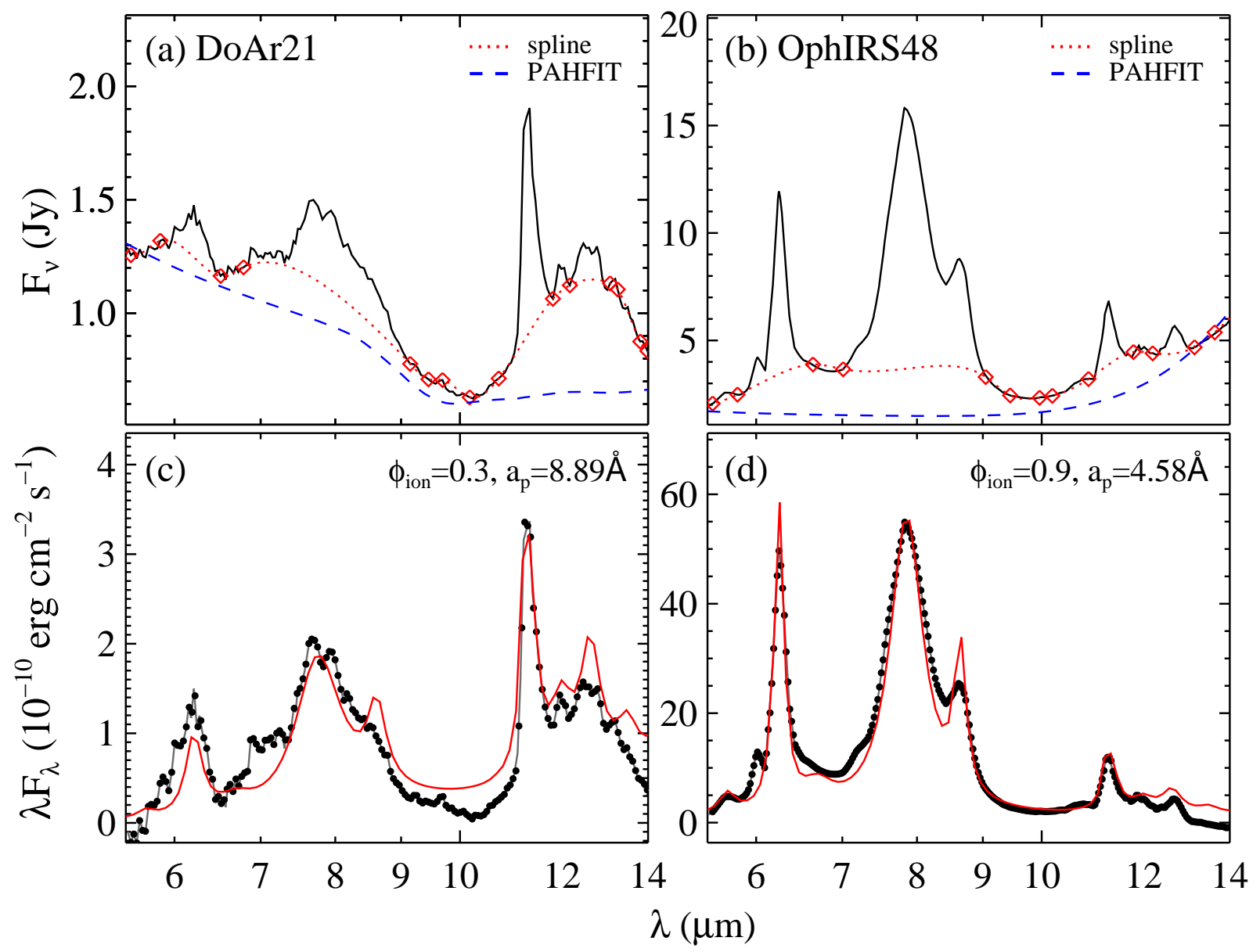

Fig. 19.- (a)-(b) Original spectra (solid lines) of two exemplary sources (DoAr 21 and Oph IRS48) with a comparison between a spline fit (dotted lines) and PAHFIT (dashed lines) for continuum-subtraction. Anchor points used for a spline fit are marked with diamond symbols. In panels (c) and (d), residual spectra of the two sources using PAHFIT are shown with their best-fit model (red lines). Symbol designation is the same as Figure 1. 
baselines of the residual spectra are usually greater than zero (see Figure 19(c) and (d)). In comparison with the residual spectra from the spline fit (DoAr 21 and Oph IRS48 shown in Figures 11 and 7, respectively), the peak intensities of the PAHFIT residual spectra are greater by a factor of $\sim 1.2-1.4$.

We model the residual spectra from PAHFIT, and the best-fit models are shown in Figure 19(c) and (d). For both sources, the best-fit models reproduce the residual spectra more closely than the results using the spline fit (see Figures 1 and 7), and $\chi^{2} /$ dof decreases by a factor of $\sim 100$. However, the best-fit model parameters show the consistent properties of PAHs (i.e., large, neutral PAHs in DoAr 218 and slightly small, ionized PAHs in Oph IRS48), and the median values of the top 70 models for each parameter also agree with each other $\left(\operatorname{DoAr} 21:\left\langle a_{\mathrm{p}}\right\rangle=15.22 \pm 6.52 \AA\right.$ and $\left\langle\phi_{\text {ion }}\right\rangle=0.4 \pm 0.1$ for PAHFIT, $\left\langle a_{\mathrm{p}}\right\rangle=10.31 \pm 2.94 \AA$ and $\left\langle\phi_{\text {ion }}\right\rangle=0.3 \pm 0.1$ for spline, Oph IRS48: $\left\langle a_{\mathrm{p}}\right\rangle=5.64 \pm 1.05 \AA$ and $\left\langle\phi_{\text {ion }}\right\rangle=0.9 \pm 0.1$ for PAHFIT, $\left\langle a_{\mathrm{p}}\right\rangle=7.27 \pm 1.62 \AA$ and $\left\langle\phi_{\text {ion }}\right\rangle=0.7 \pm 0.1$ for spline). This indicates that the correlations between the PAH parameters and the stellar properties that we found (Figures 11-14) would not be altered significantly by the exact choice of continuum subtraction. In addition, we check the effect on the total mass of PAHs, which is directly affected by the absolute scale of the residual spectra. We found $M_{\mathrm{PAH}}^{10 \mathrm{AU}}=2.91$ and $2.62 \times 10^{-5} M_{\oplus}$ for DoAr 21 and Oph IRS48, respectively, which increase by a factor of $\sim 1.1-2.4$. This implies that $M_{\mathrm{PAH}}$ derived in Section 4 could be underestimated slightly (i.e., $\sim 10-20 \%$ ) for those with prominent PAH features and by a factor of $\sim 2$ for those with weak PAH features.

The total mass of PAHs at $10 \mathrm{AU}\left(M_{\mathrm{PAH}}^{10 \mathrm{AU}}\right)$ listed in Table 2 and its possible underestimation may give one an impression that those sources with prominent PAH features tend to have a lower mass than those with weak PAH features. This is most likely due to the different starlight intensities for different sources: those with strong $\mathrm{PAH}$ features are usually HAeBe stars with relatively high $T_{\text {eff }}$ (i.e., high $U$, e.g., HD 85667: $T_{\text {eff }}=20900 \mathrm{~K}$, MWC 297: $T_{\text {eff }}=24000 \mathrm{~K}$ ), whereas those with weak PAH features are mostly TTSs with low $T_{\text {eff }}$ (i.e., low $U$, e.g., EC82: $T_{\text {eff }}=4060 \mathrm{~K}$, IC348LRL110: $T_{\text {eff }}=3778 \mathrm{~K}$ ). Even if the PAH features from a source with low $U$ are weak, one may still require a large amount of PAHs to account for the observed PAH emission because of its low $U\left(M_{\mathrm{PAH}} \propto 1 / U\right)$. Moreover, the detected PAH features from these low- $U$ sources might be biased toward unusual cases emitting stronger PAH emission relative to other sources with similar $U$ (possibly due to

\footnotetext{
${ }^{8}$ Although we categorize the model parameters of DoAr 21 as less reliable due to its relatively weak PAH features and the rough subtraction of the $9.7 \mu \mathrm{m}$ silicate absorption feature (Table 2), it seems that the PAHFIT results consistently indicate the existence of large PAHs (i.e., the lack of small PAHs) in this disk. This supports that the unique environment of DoAr 21 results in the efficient destruction of PAHs (see Section 5.3.
} 
more abundant PAHs) since we might not be able to detect those whose PAH feaures are weaker. Lastly, $M_{\mathrm{PAH}}^{10 \mathrm{AU}}$ does not represent the actual total mass of PAHs in PPDs. It must be reviewed taking into account the spatial distribution of PAHs in each disk, which will give a true insight into the relation between the stellar properties and the PAH mass in PPDs.

\section{Summary}

We have accumulated a sample of 69 PPDs reported to show PAH features in the literature and have presented their IR spectra collated from the literature and data archives. Our sample includes 14 TTSs and 55 HAeBe stars, which allows us to examine PAHs in PPDs with a wide range of stellar parameters. We have modeled the PAH emission of 61 PPDs, excluding eight PPDs of which the IR spectra are not adequate for model calculations, and the best-fit models mostly provide an excellent fit to the PAH emission features of the PPDs. In the model calculations, the size distribution of PAHs characterized by the peak and the width of the log-normal distribution $\left(a_{0}\right.$ and $\left.\sigma\right)$ as well as the ionization fraction $\left(\phi_{\text {ion }}\right)$ are derived coherently for the large sample of PPDs. Our principle results are as follows:

1. For the 61 PPDs, the peak of the PAH mass distribution $\left(a_{\mathrm{p}}\right)$ is calculated from $a_{0}$ and $\sigma$, which represents the overall PAH size distributions. It is found that $a_{\mathrm{p}}$ shows a positive correlation with the stellar effective temperature $\left(T_{\text {eff }}\right)$, indicating that small PAHs are dominant in disks around cool stars while large PAHs are in those associated with hot stars. This trend is a natural result from the photodissociation of PAHs induced by energetic photons, which destroy small PAHs more rapidly than large PAHs. The peak size $a_{\mathrm{p}}$ also shows a correlation with $L_{\star} / M_{\star}$, which appears as tight as with $T_{\text {eff }}$. This is probably due to the dependence of $L_{\star}$ on $T_{\text {eff }}\left(L_{\star} \propto T_{\text {eff }}^{4}\right)$.

2. The peak size $a_{\mathrm{p}}$ does not exhibit any tight correlation with the stellar age, but the overall trend indicates that $a_{\mathrm{p}}$ decreases with the stellar age. To maintain the abundance of small PAHs against complete destruction by photodissociation, small PAHs are required to be replenished rapidly. This might imply that a continuous supply of PAHs via the outgassing and collisions of planetesimals, asteroids, and cometary bodies is favored as a primary mechanism of the replenishment.

3. Unlike $a_{\mathrm{p}}, \phi_{\text {ion }}$ shows no significant correlation with $T_{\text {eff }}$. This lack of correlation is attributed to the diversity of the spatial distribution of PAHs in disks from one to another. Since $\phi_{\text {ion }}$ depends on $U(r)\left(U \propto r^{-2}\right)$, the distance of PAHs from the central star should be taken into account to properly interpret $\phi_{\text {ion }}$.

4. The shift of the peak wavelength of the $7.7 \mu \mathrm{m}$ PAH feature $\left(\lambda_{7.7}\right)$ shows a moderate 
correlation with $a_{\mathrm{p}}$ : as PAH molecules become smaller, $\lambda_{7.7}$ tends to shift toward longer wavelengths. This is consistent with the negative correlation between $\lambda_{7.7}$ and $T_{\text {eff }}$ that has been previously reported because small PAHs are dominant in disks around cool stars (i.e., a positive correlation between $a_{\mathrm{p}}$ and $\left.T_{\text {eff }}\right)$.

5. Twenty one sources in the sample show the aliphatic feature at $6.9 \mu \mathrm{m}$, indicating aliphatic $\mathrm{CH}$ sidegroups of PAHs in those systems. The aliphatic fractions $\left(N_{\mathrm{C} \text {, aliph }} / N_{\mathrm{C} \text {, arom }}\right)$ tend to decrease with increasing $T_{\text {eff }}$ since the aliphatic component is more susceptible to local physical conditions such as $T_{\text {eff }}$ than the aromatic rings.

Increasing the number of PPDs with spatially resolved PAH emission obtained with ground-based telescopes as well as unprecedented results from the up-coming space mission, James Webb Space Telescope, will enable us to more precisely quantify the physical properties of PAHs, in particular the total mass of PAHs, and to better answer the role of PAHs in the evolution of PPDs and possible planet formation.

We thank X. J. Yang for her kind assistance with Figure 15 and V. C. Geers for generously providing the VLT data. We thank X. J. Yang, H. Zhang, and the anonymous referee for very helpful discussions and suggestion. We are supported in part by NSF AST-1109039, NNX13AE63G, NSFC 11173019, and the University of Missouri Research Board. 


\section{A. Brief description on the individual sources}

\section{A.1. AB Aur}

$\mathrm{AB}$ Aur is one of the well-known transitional disks, exhibiting a truncated dusty disk at an inner radius of $\sim 70 \mathrm{AU}$ (Piétu et al. 2005) with a compact inner disk (Tang et al. 2012). NIR observations show the presence of spiral arms in the disk (Fukagawa et al. 2004). The dust disk is highly asymmetric and shows a horseshoe morphology, which is interpreted as the birthplace of future planets. Hashimoto et al. (2011) have estimated an upper limit of 5 $M_{J}$ for a possible companion of AB Aur although planets have not yet been detected in the disk.

\section{A.2. AK Sco}

AK Sco A/B (a.k.a. HD 152404A/B) is a binary system composed of two nearlyidentical F5 stars in an eccentric orbit. The orbital inclination is constrained to the range of $65^{\circ}<i<70^{\circ}$ (Alencar et al. 2003), but eclipses have not been detected. Although the IR spectra do not show any significant MIR-variability (Kóspál et al. 2012), the optical spectra of AK Sco exhibit emission or absorption features that show variation in shape (Alencar et al. 2003). This indicates the presence of outflow and infall activites in this system. Recent observations with Hubble Space Telescope (HST) reveal a drop of the $\mathrm{H}_{2}$ flux by up to $\sim 10 \%$ caused by gas infalling (Gómez de Castro et al. 2016).

\section{A.3. $\mathrm{BD}+40^{\circ} 4124$}

$\mathrm{BD}+40^{\circ} 4124$ (a.k.a. V1685 Cyg, MWC 340) is one of the youngest objects in our sample (e.g., Manoj et al. 2006; Liu et al. 2011). BD+40²124 is located within a small pre-mainsequence star cluster (e.g., Sandell et al. 2011), consisting of two HAeBe stars (BD+40 4124 and $\mathrm{LkH} \alpha 224$ ) and an embedded binary system, $\mathrm{LkH} \alpha 225$ (=V1318 Cyg). Sub-millimeter observations reveal that $\mathrm{BD}+40^{\circ} 4124$ is not associated with any significant disk continuum emission, instead, the extended emission from the mother cloud dominates the observed submm emission (Sandell et al. 2011). The Spitzer $70 \mu \mathrm{m}$ image shows extended emission features near $\mathrm{BD}+40^{\circ} 4124$, which likely originate from heated dust from the reflection nebulosity rather than the PPD. In this context, its MIR spectrum is dominated by PAH emission features, of which the profiles are similar to those of diffuse ISM, and this indicates the origin of the PAH emission from the reflection nebulosity. 


\section{A.4. BF Ori}

The Herbig Ae star BF Ori is known to have strong photometric variability (Shevchenko et al. 1993), which is similar to that of UX Ori. MIR spectra also show variability of the $9.7 \mu \mathrm{m}$ silicate emission feature over a decade (Kóspál et al. 2012). An edge-on circumstellar disk is indicated from an anti-correlated linear polarization (Grinin et al. 1991). Episodic accretion around BF Ori has been monitored (de Winter et al. 1999), suggesting evaporation of star-grazing bodies, which is also called the " $\beta$ Pic phenomenon" (e.g., Grinin et al. 1996).

\section{A.5. DoAr 21}

DoAr 21 (a.k.a. Haro 1-6, ROXR1 13, RXJ1626.03-2423, V2246 Oph) is one of the brightest X-ray weak-lined TTS (WTTS; Neuhäuser et al. 1994). An extended ring structure is detected at NIR $\mathrm{H}_{2}$ emission (Panic 2009, Ph.D. Thesis), which is at $\sim 73-219$ AU away from the central star. This is consistent with the estimation of a cavity size of $r_{\text {cav }}=70_{-10}^{+30} \mathrm{AU}$ (van der Marel et al. 2016). Jensen et al. (2009) claimed that the observed $\mathrm{PAH}, \mathrm{H}_{2}$, and continuum emission can be associated with a small scale PDR rather than a PPD.

\section{A.6. $\quad$ EC 82}

EC 82 with a spectral type of K7-K8 (Sturm et al. 2013; Rigliaco et al. 2015) is associated with the Serpens cluster. Its VLT/VISIR $N$-band spectrum is dominated by the strong $9.7 \mu \mathrm{m}$ silicate emission feature (Geers et al. 2007b), and the tentative detection of the $11.2 \mu \mathrm{m}$ PAH feature in their spectrum gives a radial spatial extent of $\lesssim 0^{\prime \prime} .23$ (i.e., $\lesssim 95 \mathrm{AU}$ at $415 \mathrm{pc})$. This object is one of the coolest stars $\left(T_{\text {eff }} \approx 4060 \mathrm{~K}\right)$ that exhibit PAH features in their IR spectra. This provides observational support to the theory of Li \& Draine (2002) which suggests that the excitation of PAHs does not require UV photons, instead, visible and NIR photons are capable of exciting PAHs sufficiently to emit at the PAH bands (also see Mattioda et al. 2005).

\section{A.7. HD 31648}

The isolated Herbig Ae star HD 31648 (a.k.a. MWC 480) has a spectral type of A2pshe (Keller et al. 2008), located in the Taurus-Auriga complex. A large circumstellar disk around HD 31648 has been spatially resolved in CO and (sub-)mm continuum (e.g., Simon et al. 
2000; Hamidouche et al. 2006; Piétu et al. 2007; Sandell et al. 2011), but the disk is not seen in coronagraphic NIR images obtained with HST (Augereau et al. 2001). While ${ }^{12} \mathrm{CO}$ $J=2-1$ and sub-mm (480 and $850 \mu \mathrm{m}$ ) emissions are extended up to $\sim 8^{\prime \prime}$ (e.g., Piétu et al. 2007; Sandell et al. 2011), the $1.4 \mathrm{~mm}$ continuum shows a disk size of only $0.8 \times 0.7$ (Hamidouche et al. 2006).

\section{A.8. HD 34282}

HD 34282 is a Herbig Ae star (A0V-A3V, e.g., Merín et al. 2004; Keller et al. 2008), and its distance is rather uncertain. The Hipparcos parallax gives a distance of $164_{-30}^{+60} \mathrm{pc}$, which results in an "anomalous position" of HD 34282 in the H-R diagram due to a low luminosity (i.e., well below the expected luminosity of a star of its spectral type, $L_{\star}=4.8 L_{\odot}$; van den Ancker et al. 1998). Based on the Keplerian motion inferred from CO observations (Piétu et al. 2003), its distance is newly constrained as $d=400_{-100}^{+170} \mathrm{pc}$, which yields $L_{\star}=29_{-13}^{+30} L_{\odot}$ more consistent with the stellar properties expected for the spectral type of HD 34282 (Merín et al. 2004). Its disk has been spatially resolved at $1.3 \mathrm{~mm}$ (Piétu et al. 2003) but is unresolved at 480 and $850 \mu \mathrm{m}$ with SCUBA on the James Clerk Maxwell Telescope (JCMT; Sandell et al. 2011). A spatially resolved MIR $Q$-band image obtained with Gemini north/MICHELLE infers the presence of a large dust gap in the disk $\left(r_{\text {cav }}=92_{-17}^{+31} \mathrm{AU}\right.$; Khalafinejad et al. 2016).

\section{A.9. HD 34700}

HD 34700 (a.k.a. SAO 112630) has a spectral type of G0IVe (Schütz et al. 2009) and is probably in transition from a TTS to a Vega-like star (e.g., Seok \& Li 2015). Its distance is very uncertain, ranging from $\sim 100$ to $430 \mathrm{AU}$ (e.g., Acke \& van den Ancker 2004; Torres 2004; Seok \& Li 2015), and we adopt 260 AU following Seok \& Li (2015). The HD 34700 system composed of a spectroscopic binary (Torres 2004) and two other faint components is confirmed with $J H K$-band images and optical spectroscopy (Sterzik et al. 2005). Although it has not been spatially resolved, analysis of its SED indicates the dust spatial distribution of the HD 34700 disk peaks at $\approx 100 \mathrm{AU}$ and decreases outward with a power-law index of $\approx 2$ (Seok \& Li 2015). The PAH parameters derived in this work $\left(\left\langle a_{0}\right\rangle=2.5 \pm 0.5 \AA\right.$, $\langle\sigma\rangle=0.3 \pm 0.1$, and $\left.\left\langle\phi_{\text {ion }}\right\rangle=0.2 \pm 0.1\right)$ are consistent with the previous results from Seok $\& \mathrm{Li}$ (2015), which indicate small PAH population $\left(a_{0}=3.5 \AA\right.$ and $\left.\sigma=0.3\right)$ with a mixture of neutral and ionized PAHs: for small PAHs $(a \lesssim 5 \AA), \phi_{\text {ion }} \sim 0.2$ at $r=25 \mathrm{AU}$ (see their Figure 3). 


\section{A.10. HD 35187}

The Herbig Ae star HD 35187 (a.k.a. BD+24826, SAO 77144) is a young binary system (HD 35187A: A7V, HD 35187B: A2V; Dunkin \& Crawford 1998). The separation of two stars is by $\sim 1$ '.39 at a distance of 150 pc. Dunkin \& Crawford (1998) suggested that HD 35187B is surrounded by a substantial disk of gas and dust whereas HD 35187A is not. Therefore, the observed IR emission including PAH features is likely to originate from HD 35187B. HD 35187 is a relatively strong radio source (e.g., Natta et al. 2004), which is dominated by free-free emission and by dust emission at wavelengths shorter than $1.3 \mathrm{~mm}$. SCUBA submm observations did not resolve the source (e.g., Sheret et al. 2004; Sandell et al. 2011). Both

stars are located close to the zero-age main sequence (ZAMS) in the H-R diagram, and the SED of HD 35187 is similar to those of evolved HAeBe stars seen by Waelkens et al. (1994). This implies that this system is in transition between the HAeBe stars and the Vega-like stars (e.g., Dunkin \& Crawford 1998).

\section{A.11. HD 36112}

HD 36112 (a.k.a. MWC 758) has a transition disk around the Herbig Ae star with a spectral type of A5IVe (e.g., van Boekel et al. 2005), which has been extensively observed in multi-wavelengths (e.g., Chapillon et al. 2008; Isella et al. 2008, 2010; Grady et al. 2013; Benisty et al. 2015; Marino et al. 2015). Its disk structure shows spiral arms, non-axisymmetric dust continuum, and axisymmetric $\mathrm{CO}$ emission, which can be associated with a massive planet at $\sim 160 \mathrm{AU}$ (Dong et al. 2015). Dust observations at mm regime infer an inner cavity with a radius of $\sim 73$ AU possibly due to a low-mass companion within $\sim 42 \mathrm{AU}$ (Isella et al. 2010; Andrews et al. 2011). NIR observations with the VLTI (Isella et al. 2008) show strong emission coming from sub-AU scales, indicating that the disk can be classified as a pre-transitional disk (e.g., Espaillat et al. 2007).

\section{A.12. HD 36917}

The Herbig Ae star HD 36917 (a.k.a V372 Ori) is a spectroscopic binary (B9.5+A0.5; Levato \& Abt 1976). HD 36917 is a member of the young Orion Nebula Cluster, which implies that its age is younger than 1 Myr (Manoj et al. 2002). High-resolution MIR imaging at 12 and $18 \mu \mathrm{m}$ with MICHELLE on the Gemini North telescope did not resolve its circumstellar disk (Marinãs et al. 2011), which gives a upper limit of $\lesssim 0$ '.25 for its angular size (i.e., $\lesssim 101 \mathrm{AU}$ at the distance of $\approx 375 \mathrm{pc}$ ). 


\section{A.13. HD 37357}

HD 37357 has a spectral type of A0V and is a member of the Orion OB1c association (Juhász et al. 2010). Its pulsational variability is discovered from high-precision time-series photometry obtained with the Microvariability and Oscillations of Stars (MOST) and Convection, Rotation et Transits Planétaires (CoRoT) satellites (Zwintz et al. 2014).

\section{A.14. HD 37411}

The Herbig Ae star HD 37411 was discovered by Hu et al. (1989) and is classified as a $\lambda$ Boo star (Gray \& Corbally 1998), which is metal deficient but has nearly solar abundances of CNO and S (Morgan et al. 1943). The existence of a disk with an inclination of $63^{\circ}$ is inferred from the SED analysis (Liu et al. 2011). Boersma et al. (2008) reported a strong $11.1 \mu \mathrm{m}$ feature in HD 37411, which is often detected as a minor feature accompanying the $11.2 \mu \mathrm{m}$ PAH feature (Hony et al. 2001). However, the $11.1 \mu \mathrm{m}$ feature seen in HD 37411 is unusually strong relative to the $11.2 \mu \mathrm{m}$ feature. They claimed the presence of warm crystalline silicate in the source based on the presence of the broad $10 \mu \mathrm{m}$ feature, which implies that that the emission from forsterite might account for the $11.1 \mu \mathrm{m}$ feature.

\section{A.15. HD 37806}

HD 37806 (a.k.a. MWC 120) has a spectral type of B9-A2 (Kessler-Silacci et al. 2006) and is a probable variable (van den Ancker et al. 1998). Its circumstellar disk has not been spatially resolved with MICHELLE on the Gemini North telescope (e.g., Marinãs et al. 2011), which gives an upper limit of $0^{\prime \prime} .31$ and $0^{\prime \prime} .33$ at 12 and $18 \mu \mathrm{m}$, respectively.

\section{A.16. HD 38120}

HD 38120 has a spectral type of B9 (e.g., Juhász et al. 2010), and its age estimate ranges from $\lesssim 1.0$ Myr (e.g., Sartori et al. 2010; Manoj et al. 2010) to $\sim 5_{-0.5}^{+0.5}$ Myr (e.g., Alecian et al. 2013) probably owing to different distance assumption (e.g., $d=422 \pm 230$ pc; Donehew \& Brittain 2011). CO emission from its disk has been detected (Dent et al. 2005), and the best-fit to the $J=3-2$ CO line profiles with a simple disk model constrains the properties of the disk, such as dust mass of $\sim 10^{-5} M_{\odot}$ and an outer radius of $\sim 300 \mathrm{AU}$ at the distance of 420 pc. 


\section{A.17. HD 58647}

HD 58647 is a young Herbig Be star (B9IV: e.g., Juhász et al. 2010), of which the age is $\approx 1$ Myr (Mariñas et al. 2011) or even younger (0.4 Myr: van den Ancker et al. 1998, 0.16 Myr: Montesinos et al. 2009). It is located at 543 pc away (Mariñas et al. 2011), but other studies estimate that it can be closer $\left(280_{-50}^{+80} \mathrm{pc}\right.$ : van den Ancker et al. 1998, $318_{-46}^{+65}$ pc: van Leeuwen 2007). Using the $K$-band observations of the Keck interferometer, Monnier et al. (2005) measured the radius of a geometric ring of the $K$-band continuum emission, which corresponds $0.82 \pm 0.13 \mathrm{AU}$ at a distance of $280 \mathrm{pc}$. The $K$-band emission region is considered to arise near the dust sublimation radius of the accretion disk. Some of its hydrogen emission lines (e.g., $\mathrm{H} \alpha, \mathrm{Br} \gamma$ ) appear to have a double-peak profile. NIR observations with high angular and high spectral resolution (e.g., Kurosawa et al. 2016) indicate that the $\operatorname{Br} \gamma$ emission is from a disk wind with a radius of $\sim 0.5 \mathrm{AU}$, which is within the inner radius of the $K$-band continuum-emitting ring (0.68 AU).

\section{A.18. HD 72106}

HD 72106 is a binary system, consisting of the primary star, HD 72106A, identified as a magnetic star (Wade et al. 2005) and the secondary star, HD 72106B, identified as a HAeBe star (Vieira et al. 2003). The binary stars are separated by 0'.805 (ESA 1997) or $\approx 280$ AU at

a distance of $289_{-85}^{+204}$ pc (Alecian et al. 2013). HD 72106B is the one emitting PAH emission as well as other dust emission (e.g., Schütz et al. 2005). Schütz et al. (2005) obtained $N$ band spectra $(8-13 \mu \mathrm{m})$ with the ESO TIMMI2 camera at La Silla observatory. It is found that the spectrum of HD 72106 is dominated by crystalline forsterite and enstatite rather than small, amorphous silicate grains. Also, large amorphous silicates and $\mathrm{SiO}_{2}$ are present. Such dust species are known to result from dust processing in circumstellar disks, and only a few pre-main-sequence stars (e.g., HD 100546, HD 179218) have shown them so far. Its IR spectrum shows a remarkable resemblance with a combination of spectra of the comets Halley and Hale-Bopp, which makes this object interesting for searching for on-going planet formation in its disk. While HD 72106A shows strong peculiarities in chemical abundances (Folsom et al. 2008), the large majority of the chemical elements in HD 72106B is consistent with solar abundances as commonly found in other HAeBe stars (e.g., Acke \& Waelkens 2004). Schegerer et al. (2009) performed interferometric observations in $N$-band with the MIDI at the VLTI and found that a purely passive disk (i.e., no accretion) with an inner and outer disk radii of $\sim 0.5$ and $\sim 40$ AU, respectively. 


\section{A.19. HD 85567}

HD 85567 (a.k.a. V596 Car, Hen 3-331) is a Herbig Be star (B7-8Ve: Juhász et al. 2010) located at $1.5 \pm 0.5 \mathrm{kpc}$ (Verhoeff et al. 2012). The existence of a close binary companion interacting with the circumstellar disk of HD 85567 was proposed (Miroshnichenko et al. 2001 ), and the binary companion with a separation of $\gtrsim 0.5$ is confirmed later (Baines et al. 2006). Vural et al. (2014) performed NIR interferometric observations to investigate the sub-AU scale disk structure of HD 85567 with the AMBER instrument at the VLTI and found that the inner radius of the disk $\left(R_{\text {in }} \sim 0.67 \mathrm{AU}\right.$ with a temperature of $\left.T_{\text {in }} \sim 2200 \mathrm{~K}\right)$ is smaller than that expected from the size-luminosity relation. This implies that an optically thick gaseous inner disk exists to shield the stellar radiation of the central star so that dust can survive closer to the star than the radius of dust sublimation $(T \sim 1500 \mathrm{~K})$.

\section{A.20. HD 95881}

HD 95881, a Herbig Ae star (A2III/IVe: Acke \& van den Ancker 2004), is classified as a group II source following the classification of Meeus et al. (2001). Group II sources have blue SEDs in the MIR spectral range $(10-60 \mu \mathrm{m})$, implying a flat or self-shadowed disk geometry. Although group II sources usually show weak or no PAH emission (e.g., Acke et al. 2010), HD 95881 shows prominent PAH emission as well as [O I] $6300 \AA$, both of which indicate the presence of gas in the upper layers of the disk and direct irradiation by stellar photons. Verhoeff et al. (2010) carried out a comprehensive study toward the disk of HD 95881 using NIR ( $K$-band) and MIR $(10 \mu \mathrm{m})$ interferometric observations with the AMBER and MIDI instruments at the VLTI and $Q$-band imaging and $N$-band spectroscopy with VLT/VISIR. They found that the PAH emission is more extended $(R \sim 100 \mathrm{AU})$ than the dust continuum (mostly located within $\lesssim 2.5 \mathrm{AU}$ ) and concluded that the inner disk has a puffed up inner rim containing most of the grains emitting in the IR wavebands (large grains probably existing deep inside the disk and detectable only in $\mathrm{mm}$ are excluded). Whereas a mixture of gas and PAHs with a flaring disk geometry exists in the outer disk, dust grains are depleted due to coagulation and settled down to the mid-plane.

\section{A.21. HD 97048}

HD 97048 (a.k.a. CU Cha), a Herbig Ae star (A0pshe: Keller et al. 2008), is located at $\sim 150$ pc (Alonso-Albi et al. 2009) or $175_{-20}^{+26}$ pc (van Boekel et al. 2005). This is one of the several PPDs showing prominent nanodiamond features at 3.43 and $3.53 \mu \mathrm{m}$ together with 
the $3.3 \mu \mathrm{m}$ PAH feature in its NIR spectrum (e.g., Habart et al. 2004c). Both PAH and nanodiamond features have been spatially resolved (e.g., Habart et al. 2004c, van Boekel et al. 2004, Habart et al. 2006, Quanz et al. 2012): while the PAH features at 8.6, 11.3 and $12.7 \mu \mathrm{m}$ are extended on a scale of a few $100 \mathrm{AU}$ (van Boekel et al. 2004, 80-370 AU: Quanz et al. 2012), the $3.3 \mu \mathrm{m}$ PAH emission mainly comes from a more confined region (Habart et al. 2006). The diamond features are slightly less extended than the $3.3 \mu \mathrm{m}$ feature (FWHM 41 AU: Habart et al. 2006, $\lesssim 15$ AU: Quanz et al. 2012), and the adjacent continuum is even less extended (FWHM $\sim 23 \mathrm{AU}$ : van Boekel et al. 2004, or $\sim 20-160$ AU for the 3.5-18 $\mu \mathrm{m}$ NIR/MIR continuum: Quanz et al. 2012). Recent observations including ALMA, ATCA, and VLT/SPHERE (Spectro-Polarimetric High-contrast Exoplanet REsearch) have revealed that the HD 97048 disk has several ring (and gap) structures (e.g., Maaskant et al. 2013; Ginski et al. 2016; van der Plas et al. 2016; Walsh et al. 2016), which can be explained by on-going low-mass planet formation (e.g., $\approx 0.7 M_{J}$ at $2.5-11 \mathrm{AU}$ : van der Plas et al. 2016).

Using the CRIRES instrument at the VLT, Carmona et al. (2011) detected $\mathrm{H}_{2}$ 1-0 S(1) line emission at $2.12 \mu \mathrm{m}$ and found that the emission is extended at least up to $200 \mathrm{AU}$. Line ratios indicate $\mathrm{H}_{2}$ gas at $T>2000 \mathrm{~K}$, which implies the observed emission might be excited by energetic electrons from X-rays. The intensity of $\mathrm{H}_{2}$ 1-0 $\mathrm{S}(1)$ increases by an order of magnitude from the previous measurement carried out in 2003 by Bary et al. (2008), which indicates line variability.

\section{A.22. HD 97300}

HD 97300 (Sp. type: B9V; Keller et al. 2008) is one of the two intermediate-mass stars in Chamaeleon I (the other is HD 97048), which is one of the closest star-forming regions $(\sim 150 \mathrm{pc})$. A largely extended elliptical ring around HD $97300(\sim 7500 \mathrm{AU}$ or $0.045 \times 0.03 \mathrm{pc}$ in size) has been found in MIR bands (e.g., Siebenmorgen et al. 1998; Kóspál et al. 2012), of which the emission is considered to be dominated by PAH emission. The origin of the ring structure can be explained by a bubble blown into the surrounding interstellar matter and heated by the star (Kóspál et al. 2012). No IR-excess at $\lesssim 24 \mu \mathrm{m}$ is found, indicating that HD 97300 is close to a ZAMS (Kóspál et al. 2012).

\section{A.23. HD 98922}

HD 98922 is a young $(\lesssim 0.01 \mathrm{Myr}$, Manoj et al. 2006) Herbig Be star (B9Ve; Juhász et al. 2010), and its distance is rather uncertain (e.g., $~ 500-2000$ pc: Alecian et al. 2013; 
Maaskant et al. 2014; Hales et al. 2014). Like HD 95881, HD 98922 is classified as a group II source (i.e., self-shadowed disk) with the presence of PAH emission and [O I] line emission (Acke et al. 2005; Verhoeff et al. 2010). This implies that the [O I] emission might come from a gaseous disk inside the dust-sublimation radius (Acke et al. 2005). Recent NIR interferometric observations with VLTI/AMBER by Caratti o Garatti et al. (2015) indicate that the $\mathrm{Br} \gamma$-emitting region $(\sim 0.31 \pm 0.04 \mathrm{AU})$ is smaller than the continuum-emitting region (inner radius of the dust disk: $\sim 0.7 \pm 0.2 \mathrm{AU}$ ). Also, they have spatially resolved the circumstellar disk of HD 98922 with the VLT/SINFONI integral field spectrograph, which is extended in $K$-band up to $\sim 140 \mathrm{AU}$ in diameter at a distance of $440_{-50}^{+60} \mathrm{pc}$. Its MIR spectrum around $10 \mu \mathrm{m}$ shows a broader and flatter silicate feature than that typically seen in the ISM (van Boekel et al. 2003), indicating large (1-2 $\mu \mathrm{m})$ processed grains as well as minor spectral features associated with crystalline olivines and pyroxenes.

\section{A.24. HD 100453}

The Herbig Ae star HD 100453 (spectral type of A9Ve; Keller et al. 2008) is thought to be in transition from a gas-rich PPD to a gas-depleted debris disk (Collins et al. 2009). Its Spitzer/IRS spectrum shows a very weak sign of silicate features at 10 and $20 \mu \mathrm{m}$. This, together with SED analysis, indicates the presence of a gap in the disk (e.g., Mariñas et al. 2011; Maaskant et al. 2013; Khalafinejad et al. 2016). A spatially resolved MIR Q-band image obtained with Gemini north/MICHELLE indicates that the outer edge of the gap is at $20_{-3}^{+3} \mathrm{AU}$ and the disk is extended up to $\sim 200 \mathrm{AU}$ (Khalafinejad et al. 2016).

\section{A.25. HD 100546}

HD 100546 is one of the nearest Herbig Be stars (Sp. type: B9Ve, Acke \& van den Ancker 2004; $d \sim 103 \pm 6 \mathrm{pc}$, van Boekel et al. 2005). It is the first disk in which crystalline silicates (Hu et al. 1989) and PAHs (Malfeit et al. 1998) have been detected. Its 8.6 and $11.3 \mu \mathrm{m}$ PAH features are spatially extended on a few 100 AU scale (van Boekel et al. 2004), and the spatial distribution of the $3.3 \mu \mathrm{m}$ emission shows a gap in the innermost region $(R \sim 5-10 \mathrm{AU}$ ) and is extended up to $\sim 50 \mathrm{AU}$ (Habart et al. 2006) or $R \sim 12 \pm 3 \mathrm{AU}$ (Geers et al. 2007b). Minor features at 3.4 and $3.46 \mu \mathrm{m}$ are also detected (Habart et al. 2006), which could be attributed to aliphatic $\mathrm{C}-\mathrm{H}$ stretches in methyl or ethyl side-groups attached to PAHs (e.g., Joblin et al. 1996; Yang et al. 2013, 2016b). Lisse et al. (2007) compared the MIR spectrum (5-35 $\mu \mathrm{m})$ of HD 100546 with those of Comet 9P/Tempel 1 and Comet

C/ 1995 O1 (HaleBopp), which shows similar emission signatures including PAH features as 
well as crystalline silicate features. Similar to HD 97048, $\mathrm{H}_{2}$ 1-0 $\mathrm{S}(1)$ emission at $2.12 \mu \mathrm{m}$ is detected (Carmona et al. 2011), which may be extended at least up to $50 \mathrm{AU}$ from the central star. Based on HST/NICMOS2 coronagraphic observations at $1.6 \mu \mathrm{m}$, Augereau et al. (2001) detected an elliptical structure extended up to 350-380 AU and inferred that the disk is marginally optically thick inside $80 \mathrm{AU}$ and optically thin further out. A gas giant planet at $\sim 50 \mathrm{AU}$ from the central star has been confirmed by direct imaging (Quanz et al. 2015), and multiple planet formation is expected from the HD 100546 system (e.g., Currie et al. 2015; Pinilla et al. 2015; Quanz et al. 2015).

\section{A.26. HD 101412}

HD 101412 (a.k.a. CD-59 3865) is a Herbig Ae star (Sp. type of B9.5Ve-A0; Geers et al. 2006, Juhász et al. 2010). The distance to this star is rather uncertain (e.g., $d=600 \pm 100$ pc, Folsom et al. 2012; $d \sim 160$ pc, Juhász et al. 2010; $d \sim 118$ pc, Maaskant et al. 2015). Geers et al. (2007b) performed $N$-band and $L$-band observations toward HD 101412 using VISIR and ISAAC instruments at the VLT, which place an upper limit on the radial spatial extent of the 3.3, 8.6, and $11.2 \mu \mathrm{m}$ PAH features to be $\lesssim 38, \lesssim 26$, and $\lesssim 24$ AU, respectively at a distance of $160 \mathrm{pc}$. In the $N$-band spectrum, a weak and broad feature at $11.2 \mu \mathrm{m}$ confused with the $11.2 \mu \mathrm{m}$ PAH feature is confirmed, which is possibly due to crystalline silicates (Geers et al. 2006). Fedele et al. (2008) compared the spatial distribution of the gas traced by [O I] $6300 \AA$ with that of the dust traced by the $10 \mu \mathrm{m}$ emission in the HD 101412 disk, and found that the disk is strongly flared in gas but self-shadowed in dust beyond $\sim 2 \mathrm{AU}$. This might imply that the disk was initially flared then became flat by gas-dust decoupling, grain-growth, and dust settling.

\section{A.27. HD 135344B}

HD 135344B (spectral type of F4Ve; Dunkin et al. 1997), also known as SAO 206462, is located at a distance of $142 \mathrm{pc}$. With an age of $8_{-4}^{+8} \mathrm{Myr}$ (van Boekel et al. 2005), HD $135344 \mathrm{~B}$ is one of the most studied PPDs in detail. Its disk structure has been spatially resolved in multi-wavebands, which shows dust cavity, spiral structures, asymmetries in the outer disk (e.g., Pontoppidan et al. 2008; Brown et al. 2009; Muto et al. 2012; Pérez et al. 2014; Stolker et al. 2016). Its remarkable features include the existence of CO $4.7 \mu \mathrm{m}$ emission inside the dust cavity at 45 AU (Pontoppidan et al. 2008) and the NIR scattered light extending close to $25 \mathrm{AU}$ from the central star (e.g., Muto et al. 2012). Its NIR-excess indicates the existence of hot dust with a temperature of $\sim 1500 \mathrm{~K}$ (e.g., Coulson \& Walther 
1995). With the above characteristics, HD 135344B is considered to have a pre-transitional disk. Its Spitzer/IRS spectra show weak PAH features and no indication of silicate emission (e.g., Geers et al. 2006; Keller et al. 2008). The profile of its "8.0" $\mu \mathrm{m}$ feature is a blend of class B and C following the classification of Peeters et al. (2002). The $8.6 \mu \mathrm{m}$ PAH feature is marginally detected (Sloan et al. 2005). The $11.2 \mu \mathrm{m}$ feature is noticeably shifted to longer wavelengths and is relatively broader than that typically seen in the ISM (Sloan et al. 2005; Geers et al. 2006). Pech et al. (2002) claimed that the broadening of the $11.2 \mu \mathrm{m}$ feature could be due to anharmonicity, which might imply the presence of very hot PAHs in the innermost part of the HD 135344B disk (Geers et al. 2006).

\section{A.28. HD 139614}

HD 139614 (Sp. type: A7Ve; Keller et al. 2008) is considered to be a pre-transitional disk (e.g., Espaillat et al. 2008), which is characterized by NIR excess with weak or no silicate feature at $10 \mu \mathrm{m}$ (Juhász et al. 2010), indicating a gap separation between inner and outer disks. Based on MIR interferometric data obtained with VLTI/MIDI combined with SED analysis, Matter et al. (2014) suggest the presence of an optically thin inner disk at $0.22-$ 2.3 AU, a gap, and an outer disk from 5.6 AU. Later, with more comprehensive data, Matter et al. (2015) found that an interaction between a single giant planet $\left(\sim 3 M_{J}\right.$ at $\sim 4.5 \mathrm{AU}$ from the star) and the disk can induce the gap structure in warm $\mu \mathrm{m}$-sized dust distribution appearing at $\sim 2.5-6 \mathrm{AU}$.

\section{A.29. HD 141569}

The nearby $(d=116 \pm 8$ pc; Folsom et al. 2012) HAeBe star HD 141569 (Sp. type: B9.5 Ve; Juhász et al. 2010) is well-known to have a large ( $R \sim 400 \mathrm{AU})$, optically thin disk with two ring-like structures: an outer ring at $\sim 325 \mathrm{AU}$ with a width of $\sim 150 \mathrm{AU}$ and an inner ring at $\sim 200 \mathrm{AU}$ (e.g., Augereau et al. 1999; Weinberger et al. 1999; Mouillet et al. 2001). Later, more complex structures including outer spirals and an additional arc-like feature have been found (e.g., Biller et al. 2015). Either an interaction with two M-type companion stars (e.g., Augereau \& Papaloizou 2004) or planet formation (Wyatt 2005), or both, can account for the disk structures. Recent observations have further discovered an inner disk component inside the inner ring (e.g., Currie et al. 2016; Konishi et al. 2016), but no point-like source has been detected within the gap between the inner disk and the inner ring (Konishi et al. 2016). Li \& Lunine (2003) modeled the IR emission from the HD 141569 disk using a porous dust model together with a population of PAH molecules and 
found that PAHs in the disk are largely charged and dust grains with a high porosity are preferred. The PAH parameters derived in this work $\left(\left\langle a_{0}\right\rangle=3.0 \pm 1.0 \AA,\langle\sigma\rangle=0.3 \pm 0.1\right.$, and $\left.\left\langle\phi_{\text {ion }}\right\rangle=0.9 \pm 0.1\right)$ are in good agreement with their results $\left(a_{0} \approx 2.5 \AA\right.$ and $\left.\sigma \approx 0.3\right)$.

\section{A.30. HD 142527}

HD 142527 is a Herbig Fe star (Sp. type: F7IIIe; Acke \& van den Ancker 2004) located in a distance of $\sim 145$ pc (e.g., Acke \& van den Ancker 2004; Fukagawa et al. 2010) or $\sim 200$ pc (e.g., Alonso-Albi et al. 2009; Maaskant et al. 2014). Its MIR (18.8 and $24.5 \mu \mathrm{m}$ ) images exhibit an inner disk $(R \sim 5 \mathrm{AU})$, an outer disk with bright arc-like emission, and a wide gap devoid of dust (and gas) between $\sim 30$ and $90 \mathrm{AU}$ (Fujiwara et al. 2006). Recent ALMA observations have revealed the detailed disk structures (e.g., Casassus et al. 2013, 2015; Fukagawa et al. 2013; Muto et al. 2015) and flows of gas through the dust depleted gap (Casassus et al. 2013). It is found that there is a low-mass companion (HD 142517B) with a stellar mass of $\sim 0.1-0.4 M_{\odot}$ at $\approx 12 \mathrm{AU}$ from the central star (e.g., Biller et al. 2012; Rodigas et al. 2014; Lacour et al. 2016), which may have played a role in generating the large gap in the disk of the HD 142527 system.

\section{A.31. HD 142666}

HD 142666 (a.k.a. V1026 Sco) is a Herbig Ae star (Sp. type: A8Ve; Keller et al. 2008) located in the Sco OB2-2 association. Its group II type SED (Meeus et al. 2001) and the presence of PAH emission imply that the HD 142666 disk could be in transition between a gas-rich flaring disk to a gas-poor self-shadowed disk (e.g., Verhoeff et al. 2010). This object is reported to have $\operatorname{Br} \gamma$ emission (e.g., Eisner et al. 2009). Dent et al. (2005) detected CO emission, predicting a nearly face-on disk with a radius of $\sim 45 \mathrm{AU}$. Based on the SCUBA 450 and $850 \mu \mathrm{m}$ images, Sandell et al. (2011) reported the disk size to be $\lesssim 2$ '.2 (i.e., $\lesssim 330 \mathrm{AU}$ at $d \sim 145 \mathrm{pc})$. van Boekel et al. (2003) found that the mass ratio of large $(2.0 \mu \mathrm{m})$ to small $(0.1 \mu \mathrm{m})$ grains is larger than unity $(\sim 1.54)$ and interpreted this as the removal of small grains from the disk by radiation pressure. Using NIR imaging polarimetry, Hales et al. (2005) reported a large degree of polarization $(P \approx 1.32 \%$ in $J$-band $)$, which is intrinsic to the system. 


\section{A.32. HD 144432}

HD 144432 is a Herbig Ae star (Sp. type: A9Ve; Keller et al. 2008) located in the Sco OB2-2 association at a distance of $145 \pm 43$ pc (e.g., van Boekel et al. 2005) or $d \sim 253 \mathrm{pc}$ (Keller et al. 2008). Distinctive P Cyg profiles are seen in its optical spectrum, indicating extensive stellar activity in the HD 144432 environment (Dunkin et al. 1997). The HD 144432 disk has been spatially resolved in MIR (Leinert et al. 2004), and its size is measured to be $0^{\prime \prime} 014$ corresponding to $2.1 \pm 0.2 \mathrm{AU}$ at $145 \mathrm{pc}$. HD 144432 exhibits strong silicate emission in its IR spectrum (e.g., Keller et al. 2008), which is one of the strongest observed in HAeBe stars (van Boekel et al. 2003).

\section{A.33. HD 145718}

The Herbig Ae star HD 145718 (a.k.a. V718 Sco) has a spectral type of A8III/IVe (e.g., Keller et al. 2008) or A5Ve (Carmona et al. 2010). It is likely associated with the $\rho$ Ophiuchus cloud. It is a photometric variable with a small variation (e.g., $\sim 1 \%$ in V), and its binarity is uncertain (e.g., Friedemann et al. 1996). Guimarães et al. (2006) detected redshifted absorption features only in the Balmer lines, indicating circumstellar activity with hydrogen-rich material. Dent et al. (2005) estimated a disk radius of $\sim 60 \pm 30 \mathrm{AU}$ from the $J=3-2{ }^{12} \mathrm{CO}$ emission.

\section{A.34. HD 163296}

HD 163296 (a.k.a. MWC 275) is a young ( 5 Myr; e.g., van den Ancker et al. 1998; Alecian et al. 2013) Herbig Ae star (Sp. type: A1Ve; Juhász et al. 2010). The disk around HD 163296 has been spatially resolved (e.g., Grady et al. 2000; Fukagawa et al. 2010; Sandell et al. 2011; Garufi et al. 2014), which is extended to $440 \mathrm{AU}$ and shows a broken ring structure. Asymmetric supersonic jets associated with HD 163296 are found, which are roughly perpendicular to the disk plane (e.g., Devine et al. 2000; Wassell et al. 2006). Based on its ISO spectra, van den Ancker et al. (2000) reported that the IR fluxes of HD 163296 are dominated by solid state features. The detection of a minor feature at $6.85 \mu \mathrm{m}$ attributed to aliphatic hydrocarbons has been reported (Bouwman et al. 2001), and a nanodiamond feature at $3.53 \mu \mathrm{m}$ is tentatively detected by Acke \& van den Ancker (2006). van den Ancker et al. (2000) also pointed out the presence of a population of very large, mm-sized grains in the HD 163296 disk. Recent observations with ALMA and VLA indicate a population of large grains and pebbles $(\gtrsim 1 \mathrm{~cm})$ in the inner region (i.e., $\lesssim 50 \mathrm{AU}$ ) of the disk and the 
presence of small grains similar to those in the diffuse ISM in the outer (i.e., $\gtrsim 150 \mathrm{AU}$ ) disk (Guidi et al. 2016). Since the CO snowline at $~ 90$ AU has already been identified by ALMA (Qi et al. 2015), the different populations of dust grains along the radial distance could imply grain growth at the CO snowline and transportation to the inner regions (Guidi et al. 2016).

\section{A.35. HD 169142}

HD 169142 (a.k.a. MWC 925, SAO 186777) is a Herbig Ae star (A5Ve; Keller et al. 2008) showing complex disk structures. At least two cavities, one ring structure, and an outer disk have been spatially resolved (e.g., Honda et al. 2012; Quanz et al. 2013; Osorio et al. 2014; Momose et al. 2015). In addition, its SED shows a considerable NIR (2-6 $\mu \mathrm{m})$ excess, suggesting that the disk is pre-transitional (i.e., the presence of an inner disk; Espaillat et al. 2007). Recent observations revealed a point-like feature within the inner cavity (Biller et al. 2014; Reggiani et al. 2014), implying (multiple) planet formation in the disk. Seok \& Li (2016) performed a comprehensive modeling of its SED as well as the PAH emission features with porous dust and astronomical-PAHs and found that three dust populations and relatively small PAH molecules $\left(a_{0}=3.0 \AA\right.$ and $\left.\sigma=0.4\right)$ with an ionization fraction of $\phi_{\text {ion }}=0.6$ can explain the entire SED and the observed PAH features. These results are consistent with those derived in this work $\left(\left\langle a_{0}\right\rangle=3.0 \pm 0.5 \AA,\langle\sigma\rangle=0.3 \pm 0.1\right.$, and $\left.\left\langle\phi_{\text {ion }}\right\rangle=0.5 \pm 0.2\right)$.

\section{A.36. HD 179218}

HD 179218 (a.k.a. MWC 614) is a young ( 1 Myr; e.g., van Boekel et al. 2005; Alecian et al. 2013) isolated HAeBe star with a spectral type of B9e (Acke \& van den Ancker 2004). As its IR spectra are dominated by crystalline forsterite and enstatite rather than small, amorphous silicate grains (e.g., Bouwman et al. 2001; Schütz et al. 2005; van Boekel et al. 2005), HD 179218 is known to have the second largest percentage of crystalline dust (van Boekel et al. 2005). This indicates dust processing in the circumstellar disk of HD 179218, and the presence of cold enstatite at $\gtrsim 10 \mathrm{AU}$ implies that enstatite is mostly produced in the inner regions and is transported outward by radial mixing (van Boekel et al. 2005). A double-ring-like emission at $\sim 10 \mu \mathrm{m}$ has been spatially resolved (e.g., Fedele et al. 2008), peaking at $\sim 1$ and $20 \mathrm{AU}$, respectively. In addition, a gap at $\sim 10 \mathrm{AU}$ is reported based

on the MIR interferometry with VLTI/MIDI (Menu et al. 2015). [O I] $6300 \AA$ line emission tracing the distribution of the gas in HD 179218 has been observed (e.g., Fedele et al. 2008; 
van der Plas et al. 2008), which suggests a flaring disk structure in HD 179218. The [O I] line emission is probably extended to $\sim 65 \mathrm{AU}$ from the central star (van der Plas et al. 2008) but is mainly produced between $\sim 1-10$ AU (Fedele et al. 2008).

\section{A.37. HD 200775}

HD 200775 (a.k.a. MWC 361) is a young, massive spectroscopic binary $(\sim 0.1 \mathrm{Myr}$, $\sim 10 M_{\odot}$ for both components; e.g., Alecian et al. 2008). It illuminates the reflection nebula NGC 7023 which is well-known to show strong PAH emission. Its distance is commonly adopted to be $\sim 430$ pc (e.g., Acke \& van den Ancker 2004; Alecian et al. 2013), but more recently, Benisty et al. (2013) claimed $d=320 \pm 51$ pc based on new radial velocity measurements. A biconical cavity associated with HD 200775 has been observed with CO and HI observations (Fuente et al. 1998), which is probably due to an energetic, bipolar outflow during its earlier evolutionary stage. Okamoto et al. (2009) found elongated emission extended in $\sim 1000$ AU scale in MIR, perpendicular to the cavity wall, which can be explained by an inclined flared disk. They also reported an amorphous silicate feature peaking at $9.2 \mu \mathrm{m}$, which is substantially shifted from the typical wavelength of $\sim 9.5-9.8 \mu \mathrm{m}$ where the amorphous silicate features seen in the diffuse ISM and other PPDs typically peak. This might suggest alternation of grains due to plasma irradiation. HD 200775 displays a dipolar

magnetic field geometry with similar characteristics to the magnetic fields of Ap/Bp stars (e.g., Alecian et al. 2008).

\section{A.38. HD 244604}

HD 244604 (a.k.a. V1410 Ori) is a Herbig Ae star (Sp. type: A0-3; e.g., Marinãs et al. 2011). Depending on the assumed extinction $\left(A_{V} \sim 0.14-0.57 \mathrm{mag}\right)$, its stellar luminosity and age are estimated to be in the range of $\sim 22-55 L_{\odot}$ and $\sim 1.9-8 \mathrm{Myr}$, respectively (e.g., Marinãs et al. 2011; Alecian et al. 2013; Fairlamb et al. 2015; Kama et al. 2015). We adopt $L_{\star}=55 L_{\odot}$ and $\tau=2.79 \mathrm{Myr}$ from Alecian et al. (2013). Its IRS spectrum shows strong silicate emission with weak PAH features. The subtraction of the strong $9.7 \mu \mathrm{m}$ silicate feature results in a substantial non-PAH residual in the $7-12 \mu \mathrm{m}$ range (see Figure 5). Folsom et al. (2012) examined the chemical abundances of HD 244604, which show no selective depletion or enhancement. 


\section{A.39. HD 250550}

HD 250550 (a.k.a. MWC 789, V1307 Ori) is a young ( 1 Myr; e.g., see Liu et al. 2011, Fairlamb et al. 2015) Herbig Be star with a spectral type of B4-5IIIe (e.g., Juhász et al. 2010). Its distance is commonly adopted to be $\sim 700$ pc (e.g., Catala et al. 1991; Bouret et al. 2003; Juhász et al. 2010), but a much shorter distance $(\sim 280$ pc $)$ also has been listed in the literature (e.g., Maaskant et al. 2014; Hein Bertelsen et al. 2016). Catala et al. (1991) observed short-term variations in CaII K line, indicative of rotational modulation seen in $\mathrm{AB}$ Aur or HD 163296. Using far-UV spectroscopy, Bouret et al. (2003) detected C III and O IV emission lines, revealing very high temperature regions $\left(\approx 3 \times 10^{5} \mathrm{~K}\right)$ around the star. This would suggest the existence of a circumstellar halo (e.g., Leinert et al. 2001). Hein Bertelsen et al. (2016) reported a detection of broad CO line emission from the HD 250550 disk and

the FWHM of the CO lines increases with $J$, the rotational quantum number, suggesting a radially extended $\mathrm{CO}$-emitting region.

\section{A.40. HD 259431}

HD 259431 (a.k.a. V700 Mon, MWC 147) is a Herbig Be star (Sp. type: B1Ve-B6e; Juhász et al. 2010; Sandell et al. 2011; Li et al. 2014) associated with the Mon R1 association, and illuminates the reflection nebula NGC 2247. Its distance is estimated to vary from $\sim 290$ pc (e.g., Juhász et al. 2010) to 800 pc (Kraus et al. 2008b). Depending on the adopted distance, its stellar luminosity also largely varies (e.g., $\approx 320 L_{\odot}: \mathrm{Li}$ et al. $2014 ; 760_{-350}^{+500} L_{\odot}$ : Menu et al. 2015; $1550 L_{\odot}$ : Kraus et al. 2008b; $2233 L_{\odot}$ : Alecian et al. 2013). Li et al. (2014) found similarities between HD 259431 and MWC 1080, suggesting that the extended, filamentary emission at 11.2 and $18.5 \mu \mathrm{m}$ surrounding HD 259431 may arise from an outflow cavity like MWC 1080. The CO first overtone bandhead emission of HD 259431 has been detected (Ilee et al. 2014), which extends from 0.89 to $4.3 \mathrm{AU}$ at an inclination of $52^{\circ}$. This inclination is in good agreement with that measured from $K$-band spectrointerferometry with VLTI/AMBER by Kraus et al. (2008b). Kraus et al. also spatially resolved NIR $(2.2 \mu \mathrm{m})$ and MIR $(11 \mu \mathrm{m})$ emission from HD 259431, which are extended to $\sim 1.3$ and 9 AU, respectively. They conclude that the NIR emission is dominated by accretion luminosity from an optically thick inner gaseous disk whereas the MIR emission is attributed to the outer dust disk. 


\section{A.41. HD 281789}

HD 281789 (a.k.a. F04101+3103) is a HAeBe star with a spectral type of A0-1 (e.g., Furlan et al. 2006; Keller et al. 2008). This object is not well studied in the literature, but it is included in a survey of TTSs in the Taurus star-forming regions (Furlan et al. 2006). Following the SED group classification of Meeus et al. (2001), Keller et al. (2008) classified the HD 281789 disk as a group IIa member (i.e., self-shadowed disk).

\section{A.42. IC348 LRL110}

IC348 LRL110 (Sp. type: M0.5, $T_{\text {eff }} \approx 3778 \mathrm{~K}$; Merín et al. 2010) is one of the coldest objects in our sample that shows clear detection of PAH features (see Figure 5), supporting that the excitation of PAHs does not require UV photons (Li \& Draine 2002, Mattioda et al. 2005). This object is associated with the Perseus star-forming region located at $\sim 250 \mathrm{pc}$ and is photometrically selected as a candidate for cold disks (i.e., disks with large inner dust holes) based on the "Cores to Disks" (c2d) Spitzer survey data (Evans et al. 2009). Merín et al. (2010) performed follow-up IRS observations toward IC348 LRL110 and found the presence of PAH emission as well as $9.7 \mu \mathrm{m}$ silicate emission but no hint for a hole inside its disk.

\section{A.43. IC348 LRL190}

Similar to IC348 LRL110, IC348 LRL190 (Sp. type: M3.75, $T_{\text {eff }} \approx 3306 \mathrm{~K}$; Merín et al. $2010)$ is associated with the Perseus star-forming region $(d \sim 250 \mathrm{pc})$ and is included in the c2d survey. This object is suggested to have a hole with a radius of $\sim 5 \pm 1 \mathrm{AU}$ (Merín et

al. 2010; or $R \sim 2-4 \mathrm{AU}$; van der Marel et al. 2016). Its IRS spectrum is rather noisy and dominated by silicate emission (see Figure 9), so it is excluded in our model calculation. The presence of PAH features in this very cold disk (even colder than IC348 LRL110) is reported in Merín et al. (2010, see their Table 8), probably based on the broad feature shown at $6.2 \mu \mathrm{m}$.

\section{A.44. IRAS $03260+3111$}

IRAS $03260+3111$ is a young stellar object in the NGC 1333 molecular cloud core (a.k.a. NGC 1333 SVS 3) and has a binary system (Sp. type: B5+F2; Kóspál et al. 2012) 
with a wide separation of $\sim 1160 \mathrm{AU}$ (i.e., 3'.62) assuming a distance of $d=320 \mathrm{pc}$ to Perseus (Herbig 1998). This object shows a prototypical PAH spectrum of class A defined by Peeters et al. (2002, see Figure 6 of this paper and Figure 5 of Sloan et al. 2005). Spatial variations in the strength and shape of the PAH features with increasing distance from the central star have been noticed (e.g., Sloan et al. 1999). In particular, an excess at $\sim 10.8-11.0 \mu \mathrm{m}$ beside the $11.2 \mu \mathrm{m}$ PAH feature and a feature seen at $\sim 10 \mu \mathrm{m}$ disappear further from the central star (Sloan et al. 1999) similar to the trend that the fraction of PAH cations decreases with increasing distance (Joblin et al. 1996). This indicates these features are associated with PAH cations. Haisch et al. (2006) suggested that the primary is a Class II based on the MIR photometry and the secondary is at least a Class II YSO based on their upper limits of the $10 \mu \mathrm{m}$ fluxes.

\section{A.45. IRAS 06084-0611}

IRAS 06084-0611 is a star-forming region (also known as GGD 12-15) embedded in the Monoceros molecular cloud at a distance of $\sim 1050$ pc (Boersma et al. 2009) or $830 \pm 50$ pc (Maaskant et al. 2011). Two very bright, massive stars have been detected (e.g., Persi \& Tapia 2003; Maaskant et al. 2011): a cometary HiI region VLA1 (IRS4) and a Herbig Be star VLA4 (IRS2) with a spectral type of B3 (Boersma et al. 2009). VLA4 shows the PAH emission features in its MIR spectrum obtained with the TIMMI2 camera mounted at the ESO $3.6 \mathrm{~m}$ telescope (e.g., Boersma et al. 2009). The $11.2 \mu \mathrm{m}$ PAH emission is spatially resolved (FWHM $\sim 2$ '.2 or $2200 \mathrm{AU})$, which is more extended than the nearby $(10 \mu \mathrm{m})$ continuum (FWHM 1".2 or $1300 \mathrm{AU})$. Considering the large extent of the PAH emission, it is most likely that the surrounding materials around VLA4 dominate the observed PAH emission.

\section{A.46. J032903.9+305630}

SSTc2d J032903.9+3056 is a TTS (Sp. type: G7; Merín et al. 2010), which is associated with the Perseus star-forming region. Its IRS spectrum shows silicate absorption as well as water absorption features indicating that it is close to an edge-on system (Merín et al. 2010). Although the broad features at 7-9 $\mathrm{m}$ are likely due to PAH emission (see Figure 9), other PAH features are not clearly detected, so we exclude this object for model calculation. 


\section{A.47. J182858.1+001724}

SSTc2d J182858.1+001724 is a TTS (Sp. type: G3; Merín et al. 2010) and is associated with the Serpens star-forming region $(d \sim 260 \mathrm{pc})$. This is one of the coldest objects $\left(T_{\text {eff }} \sim\right.$ $5830 \mathrm{~K}$; Merín et al. 2010) in our sample with PAH-dominated spectra (see Figure 6). J182858.1+001724 shows strong, extended emission in the MIPS $70 \mu \mathrm{m}$ band similar to that of DoAr 21 (Merín et al. 2010), which is likely due to contamination from the surrounding materials.

\section{A.48. J182907.0+003838}

SSTc2d J182907.0+003838 is a TTS associated with the Serpens star-forming region (Sp. type: K7; Merín et al. 2010). Its IRS spectrum is dominated by silicate emission and shows marginal detection of PAH features (see Figure 9, and also see Table 8 in Merín et al. 2010). It does not show any hint for a hole in the disk.

\section{A.49. $\mathrm{LkH} \alpha 224$}

LkH $\alpha 224$ (a.k.a. V1686 Cyg) is a HAeBe star and belongs to a small pre-main-sequence star cluster with $\mathrm{BD}+40^{\circ} 4124$ and $\mathrm{LkH} \alpha 225$ (e.g., van den Ancker et al. 2000; Sandell et al. 2011). Its nature reported in the literature is rather uncertain; its spectral type is reported as B2-3e (e.g., Juhász et al. 2010), B5 (e.g., Alonso-Albi et al. 2009), A7e (e.g., Acke \& van den Ancker 2004), and F9 (e.g., Manoj et al. 2006). Similarly, its effective temperature ranges between $\sim 6170$ and $15500 \mathrm{~K}$ (e.g., Acke \& van den Ancker 2004; Manoj et al. 2006; Alonso-Albi et al. 2009). LkH $\alpha 224$ has characteristics of UX Ori type stars such as optical

variations with $\gtrsim 1 \mathrm{mag}$ on timescales of days to weeks. The IR spectra obtained with $I S O$ are examined by van den Ancker et al. (2000) in detail. IR emission lines detected in the IR spectra can be explained by a single non-dissociated shock, and the shock might be produced by a slow outflow from $\mathrm{LkH} \alpha 225$.

\section{A.50. $\quad \operatorname{LkH} \alpha 330$}

LkH $\alpha 330$ (a.k.a. F03426+3214) is a classical TTS (Sp. type: G3e; Geers et al. 2006) associated with the Perseus molecular clouds. Brown et al. (2008) carried out $340 \mathrm{GHz}$ $(880 \mu \mathrm{m})$ dust continuum imaging with the Submillimeter Array (SMA) and resolved the 
disk with an inner hole $(r \sim 40 \mathrm{AU})$ devoid of dust but gas still remaining. A steep transition between the hole and the outer disk is found, which could be explained by the truncation of the outer disk via gravitational instabilities (e.g., Brown et al. 2008). Andrews et al. (2011) also measured the cavity size with new SMA data, giving a slightly larger radius ( $68 \mathrm{AU})$. The $1.3 \mathrm{~mm}$ observations with the Combined Array for Research in Millimeterwave Astronomy (CARMA) revealed that a lopsided ring at $\sim 100 \mathrm{AU}$ in the $\mathrm{LkH} \alpha 330$ disk (Isella et al. 2013). While the 6.2 and $11.2 \mu \mathrm{m}$ PAH features are clearly shown in the Spitzer/IRS spectrum (see Figure 6), the 7.7 and $8.6 \mu \mathrm{m}$ features, often coming together with the $6.2 \mu \mathrm{m}$ feature are only marginally detected. This might be affected by the disk geometry such as disk thickness (Geers et al. 2006), or more likely, hidden by the strong silicate emission feature at $9.7 \mu \mathrm{m}$.

\section{A.51. MWC 297}

MWC 297 (a.k.a. NZ Ser, PDS 518) is a B1.5Ve type Herbig Be star (e.g., Acke \& van den Ancker 2004). It is one of the closest massive young stars $(d \sim 250$ pc; e.g., Acke et al. 2008). The $\operatorname{Br} \gamma$ line and continuum emission measurements of MWC 297 by Malbet et al. (2007) suggest a flat, optically thick accretion disk with an outflowing wind around MWC 297. The regions emitting the continuum emission are much more compact than the dust sublimation radius expected for an irradiated dust disk, and the line-emitting region is more extended than the continuum-emitting region (e.g., Kraus et al. 2008a). Using interferometric spectrographs, AMBER and MIDI mounted on the VLTI, Acke et al. (2008) have spatially resolved the IR emission including the $9.7 \mu \mathrm{m}$ silicate feature and found that all these emissions originate from a very compact region $(\mathrm{FWHM}<1.5 \mathrm{AU})$ with no evidence for an inner emission-free gap. To explain the observed IR emission, Acke et al. (2008) adopted a geometric model consisting of three Gaussian disks, which can account for the IR emission but underestimates the observed submm/mm emission. Verhoeff et al. (2012) have marginally resolved the PAH emission in the narrow-band peaking at $11.88 \mu \mathrm{m}$ with VLT/VISIR, constraining a spatial extension of $\sim 110 \mathrm{AU}$ in diameter. The mm observations obtained with the VLA and the IRAM Plateau de Bure interferometers (Alonso-Albi et al. 2009) also showed that the MWC 297 disk is small, with an outer radius of $\sim 28 \mathrm{AU}$. NIR polarization produced by multiple scattering has been observed (Pereyra et al. 2009), supporting the presence of an optically thick disk. 


\section{A.52. MWC 865}

MWC 865 (a.k.a. V921 Sco, CD-42 11721, Hen 3-1300) is a Herbig Be star with a spectral type of B0IVep (e.g., Acke \& van den Ancker 2004), embedded in a small, dark cloud (Boersma et al. 2009) as the most massive member of a small star cluster (e.g., Weidner et al. 2010). Its distance is rather uncertain, ranging from $\sim 160 \mathrm{pc}$ to $2.2 \mathrm{kpc}$ (e.g., Kreplin et al. 2011 and references therein), thus other stellar parameters are also not well-constrained (e.g., $T_{\text {eff }} \approx 14000 \pm 1000 \mathrm{~K}$, Borges Fernandes et al. 2007; $T_{\text {eff }} \approx 30000 \mathrm{~K}$, Acke \& van den Ancker 2004). While the ISO spectrum is dominated by strong PAH emission, the IR spectra obtained with ground-based telescopes do not show any evidence of PAH features but exhibit strong HI lines such as Pf $\delta$ at $3.297 \mu \mathrm{m}$ and the Humphreys series (Acke \& van den Ancker 2006). The discrepancy can be explained by different aperture sizes, which indicates that the PAH emission does not originate from a confined region but is rather extended, possibly from the surrounding nebulosity (e.g., Acke \& van den Ancker 2004; Kraus et al. 2008a). Boersma et al. (2009) have studied the spatial distribution of the MIR emission features around MWC 865 and reported that an arc and a patch with an extent (FWHM) of $\sim 1^{\prime \prime}$ and $\sim 5^{\prime \prime}$ (i.e., 400 and $2000 \mathrm{AU}$ at $d \sim 400 \mathrm{pc}$ ) apart from the central star, respectively, show strong PAH emission beside one bright peak near the star. A tentative detection of the nanodiamond feature around $3.53 \mu \mathrm{m}$ is also reported (Acke \& van den Ancker 2006). The extinction along the line of sight toward MWC 865 is strong (e.g., $A_{V}=5.08$ mag; Acke \& van den Ancker 2004), and its SED is dominated by thermal emission of circumstellar dust. This, together with the detection of the HI emission lines, suggests that MWC 865 is most likely surrounded by an accretion disk.

\section{A.53. MWC 1080}

MWC 1080 (a.k.a. V628 Cas) is a young ( $\lesssim 1$ Myr) triple system associated with a small cluster embedded in the dark cloud LDN 1238 at a distance of $\sim 2.2 \mathrm{kpc}$ (e.g., Acke \& van den Ancker 2004; Alecian et al. 2013). Note that Eisner et al. (2003) claimed that the distance to MWC 1080 is $\sim 1 \pm 0.2 \mathrm{kpc}$ (Hillenbrand et al. 1992). The primary, MWC 1080A (Sp. type: B0e; Acke \& van den Ancker 2004), itself is an eclipsing binary (Shevchenko et al. 1994), and the secondary, MWC 1080B, is also a HAeBe star (Leinert et al. 1997). $\mathrm{CO}$ and $\mathrm{CS}$ observations revealed a biconical outflow cavity with a size of $0.3 \mathrm{pc} \times 0.05 \mathrm{pc}$ around MWC 1080, which was most likely created by the bipolar outflow from MWC 1080A (Wang et al. 2008). Using narrow-band MIR imaging at 11.2, 11.6, and $18.5 \mu \mathrm{m}$, Li et al. (2014) showed that the filamentary nebulosity of the MWC 1080 system extends to $\sim 0.15 \mathrm{pc}$, which traces the internal surfaces of the gas cavity. Strong extended emission at $850 \mu \mathrm{m}$ 
surrounding MWC 1080 is detected (Sandell et al. 2011), and a faint emission peak coincides with MWC 1080A. With $N$-band $(8-13 \mu \mathrm{m})$ spectra of the MWC 1080 system, Sakon et al. (2006) recognized a weak feature around $10.95-11.1 \mu \mathrm{m}$ (i.e., $11.0 \mu \mathrm{m}$ feature) attributed to a solo CH out-of-plane wagging mode of cationic PAHs (Hudgins \& Allamandola 1999). They found that the ratio of the $11.0 \mu \mathrm{m}$ feature to the $11.2 \mu \mathrm{m}$ PAH feature increases toward the central star and interpreted this as the promotion of PAHs' ionization to cationic species in the vicinity of the central star.

\section{A.54. Oph IRS48}

Oph IRS48 (a.k.a. WLY 2-48), a Herbig Ae star (Sp. type: A0; e.g., Brown et al. 2012a) is well-known to have a transitional disk, which is characterized by a gap in the disk. MIR imaging $(18.7 \mu \mathrm{m})$ has spatially resolved the dust disk around Oph IRS48, peaking at $\sim 55 \mathrm{AU}$ from the central star with a gap of $\sim 30 \mathrm{AU}$ in radius (Geers et al. 2007a). Its gas distribution traced by the $4.7 \mu \mathrm{m} \mathrm{CO}$ fundamental rovibrational band also confirms a hole at $\sim 30 \mathrm{AU}$ (Brown et al. 2012a). van der Marel et al. (2013) reported a highly asymmetric structure associated with large, mm-sized grains by ALMA observations, deviating from the MIR emission tracing small ( $\mu \mathrm{m}$-sized) grains. This is attributed to a dust trap, which would be the start of core formation (i.e., planet formation) near the central star (e.g., van der Marel et al. 2015). The radius of the hole revealed by mm-sized dust is measured to $\mathrm{be} \approx 13 \mathrm{AU}$, even inside the gas hole (Brown et al. 2012b). Its IRS spectrum shows strong PAH emission (see Figure 7), dominated by ionized PAHs $\left(\phi_{\text {ion }}=0.8\right)$. Maaskant et al. (2014) explained that highly ionized PAHs located in optically thin regions inside the disk cavity overwhelm the IRS spectrum whereas neutral PAHs from the outer disk contribute insignificantly. This is consistent with the size of the resolved PAH-emitting region being smaller than the neighboring dust continuum-emitting region (Geers et al. 2007b). As Oph IRS48 resides in a region with high extinction (e.g., $A_{V} \sim 11-12$ mag; Brown et al. 2012a, Follette et al. 2015), its stellar parameters such as $L_{\star}$ and age are not well-constrained. While Brown et al. (2012a) derived $\tau \sim 15 \mathrm{Myr}$ and $L_{\star} \sim 14.3 L_{\odot}$, Follette et al. (2015), adopting a much grayer extinction curve, estimated $\tau \sim 5-8 \mathrm{Myr}$ and a much higher $L_{\star}\left(\sim 24-45 L_{\odot}\right)$. The latter is more closer to the median age of the Ophiuchus members $(\sim 2-5 \mathrm{Myr})$.

\section{A.55. PDS $144 \mathrm{~N}$}

PDS 144 (a.k.a. CD-25 11111, IRAS 15462-2551) is a binary system consisting of two Herbig Ae stars (PDS $144 \mathrm{~N} \& \mathrm{~S}$ ) with a separation of 5".5 on the sky (e.g., Torres et al. 
1995), which is the first confirmed Herbig Ae-Herbig Ae wide binary (e.g., Hornbeck et al. 2012). The distance to PDS 144 is rather uncertain: while $\sim 1 \mathrm{kpc}$ is generally adopted (e.g., Perrin et al. 2006; Fairlamb et al. 2015), Hornbeck et al. (2015) claimed that its distance is $145 \pm 2$ pc considering PDS $144 \mathrm{~N} \& \mathrm{~S}$ are members of the Upper Sco association. An optically thick edge-on disk around the primary, PDS 144N (Sp. type: A2IV) has been spatially resolved (Perrin et al. 2006), and a dark lane of the edge-on disk is 0 '!15 in height and 0.8 in across. Prominent PAH emission is detected in PDS 144N (e.g., Perrin et al. 2006; Schütz et al. 2009), whereas the disk of the secondary, PDS 144S (Sp. type: A5V), lacks PAH emission. PDS 144N \& S are found to have intrinsic polarization, which is aligned with the local magnetic field and the jet axis (Pereyra et al. 2012), and the high NIR polarization of the PDS 144N disk is in favor of the presence of small grains similar to those of the diffuse ISM. Schütz et al. (2009) found that the spectral profile of the $8.6 \mu \mathrm{m}$ PAH feature is similar to that of PAHs in the ISM (i.e., PAH class A as determined by Peeters et al. 2002), whereas the peak wavelength of the $11.2 \mu \mathrm{m}$ PAH feature is red-shifted compared to those of class A and B profiles.

\section{A.56. RR Tau}

RR Tau is a young ( 1 Myr; e.g., Alonso-Albi et al. 2009), highly variable Herbig Ae star (Sp. type: A0IVev; e.g., Juhász et al. 2010). It is one of the UX Ori type stars showing an anti-correlation between the linear polarization and the brightness variation like BF Ori or WW Vul (e.g., Grinin et al. 1996; Rostopchina et al. 1997; Rodgers et al. 2002; Bedell et al. 201). Although we adopt a distance of $\sim 160 \mathrm{pc}$ and a luminosity of $L_{\star}=2 L_{\odot}$ from Acke $\&$ van den Ancker (2004), they are highly uncertain and sensitive to the assumed extinction. Larger distances $(d \sim 600-2100$ pc, Blondel \& Djie 2006, Alonso-Albi et al. 2009, Montesinos et al. 2009) and much higher luminosity (e.g., $L_{\star}=150 L_{\odot}$ at $d=800$ pc, Alonso-Albi et al. 2009) also have been reported in the literature. Using mm interferometric observations, Boissier et al. (2011) detected a disk around RR Tau with a size of $\sim 720 \mathrm{AU}$ (assuming $d=800 \mathrm{pc}$ ), which is much larger than the outer disk radius of $\sim 80 \mathrm{AU}$ derived from fitting the SED. This is possibly due to a complex geometry in the outer disk such as spiral arms or a secondary ring.

\section{A.57. RXJ1615.3-3255}

RXJ1615.3-3255 is a TTS (Sp. type: K4; Merín et al. 2010) associated with the Ophiuchus star-forming region at $\sim 120$ pc (or 185 pc; van der Marel et al. 2016). Its IRS spectrum 
as well as SED indicates the existence of a hole in its disk ( $R \approx 2 \pm 1$ AU; Merín et al. 2010), and millimeter imaging suggets a slightly large gap $\left(R=10_{-2}^{+10} \mathrm{AU}\right.$ : van der Marel et al. 2016, or $\sim 30 \mathrm{AU}$ : Andrews et al. 2011). Its IRS spectrum dominated by silicate emission is noisy and shows a marginal detection of the $11.2 \mu \mathrm{m}$ PAH feature possibly contaminated by the $11.2 \mu \mathrm{m}$ crystalline forsterite feature (see Figure 9 ).

\section{A.58. SR 21N}

SR 21N (a.k.a. Elias 2-30) is a young ( 1-3 Myr; e.g., Prato et al. 2003; Brown et al. 2009) TTS binary system located at the $\rho$ Ophiuchus cloud. The primary and secondary, separated by $\sim 6$ 6. 4 , have spectral types of G2.5 and M4, respectively (e.g., Geers et al. 2006). The disk around SR $21 \mathrm{~N}$ is known to be transitional; its cavity ( $R \approx 33-36 \mathrm{AU}$ ) has been spatially resolved (e.g., Brown et al. 2009; Andrews et al. 2011; Pérez et al. 2014), and the outer disk is extended to $\approx 140 \mathrm{AU}$ as revealed by the SMA $340 \mathrm{GHz}(880 \mu \mathrm{m})$ imaging. Inside the cavity, $\mathrm{SR} 21 \mathrm{~N}$ shows detectable warm $\mathrm{CO}$ emission arising from the inner regions of the disk ( $R \approx 7$ AU; e.g., Pontoppidan et al. 2008, Pérez et al. 2014), which is relatively further away from the central star compared to other transitional disks. Recent ALMA observations reveal the existence of a bright asymmetry in the south of the dust disk (Pérez et al. 2014). The $H$-band scattered light emission shows smooth distribution extending to $\sim 80 \mathrm{AU}$ without apparent asymmetry (Follette et al. 2013), which differs from that of submm imaging (e.g., Pérez et al. 2014). This indicates the different spatial distributions of small and large (mm-sized) grains, which could result from a local gas-density enhancement (e.g., via disk vortices). Geers et al. (2006) pointed out that the $11.2 \mu$ m feature detected in the IRS spectrum is broader and red-shifted similar to those of HD 135344B and LkH $\alpha 330$.

\section{A.59. SU Aur}

SU Aur (a.k.a. HD 282624) is probably a classical TTS (e.g., Furlan et al. 2006) with a spectral type of G1-2III (e.g., Calvet et al. 2004; Jeffers et al. 2014) and strong photometric variability (DeWarf et al. 2003). X-ray emission from SU Aur has been observed (e.g., Franciosini et al. 2007), of which the origin is most likely associated with magnetic activity rather than accretion like other young late-type stars with hot corona and flares. The inner radius of its disk is derived to be $\approx 0.14 \mathrm{AU}$ from the central star with a high inclination of $\sim 62^{\circ}$ (e.g., Akeson et al. 2002). Direct imaging of the SU Aur disk indicates an outer disk radius of $\sim 500 \mathrm{AU}$ (e.g., Chakraborty \& Ge 2004; Jeffers et al. 2014). Jeffers et al. (2014) suggested the presence of very small grains in the surface layers of the disk with strong 
turbulence indicating enhanced dust mixing in the disk. Using $H$-band observations, de Leon et al. (2015) revealed a pair of asymmetric tail structures, reaching to a few hundred AU from the disk, which might be explained by tidal interaction with an unseen brown dwarf. The presence of weak PAH emission unlike other late-type TTSs indicates that SU Aur has a flared disk (e.g., Keller et al. 2008; Jeffers et al. 2014). The PAH profiles at 7-9 $\mu \mathrm{m}$ belong to a "Class C" PAH spectrum (e.g., Peers et al. 2002). The " $7.7 \mu \mathrm{m}$ " feature peaks at $\approx 8.2 \mu \mathrm{m}$ and the $8.6 \mu \mathrm{m}$ feature is only marginal (e.g., Keller et al. 2008).

\section{A.60. T Cha}

T Cha is a nearby TTS (Sp. type: K0; e.g., Murphy et al. 2013), of which the distance was previously considered to be $\sim 66 \mathrm{pc}$ (e.g., van den Ancker et al. 1998) and is now commonly accepted to be $\sim 108$ pc (e.g., Torres et al. 2008) with the recent confirmation of its membership in the $\epsilon$ Cha association (e.g., Murphy et al. 2013). It is a wide binary (T Cha AB) with a separation of $0.2 \mathrm{pc}$ (Kastner et al. 2012). Geers et al. (2007b) detected weak 3.3 and $11.2 \mu \mathrm{m}$ features in ISAAC $L$-band and VISIR $N$-band spectra, both of which are unresolved placing an upper limit of $\lesssim 0^{\prime \prime} 34$ and $\lesssim 0^{\prime \prime} 19(\approx 37$ and $21 \mathrm{AU}$ at $108 \mathrm{pc})$ on the spatial extents, respectively. Olofsson et al. (2013) suggested that PAH molecules cannot be located at the inner edge of the outer disk $(\approx 12 \mathrm{AU})$ and might have been transported outwards by radiation pressure or stellar wind. Similar to HD 135344, without evident crystalline silicate emission, the $11.2 \mu \mathrm{m}$ feature is broad and red-shifted with respect to that of the diffuse ISM and is possibly indicative of the presence of very hot PAHs in the innermost part of the disk (Geers et al. 2006). By modeling the SED, Brown et al. (2007) inferred a disk structure with an inner radius of $0.08 \mathrm{AU}$ and an inner hole at $0.2-15 \mathrm{AU}$ (assuming a distance of $66 \mathrm{pc}$ ). Within this inner hole, a planet candidate has been found ( $R \approx 6.7 \mathrm{AU}$; Huélamo et al. 2011). Also, Olofsson et al. (2011) spatially resolved the innermost dusty disk to be extremely narrow and close to the star $(\sim 0.13-0.17 \mathrm{AU})$, and the outer dust disk extends up to $\sim 80 \mathrm{AU}$ whereas the gas disk is more extended with a radius of $\sim 230 \mathrm{AU}$ (Huélamo et al. 2015).

\section{A.61. TY CrA}

TY CrA (a.k.a. CD-37 13024) is a long-known tertiary system with an eclipsing binary (all within 1.5 AU to each other; e.g., Casey et al. 1998). Recent observations revealed the fourth star in the system, with a separation of $\sim 0$ "' 3 or 40 AU from TY CrA, making the TY CrA system quadruple (e.g., Chauvin et al. 2003). TY CrA is embedded in the reflection 
nebula NGC 6726/6727 located near the R Corona Australis star-forming region. In the IR images, TY CrA is surrounded by a nebulosity including a bar-like structure (e.g., Boersma et al. 2009). The observed $I S O$ spectrum (with a large aperture) is most likely dominated by the nebulosity (e.g., the bar) rather than TY CrA itself (e.g., Siebenmorgen et al. 2000; Geers et al. 2007b). The $3.3 \mu \mathrm{m}$ PAH emission has been spatially resolved with VLT/ISAAC ( $~ 54$ AU; Geers et al. 2007b), which confirms the presence of PAH emission in the TY CrA disk. Boersma et al. (2009) extracted spatially-resolved silicate and PAH spectra and found that the amorphous silicate emission peaking at $\sim 9.8 \mu \mathrm{m}$ is seen up to $\sim 130 \mathrm{AU}$, whereas the PAH emission is more extended up to $\sim 390 \mathrm{AU}$. In addition to the major PAH features, a prominent $11.0 \mu \mathrm{m}$ feature (e.g., Roche et al. 1991) as well as a weak feature at $\sim 12.0 \mu \mathrm{m}$ (e.g., Boersma et al. 2009) are detected. The spatial distributions of the 8.6, 11.0, and $11.2 \mu \mathrm{m}$ features show differences: the $11.0 \mu \mathrm{m}$ emission is most confined while the $11.2 \mu \mathrm{m}$ emission is most extended. This can be explained in terms of different band carriers with different ionization fraction of PAHs (i.e., $8.6 \mu \mathrm{m}$ : charged PAHs, $11.0 \mu \mathrm{m}$ : the out-of-plane bending mode in cationic PAHs, and $11.2 \mu \mathrm{m}$ : the out-of-plane bending mode in neutral PAHs; e.g., Hony et al. 2001, Bauschlicher et al. 2008).

\section{A.62. UX Tau}

UX Tau (a.k.a. HD 285846) is a pre-main sequence triple system consisting of one classical TTS (UX Tau A) and two WTTSs (UX Tau B and C: Sp. type of M2 and M5, respectively; Furlan et al. 2006). The primary, UX Tau A (Sp. type: K2-G8; e.g., Furlan et al. 2006; Wahhaj et al. 2010; Pinilla et al. 2014), is the only component which shows

evidence for a circumstellar disk. The disk is known to be pre-transitional, characterized by an inner optically thick disk ( $R \lesssim 0.21 \mathrm{AU}$; e.g., Espaillat et al. 2010) and a gap (e.g., Espaillat et al. 2007). Andrews et al. (2011) presented a spatially resolved cavity with a radius of $\sim 25 \mathrm{AU}$ at $880 \mu \mathrm{m}$, also confirmed by $3 \mathrm{~mm}(100 \mathrm{GHz})$ observations (Pinilla et al. 2014). The $H$-band polarimetric observations show a strongly polarized disk around UX Tau A which extends to $\sim 120 \mathrm{AU}$ (Tanii et al. 2012). The observed PAH features in the Spitzer/IRS spectra are likely to be dominated by UX Tau A (Furlan et al. 2006), one of the coolest TTSs $\left(T_{\text {eff }} \sim 5520 \mathrm{~K}\right)$ with detection of PAH emission. Also, the IRS spectra show a very weak $10 \mu \mathrm{m}$ silicate feature, a strong $20 \mu \mathrm{m}$ feature and some minor ones at $\gtrsim 20 \mu \mathrm{m}$ due to crystalline silicates (Furlan et al. 2006; Espaillat et al. 2010). This indicates a lack of small silicate grains, implying grain growth by collisional coagulation in the disk (e.g., Tanii et al. 2012). 


\section{A.63. V590 Mon}

V590 Mon (a.k.a. LkH $\alpha$ 25) is a HAeBe star with a spectral type of B8ep+sh (Juhász et al. 2010) or A0 (Habart et al. 2004a). The distance to this object is widely adopted to be $\sim 800$ pc (e.g., Liu et al. 2011), but a recent survey with the X-Shooter spectrograph mounted at the VLT suggests a distance of $\sim 1722_{-160}^{+171}$ pc (Fairlamb et al. 2015). Current estimates of its luminosity show large discrepancy ranging from $\sim 9 L_{\odot}$ (Habart et al. 2004a) to $\sim 295 L_{\odot}$ (Liu et al. 2011). Habart et al. (2004a) placed an upper limit of $\sim 1200 \mathrm{AU}$ for the spatial extent of the PAH emission. A tentative detection of the nanodiamond feature at $3.53 \mu \mathrm{m}$ is reported (Acke \& van den Ancker 2006).

\section{A.64. V892 Tau}

V892 Tau (a.k.a. Elias 3-1) is a HAeBe star with a spectral type of B8-A6 (e.g., Furlan et al. 2006; Alonso-Albi et al. 2009; van der Marel et al. 2016) located in the Taurus-Aurigae star-forming region and suffering severe extinction $\left(A_{V} \sim 4.05-11\right.$ mag; e.g., Keller et al. 2008; Monnier et al. 2008; Liu et al. 2011; van der Marel et al. 2016). An effective temperature of $T_{\text {eff }}=8000 \mathrm{~K}$ from Alonso-Albi et al. (2009) is adopted in this paper, but somewhat higher $T_{\text {eff }}$ (e.g., $\sim 11000 \mathrm{~K}$ ) has also been reported in the literature (e.g., Keller et al. 2008; van der Marel et al. 2016). Depending on the assumed $A_{V}$, its $L_{\star}$ ranges from $\sim 21 L_{\odot}$ up to a few hundred $L_{\odot}$ (e.g., Monnier et al. 2008; Alonso-Albi et al. 2009; Menu et al. 2015; van der Marel et al. 2016). V892 Tau is associated with a faint reflection nebula most brightly seen at $24 \mu \mathrm{m}$ (Mooley et al. 2013). High-resolution IR observations showed that V892 Tau is a close binary with a separation of $\sim 7.7 \mathrm{AU}$ and nearly equal brightness (e.g., Smith et al. 2005; Monnier et al. 2008). It also has a faint T Tauri stellar companion, separated by $\sim 44^{\prime \prime} 1$ (Smith et al. 2005). Monnier et al. (2008) have spatially resolved the V892 Tau disk in MIR showing an asymmetric circumbinary disk inclined at $\sim 60^{\circ}$ with an inner hole diameter of $35 \mathrm{AU}$. Using interferometric observations at 1.3 and $2.7 \mathrm{~mm}$, Hamidouche (2010) resolved its disk with a radius of $\sim 100 \mathrm{AU}$ and inferred grain growth in the disk (up to cm size) based on a low opacity $(\beta=1.1)$, which is also supported by submm observations (Sandell et al. 2011).

\section{A.65. VV Ser}

VV Ser is a Herbig Ae star (Sp. type: A0Vevp, Juhász et al. 2010; or B7, Cauley et al. 2016). The distance to VV Ser is unknown, ranging from $\sim 230$ to $614_{-88}^{+99} \mathrm{pc}$ (e.g., Alecian 
et al. 2013; Montesinos et al. 2009). This object shows the characteristics of UX Ori type stars (e.g., Herbst \& Shevchenko 1999) and evidence of an optically thin disk from NIR polarimetric measurements (Pereyra et al. 2009). Habart et al. (2004a) estimated the PAH emission extension to be $\approx 410 \mathrm{AU}$ at a distance of $440 \mathrm{pc}$. Spitzer images at $5.6-70 \mu \mathrm{m}$ show a very extended $\left(\gtrsim 4^{\prime}\right)$, bright nebulosity around VV Ser, which is most likely due to transiently-heated very small grains and PAH molecules (Pontoppidan et al. 2007). In addition, a wedge-shaped dark bend extending across the nebulosity appears, attributed to a large shadow cast by the small $(\sim 50 \mathrm{AU})$ central disk. This is consistent with an almost edge-on geometry of the disk around VV Ser, as revealed by NIR interferometry (e.g., Eisner et al. 2003) and NIR polarization (Pereyra et al. 2009). NIR H I emission lines with variable line profiles are detected (Garcia Lopez et al. 2016), which can be explained in terms of contributions of an extended wind such as a bipolar outflow in a complex inner disk region.

\section{A.66. VX Cas}

VX Cas is a Herbig Ae star (Sp. type: A1Ve+sh; Acke \& van den Ancker 2004) located at $d=620 \pm 60 \mathrm{pc}$ (Alecian et al. 2013) or $\sim 760 \mathrm{pc}$ (Dent et al. 2005). VX Cas is considered to have a geometrically flat disk (i.e., group II object; Meeus et al. 2001). Broad and weak [O I] $6300 \AA$ emission line is detected (Acke et al. 2005), which shows a double-peaked profile. Similar to other group II objects with the detection of the [O I] emission line (e.g., HD 101412), this emission might originate from a rotating gaseous disk within the dustsublimation radius (Acke et al. 2005). Oudmaijer et al. (2001) found a slight anti-correlation between photometry and polarization of VX Cas and classified VX Cas as a UX Ori type variable. Folsom et al. (2012) identified suspiciously weak metal lines in the optical spectrum of VX Cas, which might imply $\lambda$ Boo peculiarities.

\section{A.67. WL 16}

WL 16 is a Herbig Ae star (Sp. type: B8-A7; Geers et al. 2007b) embedded in the $\rho$ Ophiuchus molecular cloud at a distance of $d \sim 125$ pc (Ressler \& Barsony 2003). This object is well-known to have one of the most extended disks in MIR, of which the MIR emission is largely dominated by PAH features (e.g., Moor et al. 1998; Ressler \& Barsony 2003). Ressler \& Barsony (2003) obtained diffraction-limited images of the WL 16 disk from 7.9 to $24.5 \mu \mathrm{m}$ and revealed that the disk size is $\sim 900 \mathrm{AU}$ in diameter. Also, they confirmed the existence of ionized PAHs in the central regions and reported the discovery of a population of larger ( $\geq 50-80 \mathrm{C}$ atom) or more hydrogenated PAHs in the periphery of the disk. Recently, Zhang 
et al. (2016) presented MIR $(8-25 \mu \mathrm{m})$ polarimetric images and spectra of WL 16 obtained with the CanariCam at the Gran Telescopio Canarias. A polarization fraction of $\sim 2 \%$ is detected, which mainly arises from aligned elongated dust grains in the foreground.

\section{A.68. Wray 15-1484}

Wray 15-1484 (a.k.a. Hen 3-1191) has a spectral type of B0:[e] (Acke \& van den Ancker 2004), and its nature is rather uncertain. It was classified as a young compact proto-planetary nebula (Le Bertre et al. 1989), but later it was considered to be a Herbig Be star (e.g., De Winter et al. 1994). Its distance was estimated to be $\approx 750 \mathrm{pc}$ (Acke \& van den Ancker 2004) although it is not well constrained (Lachaume et al. 2007). Wray 15-1484 shows a bipolar structure in $R$-band (Le Bertre et al. 1989), and an axis of NIR polarization is nearly parallel to the bipolar axis (Pereyra et al. 2009), implying scattering in an optically thin disk. Lachaume et al. (2007) performed $N$-band $(8-13 \mu \mathrm{m})$ interferometric observations with VLTI/MIDI and found that the Wray 15-1484 system can be explained in terms of a disk with an unusually high mass accretion (or excretion), a large central gap, and a binary made of two IR sources.

\section{A.69. WW Vul}

WW Vul (a.k.a HD 344361) is a Herbig Ae star (Sp. type: A2 IVe; Juhász et al. 2010 ) located at $d=700_{-150}^{+260} \mathrm{pc}$ (Alecian et al. 2013) or $d \approx 440-550 \mathrm{pc}$ (Alonso-Albi et al. 2009; Juhász et al. 2010). The extended PAH emission is reported with a radius of $\sim 88 \mathrm{AU}$ adopting a distance of $700 \mathrm{pc}$ (Habart et al. 2004a). WW Vul is considered as a UX Ori type star (e.g., Grinin et al. 1996), which is characterized by high-amplitude variability in its light curve, Algol-like minima, an increase of polarization when its brightness decreases and so on (e.g., Mora et al. 2004). WW Vul exhibits " $\beta$ Pic phenomenon" like BF Ori (e.g., Grinin et al. 1996). It shows transient absorption features in metallic lines without counterparts in hydrogen lines, suggesting the presence of circumstellar gas clouds with enhanced metallicity around WW Vul (Mora et al. 2004). Pereyra et al. (2009) found a polarization reversal between optical and NIR data for WW Vul. 


\section{REFERENCES}

Acke, B., \& van den Ancker, M. E. 2004, A\&A, 426, 151

Acke, B., \& Waelkens, C. 2004, A\&A, 427, 1009

Acke, B., van den Ancker, M. E., \& Dullemond, C. P. 2005, A\&A, 436, 209

Acke, B., \& van den Ancker, M. E. 2006, A\&A, 457, 171

Acke, B., Bouwman, J., Juhász, A., et al. 2010, ApJ, 718, 558

Akeson, R. L., Ciardi, D. R., van Belle, G. T., \& Creech-Eakman, M. J. 2002, ApJ, 566, 1124

Alecian, E., Catala, C., Wade, G. A., et al. 2008, MNRAS, 385, 391

Alecian, E., Wade, G. A., Catala, C., et al. 2013, MNRAS, 429, 1001

Alencar, S. H. P., Melo, C. H. F., Dullemond, C. P., et al. 2003, A\&A, 409, 1037

Allamandola, L. J., Tielens, A. G. G. M., \& Barker, J.R. 1985, ApJ, 290, L25

Alonso-Albi, T., Fuente, A., Bachiller, R., et al. 2009, A\&A, 497, 117

Andersson, M. P., Blomquist, J., \& Uvdal, P. 2005, J. Chem. Phys., 123, 224714

Andrews, S. M., Wilner, D. J., Espaillat, C., et al. 2011, ApJ, 732, 42

Augereau, J. C., Lagrange, A. M., Mouillet, D., \& Ménard, F. 1999, A\&A, 350, L51

Augereau, J. C., Lagrange, A. M., Mouillet, D., \& Ménard, F. 2001, A\&A, 365, 78

Augereau, J. C., \& Papaloizou, J. C. B. 2004, A\&A, 414, 1153

Baines, D., Oudmaijer, R. D., Porter, J. M., \& Pozzo, M. 2006, MNRAS, 367, 737

Bakes, E. L. O., \& Tielens, A. G. G. M. 1994, ApJ, 427, 822

Barker, J.R., Allamandola, L.J., \& Tielens, A.G.G.M. 1987, ApJ, 315, L61

Bary, J. S., Weintraub, D. A., Shukla, S. J., Leisenring, J. M., \& Kastner, J. H. 2008, ApJ, 678, 1088-1098

Bauschlicher, C. W., Jr., Peeters, E., \& Allamandola, L. J. 2008, ApJ, 678, 316-327

Bauschlicher, C. W., Jr., Boersma, C., Ricca, A., et al. 2010, ApJS, 189, 341-351 
Benisty, M., Juhasz, A., Boccaletti, A., et al. 2015, A\&A, 578, L6

Benisty, M., Perraut, K., Mourard, D., et al. 2013, A\&A, 555, A113

Bernstein, M.P., Sandford, S.A., \& Allamandola, L.J. 1996, ApJ, 472, L127

Biller, B. A.,, Lacour, S., Juhász, A., et al. 2012, ApJ, 753, L38

Biller, B. A., Males, J., Rodigas, T., et al. 2014, ApJL, 792, L22

Biller, B. A., Liu, M. C., Rice, K., et al. 2015, MNRAS, 450, 4446

Blondel, P. F. C., \& Djie, H. R. E. T. A. 2006, A\&A, 456, 1045

Boersma, C., Bouwman, J., Lahuis, F., et al. 2008, A\&A, 484, 241

Boersma, C., Peeters, E., Martín-Hernández, N. L., et al. 2009, A\&A, 502, 175

Boissier, J., Alonso-Albi, T., Fuente, A., et al. 2011, A\&A, 531, A50

Borges Fernandes, M., Kraus, M., Lorenz Martins, S., \& de Araújo, F. X. 2007, MNRAS, 377,1343

Borowski, P. 2012, J. Phys. Chem. A, 116, 3866

Bouret, J.-C., Martin, C., Deleuil, M., Simon, T., \& Catala, C. 2003, A\&A, 410, 175

Bouwman, J., Meeus, G., de Koter, A., et al. 2001, A\&A, 375, 950

Brooke, T. Y., Tokunaga, A. T., \& Strom, S. E. 1993, AJ, 106, 656

Brown, J. M., Blake, G. A., Dullemond, C. P., et al. 2007, ApJ, 664, L107

Brown, J. M., Blake, G. A., Qi, C., Dullemond, C. P., \& Wilner, D. J. 2008, ApJ, 675, L109

Brown, J. M., Blake, G. A., Qi, C., et al. 2009, ApJ, 704, 496

Brown, J. M., Herczeg, G. J., Pontoppidan, K. M., \& van Dishoeck, E. F. 2012a, ApJ, 744, 116

Brown, J. M., Rosenfeld, K. A., Andrews, S. M., Wilner, D. J., \& van Dishoeck, E. F. 2012b, ApJ, 758, L30

Calvet, N., Muzerolle, J., Briceño, C., et al. 2004, AJ, 128, 1294

Caratti o Garatti, A., Tambovtseva, L. V., Garcia Lopez, R., et al. 2015, A\&A, 582, A44 
Carmona, A., van den Ancker, M. E., Audard, M., et al. 2010, A\&A, 517, A67

Carmona, A., van der Plas, G., van den Ancker, M. E., et al. 2011, A\&A, 533, A39

Casassus, S., van der Plas, G., M, S. P., et al. 2013, Nature, 493, 191

Casassus, S., Wright, C. M., Marino, S., et al. 2015, ApJ, 812, 126

Casey, B. W., Mathieu, R. D., Vaz, L. P. R., Andersen, J., \& Suntzeff, N. B. 1998, AJ, 115, 1617

Catala, C., Czarny, J., Felenbok, P., Talavera, A., \& The, P. S. 1991, A\&A, 244, 166

Cauley, P. W., \& Johns-Krull, C. M. 2016, ApJ, 825, 147

Chakraborty, A., \& Ge, J. 2004, AJ, 127, 2898

Chapillon, E., Guilloteau, S., Dutrey, A., \& Piétu, V. 2008, A\&A, 488, 565

Chauvin, G., Lagrange, A.-M., Beust, H., et al. 2003, A\&A, 406, L51

Chen, C.H., Sargent, B.A., Bohac, C., et al. 2006, ApJS, 166, 351

Chiang, E., \& Youdin, A. N. 2010, Annual Review of Earth and Planetary Sciences, 38, 493

Chiar, J. E., Tielens, A. G. G. M., Whittet, D. C. B., et al. 2000, ApJ, 537, 749

Collins, K. A., Grady, C. A., Hamaguchi, K., et al. 2009, ApJ, 697, 557

Connelley, M. S., Reipurth, B., \& Tokunaga, A. T. 2008, AJ, 135, 2496

Coulson, I. M., \& Walther, D. M. 1995, MNRAS, 274, 977

Currie, T., Cloutier, R., Brittain, S., et al. 2015, ApJ, 814, L27

Currie, T., Grady, C. A., Cloutier, R., et al. 2016, ApJ, 819, L26

de Leon, J., Takami, M., Karr, J. L., et al. 2015, ApJ, 806, L10

Dent, W. R. F., Greaves, J. S., \& Coulson, I. M. 2005, MNRAS, 359, 663

Devine, D., Grady, C. A., Kimble, R. A., et al. 2000, ApJ, 542, L115

DeWarf, L. E., Sepinsky, J. F., Guinan, E. F., Ribas, I., \& Nadalin, I. 2003, ApJ, 590, 357

de Winter, D., Grady, C. A., van den Ancker, M. E., Pérez, M. R., \& Eiroa, C. 1999, A\&A, 343,137 
de Winter, D., The, P. S., \& Perez, M. R. 1994, The Nature and Evolutionary Status of Herbig Ae/Be Stars, 62, 413

Donehew, B., \& Brittain, S. 2011, AJ, 141, 46

Dong, R., Zhu, Z., Rafikov, R. R., \& Stone, J. M. 2015, ApJ, 809, L5

Draine, B.T., \& Li, A. 2001, ApJ, 551, 807

Draine, B.T., \& Li, A. 2007, ApJ, 657, 810

Dunkin, S. K., Barlow, M. J., \& Ryan, S. G. 1997, MNRAS, 290, 165

Dunkin, S. K., \& Crawford, I. A. 1998, MNRAS, 298, 275

Eisner, J. A., Lane, B. F., Akeson, R. L., Hillenbrand, L. A., \& Sargent, A. I. 2003, ApJ, 588,360

Eisner, J. A., Graham, J. R., Akeson, R. L., \& Najita, J. 2009, ApJ, 692, 309

Espaillat, C., Calvet, N., D’Alessio, P., et al. 2007, ApJ, 670, L135

Espaillat, C., D’Alessio, P., Hernández, J., et al. 2010, ApJ, 717, 441

Evans, N. J., II, Dunham, M. M., Jørgensen, J. K., et al. 2009, ApJS, 181, 321-350

Fairlamb, J. R., Oudmaijer, R. D., Mendigutía, I., Ilee, J. D., \& van den Ancker, M. E. 2015, MNRAS, 453, 976

Fedele, D., van den Ancker, M. E., Acke, B., et al. 2008, A\&A, 491, 809

Follette, K. B., Tamura, M., Hashimoto, J., et al. 2013, ApJ, 767, 10

Follette, K. B., Grady, C. A., Swearingen, J. R., et al. 2015, ApJ, 798, 132

Folsom, C. P., Wade, G. A., Kochukhov, O., et al. 2008, MNRAS, 391, 901

Folsom, C. P., Bagnulo, S., Wade, G. A., et al. 2012, MNRAS, 422, 2072

Franciosini, E., Scelsi, L., Pallavicini, R., \& Audard, M. 2007, A\&A, 471, 951

Friedemann, C., Guertler, J., \& Loewe, M. 1996, A\&AS, 117, 205

Fuente, A., Martin-Pintado, J., Rodriguez-Franco, A., \& Moriarty-Schieven, G. D. 1998, A\&A, 339, 575 
Fujiwara, H., Honda, M., Kataza, H., et al. 2006, ApJ, 644, L133

Fukagawa, M., Hayashi, M., Tamura, M., et al. 2004, ApJ, 605, L53

Fukagawa, M., Tamura, M., Itoh, Y., et al. 2010, PASJ, 62, 347

Fukagawa, M., Tsukagoshi, T., Momose, M., et al. 2013, PASJ, 65, L14

Furlan, E., Hartmann, L., Calvet, N., et al. 2006, ApJS, 165, 568

Garcia Lopez, R., Kurosawa, R., Caratti o Garatti, A., et al. 2016, MNRAS, 456, 156

Garufi, A., Quanz, S. P., Schmid, H. M., et al. 2014, A\&A, 568, A40

Geers, V. C., Augereau, J.-C., Pontoppidan, K. M., et al. 2006, A\&A, 459, 545

Geers, V. C., Pontoppidan, K. M., van Dishoeck, E. F., et al. 2007a, A\&A, 469, L35

Geers, V. C., van Dishoeck, E. F., Visser, R., et al. 2007b, A\&A, 476, 279

Ginski, C., Stolker, T., Pinilla, P., et al. 2016, A\&A, 595, A112

Gómez de Castro, A. I., Loyd, R. O. P., France, K., Sytov, A., \& Bisikalo, D. 2016, ApJ, 818, L17

Grady, C. A., Devine, D., Woodgate, B., et al. 2000, ApJ, 544, 895

Grady, C. A., Muto, T., Hashimoto, J., et al. 2013, ApJ, 762, 48

Gray, R. O., \& Corbally, C. J. 1998, AJ, 116, 2530

Grinin, V. P., Kiselev, N. N., Chernova, G. P., Minikulov, N. K., \& Voshchinnikov, N. V. 1991, Ap\&SS, 186, 283

Grinin, V. P., Kozlova, O. V., The, P. S., \& Rostopchina, A. N. 1996, A\&A, 309, 474

Guidi, G., Tazzari, M., Testi, L., et al. 2016, A\&A, 588, A112

Guimarães, M. M., Alencar, S. H. P., Corradi, W. J. B., \& Vieira, S. L. A. 2006, A\&A, 457, 581

Habart, E., Natta, A., \& Krügel, E. 2004a, A\&A, 427, 179

Habart, E., Boulanger, F., Verstraete, L., Walmsley, C. M., \& Pineau des Forêts, G. 2004b, A\&A, 414, 531 
Habart, E., Testi, L., Natta, A., \& Carbillet, M. 2004c, ApJ, 614, L129

Habart, E., Natta, A., Testi, L., \& Carbillet, M. 2006, A\&A, 449, 1067

Haisch, K. E., Jr., Barsony, M., Ressler, M. E., \& Greene, T. P. 2006, AJ, 132, 2675

Hales, A. S., Gledhill, T. M., Barlow, M. J., \& Lowe, K. T. E. 2006, MNRAS, 365, 1348

Hales, A. S., De Gregorio-Monsalvo, I., Montesinos, B., et al. 2014, AJ, 148, 47

Hamidouche, M., Looney, L. W., \& Mundy, L. G. 2006, ApJ, 651, 321

Hamidouche, M. 2010, ApJ, 722, 204

Harvey, P. M., Wilking, B. A., \& Joy, M. 1984, ApJ, 278, 156

Hashimoto, J., Tamura, M., Muto, T., et al. 2011, ApJ, 729, L17

Hein Bertelsen, R. P., Kamp, I., van der Plas, G., et al. 2016, A\&A, 590, A98

Herbig, G. H. 1998, ApJ, 497, 736

Herbst, W., \& Shevchenko, V. S. 1999, AJ, 118, 1043

Hillenbrand, L. A., Strom, S. E., Vrba, F. J., \& Keene, J. 1992, ApJ, 397, 613

Honda, M., Maaskant, K., Okamoto, Y. K., et al. 2012, ApJ, 752, 143

Hony, S., Van Kerckhoven, C., Peeters, E., et al. 2001, A\&A, 370, 1030

Hornbeck, J. B., Grady, C. A., Perrin, M. D., et al. 2012, ApJ, 744, 54

Hu, J. Y., The, P. S., \& de Winter, D. 1989, A\&A, 208, 213

Hubrig, S., Stelzer, B., Schöller, M., et al. 2009, A\&A, 502, 283

Hudgins, D. M., \& Allamandola, L. J. 1999, ApJ, 516, L41

Hudgins, D. M., Bauschlicher, C. W., Allamandola, L. J., \& Fetzer, J. C. 2000, J. Phys. Chem., 104, 3655

Huélamo, N., Lacour, S., Tuthill, P., et al. 2011, A\&A, 528, L7

Huélamo, N., de Gregorio-Monsalvo, I., Macias, E., et al. 2015, A\&A, 575, L5

Ilee, J. D., Fairlamb, J., Oudmaijer, R. D., et al. 2014, MNRAS, 445, 3723 
Isella, A., Tatulli, E., Natta, A., \& Testi, L. 2008, A\&A, 483, L13

Isella, A., Natta, A., Wilner, D., Carpenter, J. M., \& Testi, L. 2010, ApJ, 725, 1735

Isella, A., Pérez, L. M., Carpenter, J. M., et al. 2013, ApJ, 775, 30

Jeffers, S. V., Min, M., Canovas, H., Rodenhuis, M., \& Keller, C. U. 2014, A\&A, 561, A23

Jensen, E. L. N., Cohen, D. H., \& Gagné, M. 2009, ApJ, 703, 252

Joblin, C., Tielens, A.G.G.M., Allamandola, L.J., \& Geballe, T.R. 1996, ApJ, 458, 610

Jonkheid, B., Faas, F. G. A., van Zadelhoff, G.-J., \& van Dishoeck, E. F. 2004, A\&A, 428, 511

Juhász, A., Bouwman, J., Henning, T., et al. 2010, ApJ, 721, 431

Kama, M., Folsom, C. P., \& Pinilla, P. 2015, A\&A, 582, L10

Kamp, I., \& Dullemond, C. P. 2004, ApJ, 615, 991

Kastner, J. H., Thompson, E. A., Montez, R., et al. 2012, ApJ, 747, L23

Keller, L. D., Sloan, G. C., Forrest, W. J., et al. 2008, ApJ, 684, 411

Kessler-Silacci, J., Augereau, J.-C., Dullemond, C. P., et al. 2006, ApJ, 639, 275

Khalafinejad, S., Maaskant, K. M., Mariñas, N., \& Tielens, A. G. G. M. 2016, A\&A, 587, A62

Konishi, M., Grady, C. A., Schneider, G., et al. 2016, ApJ, 818, L23

Kóspál, Á., Ábrahám, P., Acosta-Pulido, J. A., et al. 2012, ApJS, 201, 11

Kraus, S., Hofmann, K.-H., Benisty, M., et al. 2008a, A\&A, 489, 1157

Kraus, S., Preibisch, T., \& Ohnaka, K. 2008b, ApJ, 676, 490-508

Kreplin, A., Kraus, S., Hofmann, K.-H., et al. 2012, A\&A, 537, A103

Kurosawa, R., Kreplin, A., Weigelt, G., et al. 2016, MNRAS, 457, 2236

Kurucz, R. L. 1979, ApJS, 40, 1

Lachaume, R., Preibisch, T., Driebe, T., \& Weigelt, G. 2007, A\&A, 469, 587

Lacour, S., Biller, B., Cheetham, A., et al. 2016, A\&A, 590, A90 
Langhoff, S. R. 1996, J. Phys. Chem., 100, 2819

Le Bertre, T., Heydari-Malayeri, M., Epchtein, N., Gouiffes, C., \& Perrier, C. 1989, A\&A, 225, 417

Léger, A., \& Puget, J. 1984, A\&A, 137, L5

Leinert, C., Richichi, A., \& Haas, M. 1997, A\&A, 318, 472

Leinert, C., Haas, M., Ábrahám, P., \& Richichi, A. 2001, A\&A, 375, 927

Leinert, C., van Boekel, R., Waters, L. B. F. M., et al. 2004, A\&A, 423, 537

Levato, H., \& Abt, H. A. 1976, PASP, 88, 712

Li, A. 2004, in Astrophysics of Dust (ASP Conf. Ser. 309), ed. A. N. Witt, G. C. Clayton, \& B. T. Draine (San Francisco, CA: ASP), 417

Li, A. 2009, in Small Bodies in Planetary Sciences (Lecture Notes in Physics vol. 758), ed. I. Mann, A. Nakamura, \& T. Mukai, Springer, Chapter 6, 167

Li, A., \& Draine, B.T. 2001a, ApJ, 550, 213

Li, A., \& Draine, B.T. 2001b, ApJ, 554, 778

Li, A., \& Draine, B.T. 2002, ApJ, 572, 232

Li, A., \& Draine, B.T. 2012, ApJ, 760, L35

Li, A., \& Lunine, J. I. 2003, ApJ, 594, 987

Li, D., Mariñas, N., \& Telesco, C. M. 2014, ApJ, 796, 74

Lisse, C. M., Kraemer, K. E., Nuth, J. A., Li, A., \& Joswiak, D. 2007, Icarus, 187, 69

Liu, T., Zhang, H., Wu, Y., Qin, S.-L., \& Miller, M. 2011, ApJ, 734, 22

Maaskant, K. M., Bik, A., Waters, L. B. F. M., et al. 2011, A\&A, 531, A27

Maaskant, K. M., Honda, M., Waters, L. B. F. M., et al. 2013, A\&A, 555, A64

Maaskant, K. M., Min, M., Waters, L. B. F. M., \& Tielens, A. G. G. M. 2014, A\&A, 563, A78

Maaskant, K. M., de Vries, B. L., Min, M., et al. 2015, A\&A, 574, A140 
Magazzu, A., Martin, E. L., \& Rebolo, R. 1991, A\&A, 249, 149

Malbet, F., Benisty, M., de Wit, W.-J., et al. 2007, A\&A, 464, 43

Malfait, K., Bogaert, E., \& Waelkens, C. 1998, A\&A, 331, 211

Mann, I., Köhler, M., Kimura, H., Cechowski, A., \& Minato, T. 2006, A\&A Rev., 13, 159

Mann, I., Murad, E., \& Czechowski, A. 2007, Planet. Space Sci., 55, 1000

Manoj, P., Maheswar, G., \& Bhatt, H. C. 2002, MNRAS, 334, 419

Manoj, P., Bhatt, H. C., Maheswar, G., \& Muneer, S. 2006, ApJ, 653, 657

Mariñas, N., Telesco, C. M., Fisher, R. S., \& Packham, C. 2011, ApJ, 737, 57

Marino, S., Casassus, S., Perez, S., et al. 2015, ApJ, 813, 76

Mathis, J. S., Mezger, P. G., \& Panagia, N. 1983, A\&A, 128, 212

Matter, A., Labadie, L., Kreplin, A., et al. 2014, A\&A, 561, A26

Matter, A., Labadie, L., Augereau, J. C., et al. 2016, A\&A, 586, A11

Mattioda, A. L., Allamandola, L. J., \& Hudgins, D. M. 2005, ApJ, 629, 1183

Meeus, G., Waters, L. B. F. M., Bouwman, J., et al. 2001, A\&A, 365, 476

Menu, J., van Boekel, R., Henning, T., et al. 2015, A\&A, 581, A107

Merín, B., Montesinos, B., Eiroa, C., et al. 2004, A\&A, 419, 301

Merín, B., Brown, J. M., Oliveira, I., et al. 2010, ApJ, 718, 1200-1223

Merrick, J.P., Moran, D., \& Radom, L. 2007, J. Phys. Chem. A, 111, 11683

Miroshnichenko, A. S., Levato, H., Bjorkman, K. S., \& Grosso, M. 2001, A\&A, 371, 600

Momose, M., Morita, A., Fukagawa, M., et al. 2015, PASJ, 233

Monnier, J. D., Millan-Gabet, R., Billmeier, R., et al. 2005, ApJ, 624, 832

Monnier, J. D., Tannirkulam, A., Tuthill, P. G., et al. 2008, ApJ, 681, L97

Montesinos, B., Eiroa, C., Mora, A., \& Merín, B. 2009, A\&A, 495, 901

Mooley, K., Hillenbrand, L., Rebull, L., Padgett, D., \& Knapp, G. 2013, ApJ, 771, 110 
Moore, T. J. T., Emerson, J. P., Skinner, C. J., et al. 1998, MNRAS, 299, 1209

Mora, A., Eiroa, C., Natta, A., et al. 2004, A\&A, 419, 225

Morgan, W. W., Keenan, P. C., \& Kellman, E. (ed.) 1943, in An Atlas of Stellar Spectra, with an Outline of Spectral Classification (Chicago, IL: Univ. Chicago Press)

Mouillet, D., Lagrange, A. M., Augereau, J. C., \& Ménard, F. 2001, A\&A, 372, L61

Murphy, S. J., Lawson, W. A., \& Bessell, M. S. 2013, MNRAS, 435, 1325

Muto, T., Grady, C. A., Hashimoto, J., et al. 2012, ApJ, 748, L22

Muto, T., Tsukagoshi, T., Momose, M., et al. 2015, PASJ, 67, 122

Natta, A., Testi, L., Neri, R., Shepherd, D. S., \& Wilner, D. J. 2004, A\&A, 416, 179

Neuhaeuser, R., Sterzik, M. F., Schmitt, J. H. M. M., Wichmann, R., \& Krautter, J. 1995, A\&A, 297, 391

Okamoto, Y. K., Kataza, H., Honda, M., et al. 2009, ApJ, 706, 665

Olofsson, J., Benisty, M., Augereau, J.-C., et al. 2011, A\&A, 528, L6

Olofsson, J., Benisty, M., Le Bouquin, J.-B., et al. 2013, A\&A, 552, A4

Osorio, M., Anglada, G., Carrasco-González, C., et al. 2014, ApJL, 791, L36

Oudmaijer, R. D., Palacios, J., Eiroa, C., et al. 2001, A\&A, 379, 564

Panic, O. 2009, Ph.D. Thesis,

Pech, C., Joblin, C., \& Boissel, P. 2002, A\&A, 388, 639

Peeters, E., Hony, S., Van Kerckhoven, C., et al. 2002, A\&A, 390, 1089

Pendleton, Y. J., \& Allamandola, L. J. 2002, ApJS, 138, 75

Pereyra, A., Girart, J. M., Magalhães, A. M., Rodrigues, C. V., \& de Araújo, F. X. 2009, A\&A, 501, 595

Pereyra, A., Rodrigues, C. V., \& Magalhães, A. M. 2012, A\&A, 538, A59

Pérez, L. M., Isella, A., Carpenter, J. M., \& Chandler, C. J. 2014, ApJ, 783, L13

Perrin, M. D., Duchêne, G., Kalas, P., \& Graham, J. R. 2006, ApJ, 645, 1272 
Persi, P., \& Tapia, M. 2003, A\&A, 406, 149

Piétu, V., Dutrey, A., \& Kahane, C. 2003, A\&A, 398, 565

Piétu, V., Guilloteau, S., \& Dutrey, A. 2005, A\&A, 443, 945

Piétu, V., Dutrey, A., \& Guilloteau, S. 2007, A\&A, 467, 163

Pinilla, P., Benisty, M., Birnstiel, T., et al. 2014, A\&A, 564, A51

Pinilla, P., Birnstiel, T., \& Walsh, C. 2015, A\&A, 580, A105

Pontoppidan, K. M., Dullemond, C. P., Blake, G. A., et al. 2007, ApJ, 656, 980

Pontoppidan, K. M., Blake, G. A., van Dishoeck, E. F., et al. 2008, ApJ, 684, 1323-1329

Prato, L., Greene, T. P., \& Simon, M. 2003, ApJ, 584, 853

Qi, C., Öberg, K. I., Andrews, S. M., et al. 2015, ApJ, 813, 128

Quanz, S. P., Birkmann, S. M., Apai, D., Wolf, S., \& Henning, T. 2012, A\&A, 538, A92

Quanz, S. P., Avenhaus, H., Buenzli, E., et al. 2013, ApJL, 766, L2

Quanz, S. P., Amara, A., Meyer, M. R., et al. 2015, ApJ, 807, 64

Reggiani, M., Quanz, S. P., Meyer, M. R., et al. 2014, ApJL, 792, L23

Ressler, M. E., \& Barsony, M. 2003, ApJ, 584, 832

Ricca, A., Bauschlicher, C. W., Jr., Boersma, C., Tielens, A. G. G. M., \& Allamandola, L. J. 2012, ApJ, 754, 75

Rigliaco, E., Pascucci, I., Duchene, G., et al. 2015, ApJ, 801, 31

Roche, P. F., Aitken, D. K., \& Smith, C. H. 1991, MNRAS, 252, 282

Rodigas, T. J., Follette, K. B., Weinberger, A., Close, L., \& Hines, D. C. 2014, ApJ, 791, L37

Rostopchina, A. N., Grinin, V. P., Okazaki, A., et al. 1997, A\&A, 327, 145

Sakon, I., Onaka, T., Okamoto, Y. K., et al. 2006, Advances in Geosciences, Volume 7: Planetary Science (PS), 7, 143

Sandell, G., Weintraub, D. A., \& Hamidouche, M. 2011, ApJ, 727, 26 
Schütz, O., Meeus, G., \& Sterzik, M. F. 2005, A\&A, 431, 165

Schütz, O., Meeus, G., Sterzik, M. F., \& Peeters, E. 2009, A\&A, 507, 261

Schegerer, A. A., Wolf, S., Hummel, C. A., Quanz, S. P., \& Richichi, A. 2009, A\&A, 502, 367

Seok, J. Y., \& Li, A. 2015, ApJ, 809, 22

Seok, J. Y., \& Li, A. 2016, ApJ, 818, 2

Sheret, I., Dent, W. R. F., \& Wyatt, M. C. 2004, MNRAS, 348, 1282

Shevchenko, V. S., Grankin, K. N., Ibragimov, M. A., Melnikov, S. Y., \& Yakubov, S. D. 1993, Ap\&SS, 202, 137

Siebenmorgen, R., Natta, A., Kruegel, E., \& Prusti, T. 1998, A\&A, 339, 134

Siebenmorgen, R., Prusti, T., Natta, A., \& Müller, T. G. 2000, A\&A, 361, 258

Simon, M., Dutrey, A., \& Guilloteau, S. 2000, ApJ, 545, 1034

Sloan, G. C., Hayward, T. L., Allamandola, L. J., et al. 1999, ApJ, 513, L65

Sloan, G. C., Kraemer, K. E., Price, S. D., \& Shipman, R. F. 2003, ApJS, 147, 379

Sloan, G. C., Keller, L. D., Forrest, W. J., et al. 2005, ApJ, 632, 956

Sloan, G. C., Jura, M., Duley, W. W., et al. 2007, ApJ, 664, 1144

Smith, J. D. T., Draine, B. T., Dale, D. A., et al. 2007, ApJ, 656, 770

Smith, K. W., Balega, Y. Y., Duschl, W. J., et al. 2005, A\&A, 431, 307

Smith, T. L., Clayton, G. C., \& Valencic, L. 2004, AJ, 128, 357

Sterzik, M. F., Melo, C. H. F., Tokovinin, A. A., \& van der Bliek, N. 2005, A\&A, 434, 671

Stolker, T., Dominik, C., Avenhaus, H., et al. 2016, A\&A, 595, A113

Sturm, B., Bouwman, J., Henning, T., et al. 2013, A\&A, 553, A5

Szczepanski, J., Wehlburg, C., \& Vala, M. 1995, Chemical Physics Letters, 232, 221

Tang, Y.-W., Guilloteau, S., Piétu, V., et al. 2012, A\&A, 547, A84

Tanii, R., Itoh, Y., Kudo, T., et al. 2012, PASJ, 64, 124 
Tielens, A. G. G. M. 2008, ARA\&A, 46, 289

Torres, C. A. O., Quast, G., de La Reza, R., Gregorio-Hetem, J., \& Lepine, J. R. D. 1995, AJ, 109, 2146

Torres, C. A. O., Quast, G. R., Melo, C. H. F., \& Sterzik, M. F. 2008, Young Nearby Loose Associations, ed. B. Reipurth, 757

Torres, G. 2004, AJ, 127, 1187

van Boekel, R., Waters, L. B. F. M., Dominik, C., et al. 2003, A\&A, 400, L21

van Boekel, R., Waters, L. B. F. M., Dominik, C., et al. 2004, A\&A, 418, 177

van Boekel, R., Min, M., Waters, L. B. F. M., et al. 2005, A\&A, 437, 189

van den Ancker, M. E., de Winter, D., \& Tjin A Djie, H. R. E. 1998, A\&A, 330, 145

van den Ancker, M. E., Bouwman, J., Wesselius, P. R., et al. 2000, A\&A, 357, 325

van der Marel, N., van Dishoeck, E. F., Bruderer, S., et al. 2013, Science, 340, 1199

van der Marel, N., Pinilla, P., Tobin, J., et al. 2015, ApJ, 810, L7

van der Marel, N., Verhaar, B. W., van Terwisga, S., et al. 2016, A\&A, 592, A126

van der Plas, G., van den Ancker, M. E., Fedele, D., et al. 2008, A\&A, 485, 487

van der Plas, G., Wright, C. M., Ménard, F., et al. 2016, arXiv:1609.02488

van Diedenhoven, B., Peeters, E., Van Kerckhoven, C., et al. 2004, ApJ, 611, 928

van Leeuwen, F. 2007, Astrophysics and Space Science Library, vol. 350, Hipparcos, the New Reduction of the Raw Data. Springer, Heidelberg

Verhoeff, A. P., Min, M., Acke, B., et al. 2010, A\&A, 516, A48

Verhoeff, A. P., Waters, L. B. F. M., van den Ancker, M. E., et al. 2012, A\&A, 538, A101

Vieira, S. L. A., Corradi, W. J. B., Alencar, S. H. P., et al. 2003, AJ, 126, 2971

Vural, J., Kraus, S., Kreplin, A., et al. 2014, A\&A, 569, A25

Wade, G. A., Drouin, D., Bagnulo, S., et al. 2005, A\&A, 442, L31 
Waelkens, C., Bogaert, E., \& Waters, L. B. F. M. 1994, The Nature and Evolutionary Status of Herbig Ae/Be Stars, 62, 405

Wahhaj, Z., Cieza, L., Koerner, D. W., et al. 2010, ApJ, 724, 835-854

Walsh, C., Juhász, A., Meeus, G., et al. 2016, ApJ, 831, 200

Wang, S., Looney, L. W., Brandner, W., \& Close, L. M. 2008, ApJ, 673, 315-330

Wassell, E. J., Grady, C. A., Woodgate, B., Kimble, R. A., \& Bruhweiler, F. C. 2006, ApJ, 650,985

Weidner, C., Kroupa, P., \& Bonnell, I. A. D. 2010, MNRAS, 401, 275

Weinberger, A. J., Becklin, E. E., Schneider, G., et al. 1999, ApJ, 525, L53

Weingartner, J. C., \& Draine, B. T. 2001, ApJS, 134, 263

Wyatt, M. C. 2005, A\&A, 440, 937

Yang, X. J., Glaser, R., Li, A., \& Zhong, J. X. 2013, ApJ, 776, 110

Yang, X. J., Glaser, R., Li, A., \& Zhong, J. X. 2016a, MNRAS, 462, 1551

Yang, X. J., Li, A., Glaser, R., \& Zhong, J. X. 2016b, ApJ, 825, 22

Zhang, H., Telesco, C. M., Pantin, E., et al. 2016, arXiv:1610.07906

Zwintz, K., Fossati, L., Ryabchikova, T., et al. 2014, Science, 345, 550 